January 2016

\title{
Experimental Heat Transfer Distributions Over An Aft Loaded Vane With A Large Leading Edge At Very High Turbulence Levels
}

Justin William Varty

\section{How does access to this work benefit you? Let us know!}

Follow this and additional works at: https://commons.und.edu/theses

\section{Recommended Citation}

Varty, Justin William, "Experimental Heat Transfer Distributions Over An Aft Loaded Vane With A Large Leading Edge At Very High Turbulence Levels" (2016). Theses and Dissertations. 2080.

https://commons.und.edu/theses/2080

This Thesis is brought to you for free and open access by the Theses, Dissertations, and Senior Projects at UND Scholarly Commons. It has been accepted for inclusion in Theses and Dissertations by an authorized administrator of UND Scholarly Commons. For more information, please contact und.commons@library.und.edu. 


\title{
EXPERIMENTAL HEAT TRANSFER DISTRIBUTIONS OVER AN AFT LOADED VANE WITH A LARGE LEADING EDGE AT VERY HIGH TURBULENCE LEVELS
}

\author{
by
}

\author{
Justin William Varty \\ Bachelor of Science, University of North Dakota, 2014 \\ A Thesis \\ Submitted to the Graduate Faculty \\ of the \\ University of North Dakota
}
In partial fulfillment of the requirements
for the degree of
Master of Science

Grand Forks, North Dakota

August

2016 
This thesis, submitted by Justin W. Varty in partial fulfillment of the requirements for the degree of Master of Science from the University of North Dakota has been read by the Faculty Advisory Committee under whom the work has been done and is hereby approved.

\section{Dr. Forrest Ames}

Date

\section{Dr. Clement Tang}

Date

Dr. Nanak Grewal

Date

This thesis is being submitted by the appointed advisory committee as having met all of the requirements of the School of Graduate Studies at the University of North Dakota and is hereby approved.

\section{Grant McGimpsey \\ Dean of the School of Graduate Studies}

\section{Date}




\section{PERMISSION}

Title EXPERIMENTAL HEAT TRANSFER DISTRIBUTIONS OVER AN AFT
LOADED VANE WITH A LARGE LEADING EDGE AT VERY HIGH
TUBULENCE LEVELS

Department Mechanical Engineering

Degree $\quad$ Master of Science

In presenting this thesis in partial fulfillment of the requirements for a graduate degree from the University of North Dakota, I agree that the library of the University shall make it freely available for inspection. I further agree that the permission for extensive copying for scholarly purposes may be granted by the professor who supervised my thesis work or, in his absence, by the Chairperson of the department or the dean of the School of Graduate Studies. It is understood that any copying or publication or other use of this thesis or pat thereof for financial gain shall not be allowed without my written permission. It is also understood that due recognition shall be given to me and to the University of North Dakota in any scholarly use which may be made of any material in my thesis.

Justin W. Varty

August 2016 


\section{TABLE OF CONTENTS}

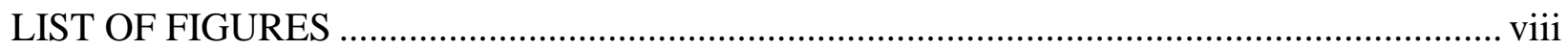

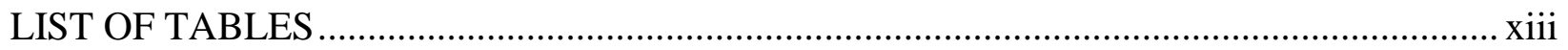

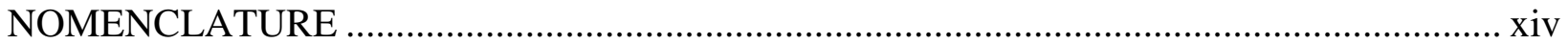

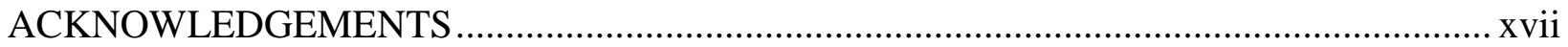

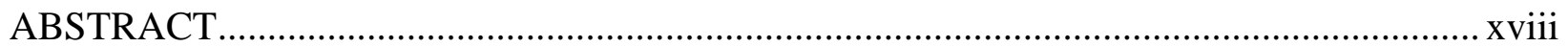

\section{CHAPTER}

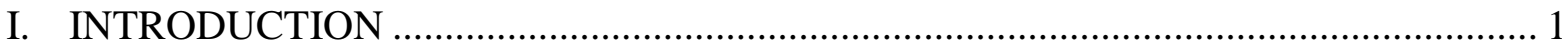

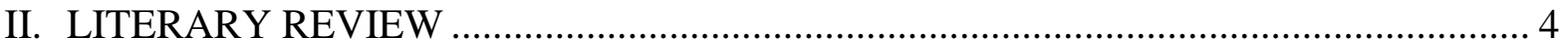

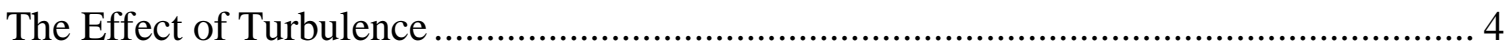

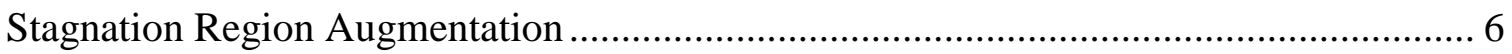

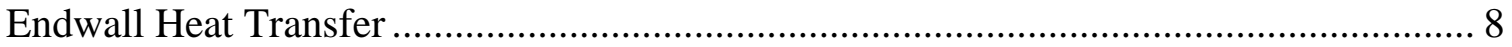

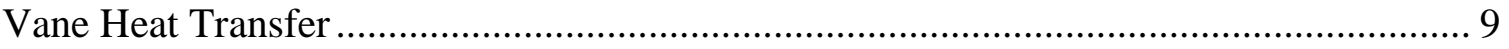

III. EXPERIMENTAL APPROACH.............................................................................. 12

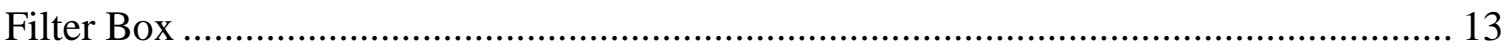

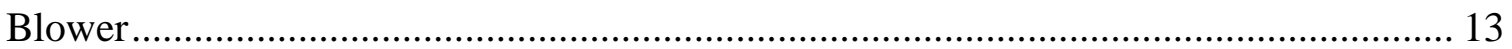

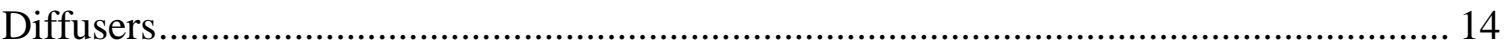

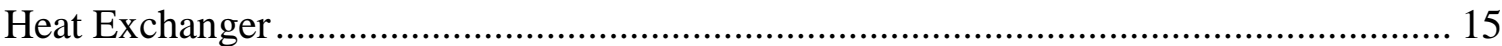


Flow Mixer.

Straightener

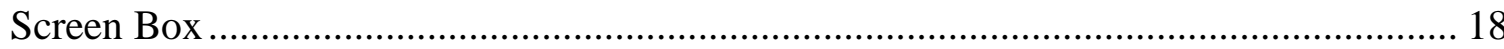

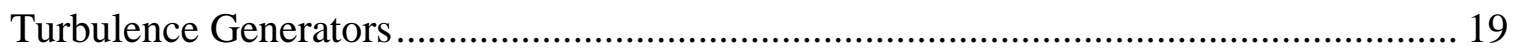

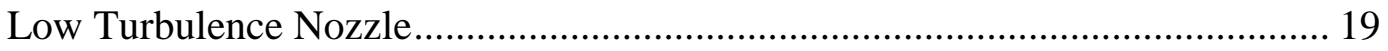

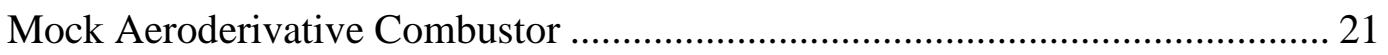

High Turbulence Nozzle ..................................................................... 22

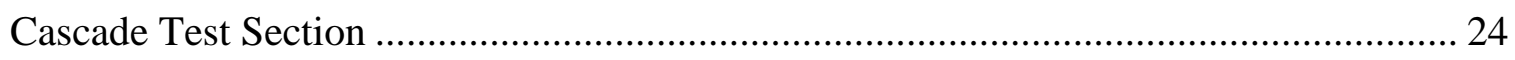

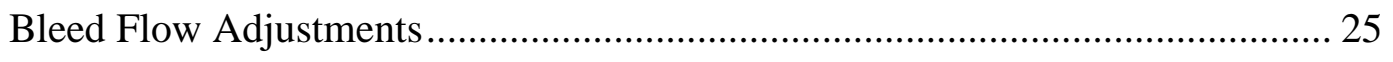

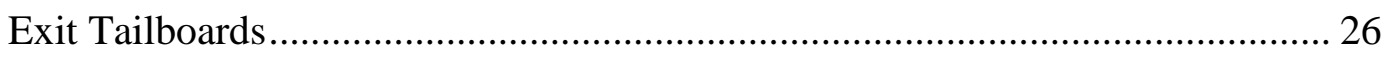

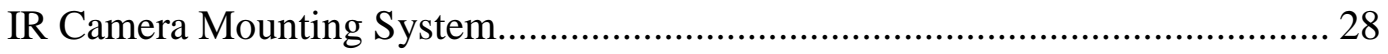

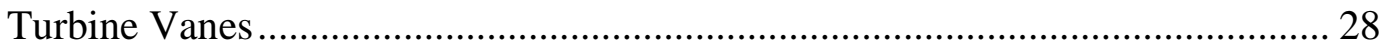

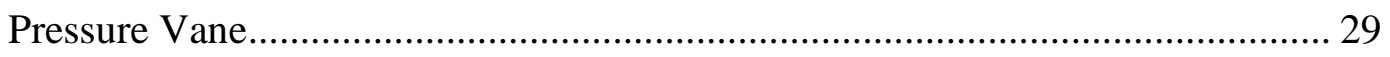

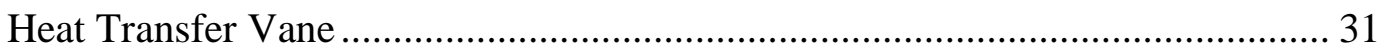

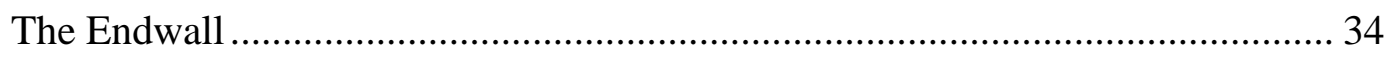

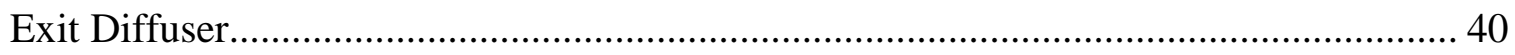

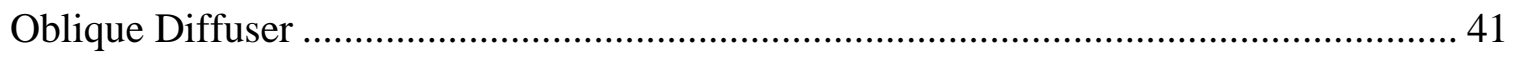

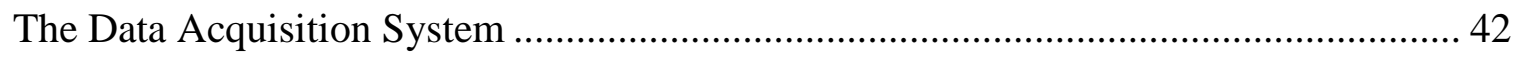

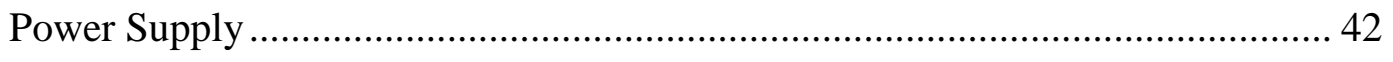

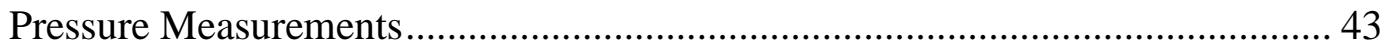




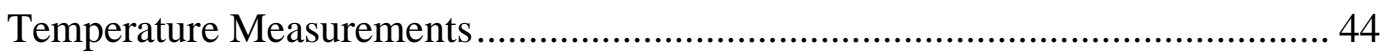

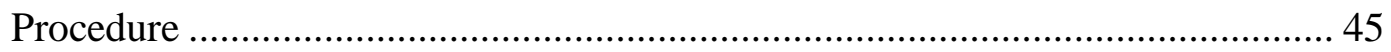

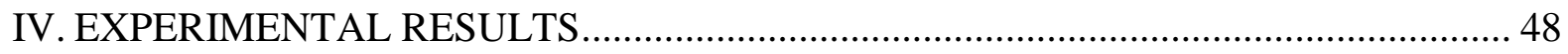

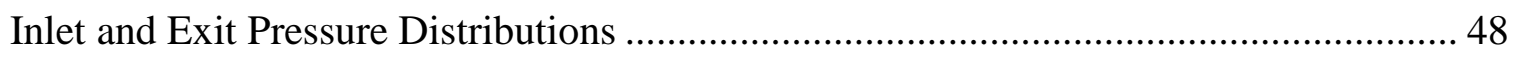

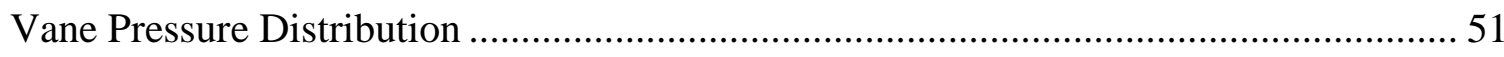

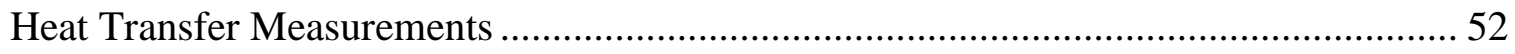

Vane Heat Transfer Distributions ............................................................................... 54

Heat Transfer Augmentation and Transition .............................................................. 57

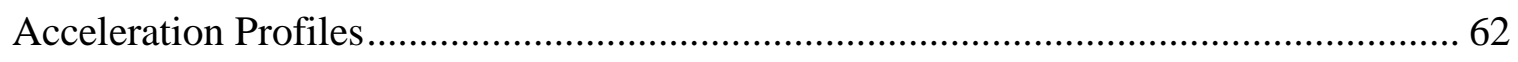

Heat Transfer Augmentation for Other Shapes .......................................................... 63

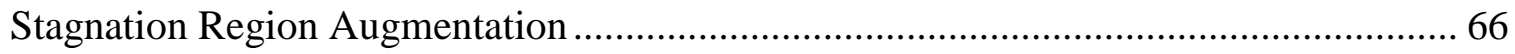

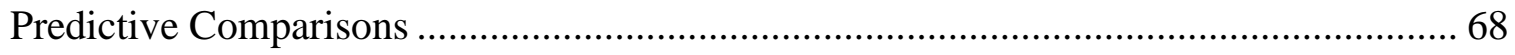

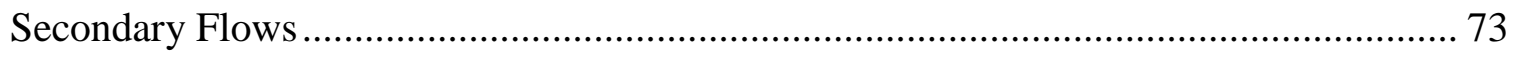

Effects of Endwall Heating on Vane Heat Transfer ...................................................... 74

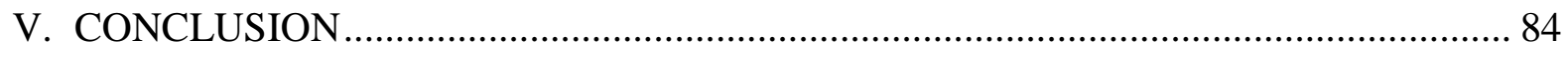

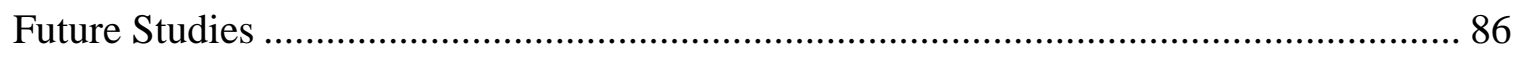

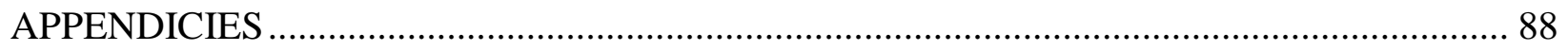

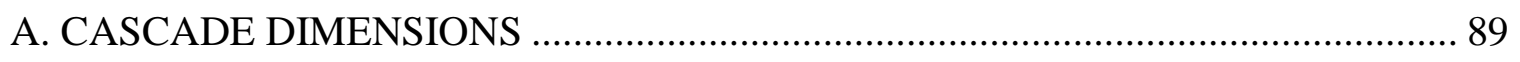

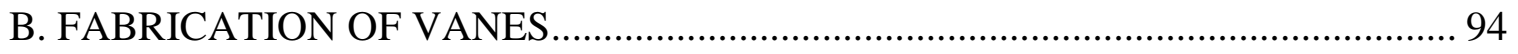

C. THE ENDWALL HEAT TRANSFER SYSTEM …………………......................... 98 
D. SUCTION SURFACE STANTON NUMBER CHARTS

E. THE EFFECTS OF ROUGHNESS ON VANE HEAT TRANSFER ...................... 110

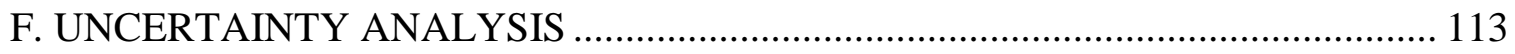

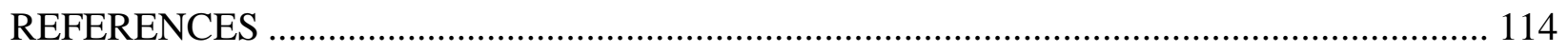




\section{LIST OF FIGURES}

Figure $\quad$ Page

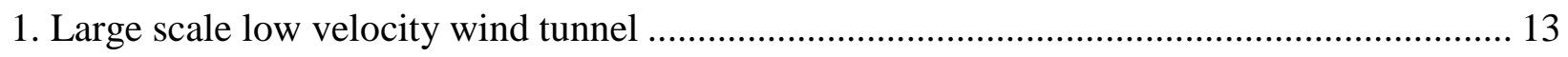

2. New York Blower used for Wind Tunnel................................................................. 14

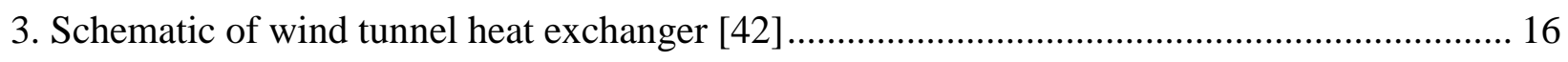

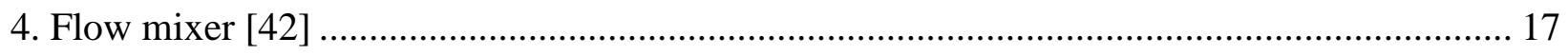

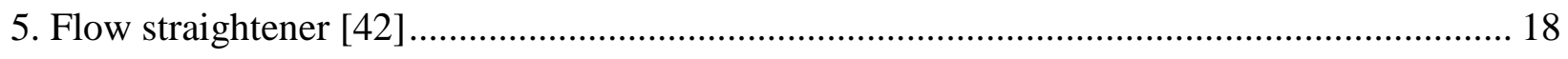

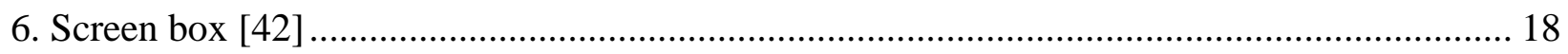

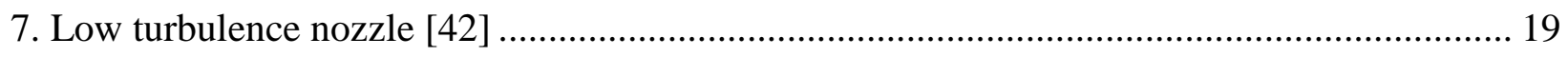

8. Small grid far configuration (left) and Small grid near configuration (right)...................... 20

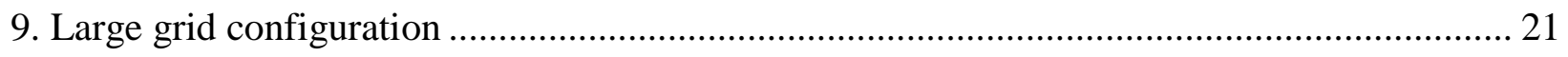

10. Exit of mock aero combustor (left) and inlet of mock aero combustor (right) [42] ............. 22

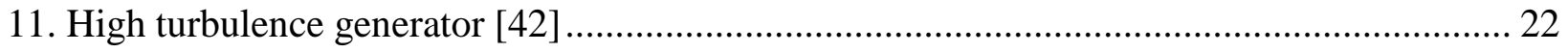

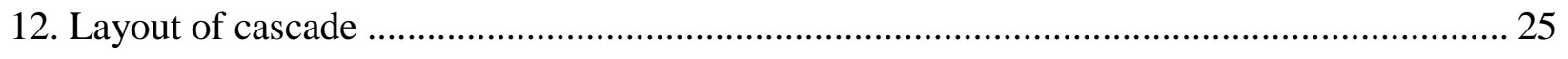

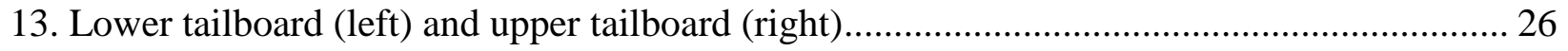

14. The tailboard with adjustable nylon rods (left) and tailboard windows (right) ................... 27

15. IR camera mount side view (left) and back view (right) …........................................ 28

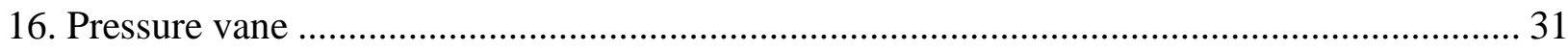

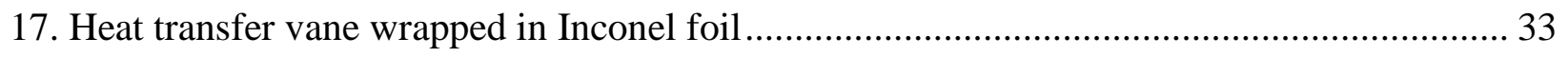

18. Painted heat transfer vane (left) painted vane with dot location grid (right) ...................... 34

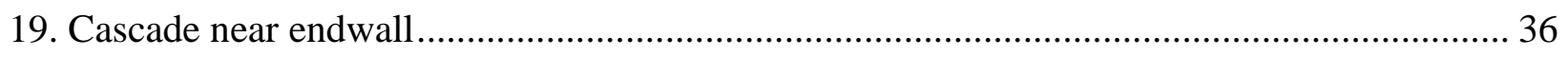


20. Vane window for instrumented vanes.

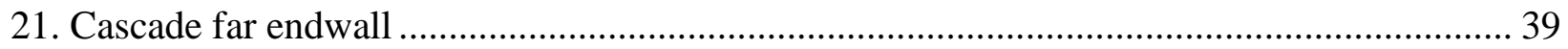

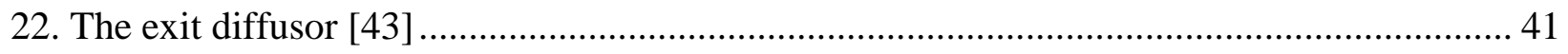

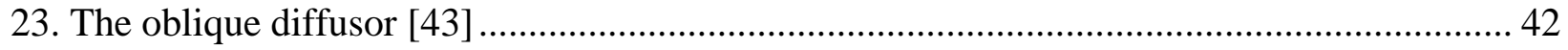

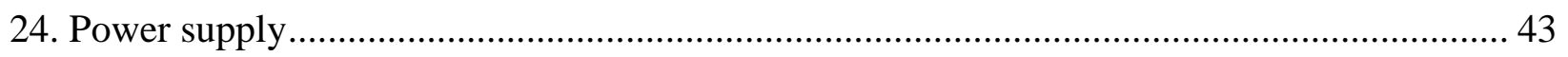

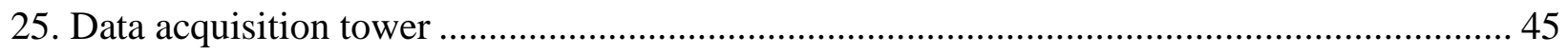

26. Low turbulence inlet pressure distribution $\operatorname{Re} 1,000,000$.............................................. 49

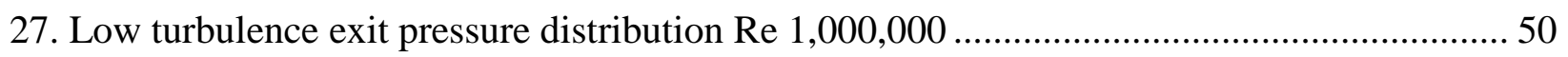

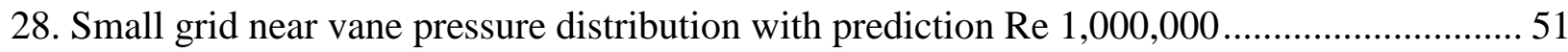

29. Vane Stanton number distributions with varying turbulence levels, $\operatorname{Re}_{\mathrm{c}}=500,000 \ldots \ldots \ldots \ldots . . .55$

30. Vane Stanton number distributions with varying turbulence levels, $\operatorname{Re}_{c}=1,000,000 \ldots \ldots \ldots .56$

31. Vane Stanton number distributions with varying turbulence levels, $\operatorname{Re}_{\mathrm{c}}=2,000,000 \ldots \ldots \ldots . .57$

32. $\mathrm{St}_{-} \mathrm{St}_{0}$ versus $\mathrm{X}$ comparisons with varying turbulence levels at $\mathrm{Re}_{\mathrm{C}}=500,000 \ldots \ldots \ldots \ldots \ldots \ldots . . .60$

33. St-St $t_{0}$ versus $X$ comparisons with varying turbulence levels at $\operatorname{Re}_{C}=1,000,000 \ldots \ldots \ldots \ldots \ldots 61$

34. St-St $t_{0}$ versus $\mathrm{X}$ comparisons with varying turbulence levels at $\mathrm{Re}_{\mathrm{C}}=2,000,000 \ldots \ldots \ldots \ldots \ldots .62$

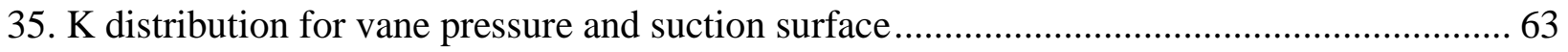

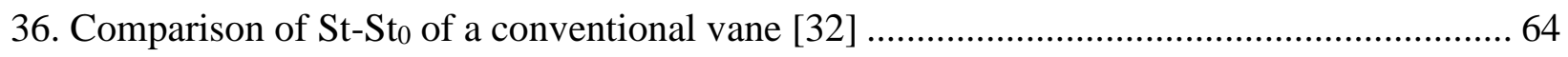

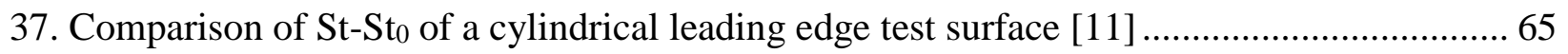

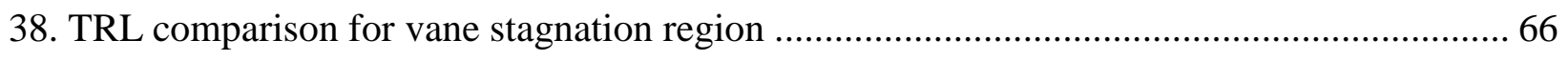

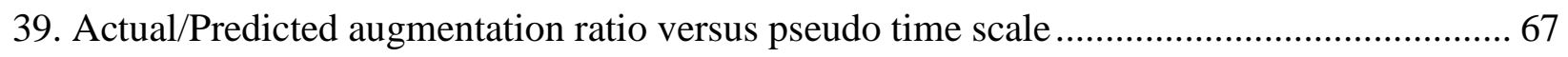

40. Predictions for vane Stanton number with varying turbulence, $\operatorname{Re}_{\mathrm{c}}=500,000 \ldots \ldots \ldots \ldots \ldots \ldots . . \ldots 9$

41. Predictions for vane Stanton number with varying turbulence, $\operatorname{Re}_{\mathrm{c}}=1,000,000 \ldots \ldots \ldots \ldots \ldots . . .71$

42. Predictions for vane Stanton number with varying turbulence, $\operatorname{Re}_{\mathrm{c}}=2,000,000 \ldots \ldots \ldots \ldots \ldots . . .72$ 
43. Langston et al. model depicting endwall vorticies.

44. Suction surface Stanton number chart $\mathrm{Re}=1,000,000, \mathrm{Tu}=0.035$, unheated starting length, bottom

45. Suction surface Stanton number chart $\mathrm{Re}=1,000,000, \mathrm{Tu}=0.035$, unheated starting length, top

46. Suction surface Stanton number chart $\mathrm{Re}=1,000,000, \mathrm{Tu}=0.081$, unheated starting length, bottom

47. Suction surface Stanton number chart $\mathrm{Re}=1,000,000, \mathrm{Tu}=0.081$, unheated starting length, top

48. Suction surface Stanton number chart $\mathrm{Re}=1,000,000, \mathrm{Tu}=0.081$, heated starting length, bottom

49. Suction surface Stanton number chart $\mathrm{Re}=1,000,000, \mathrm{Tu}=0.081$, heated starting length, top

50. Suction surface Stanton number chart $\mathrm{Re}=500,000, \mathrm{Tu}=0.081$, heated starting length, bottom 81

51. Suction surface Stanton number chart $\mathrm{Re}=500,000, \mathrm{Tu}=0.081$, heated starting length, top

52. Suction surface Stanton number chart $\mathrm{Re}=2,000,000, \mathrm{Tu}=0.081$, heated starting length, bottom

53. Suction surface Stanton number chart $\mathrm{Re}=2,000,000, \mathrm{Tu}=0.081$, heated starting length, top

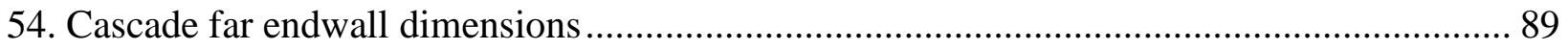

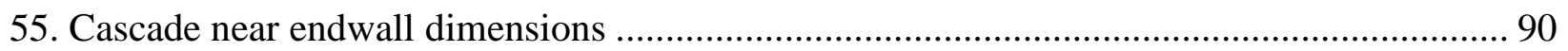

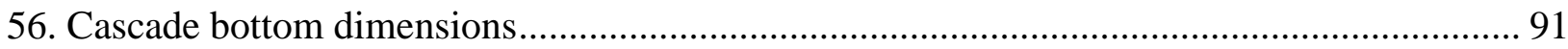

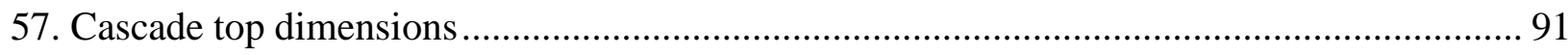

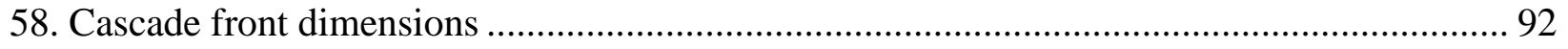

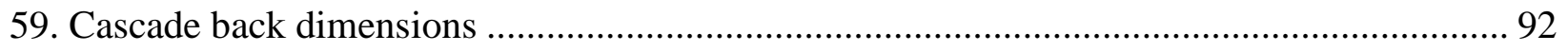

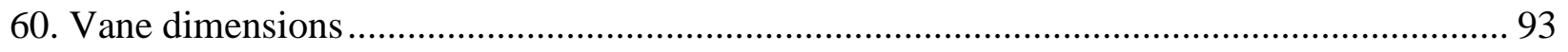


61. Endwall blower and heat exchanger

62. Endwall cooling air supply

101

63. Suction surface Stanton number chart $\mathrm{Re}=500,000, \mathrm{Tu}=0.035$, unheated starting length, bottom 102

64. Suction surface Stanton number chart $\mathrm{Re}=500,000, \mathrm{Tu}=0.035$, unheated starting length, top 103

65. Suction surface Stanton number chart $\mathrm{Re}=2,000,000, \mathrm{Tu}=0.035$, unheated starting length, bottom 104

66. Suction surface Stanton number chart $\mathrm{Re}=2,000,000, \mathrm{Tu}=0.035$, unheated starting length, top 104

67. Suction surface Stanton number chart $\mathrm{Re}=500,000, \mathrm{Tu}=0.035$, heated starting length, bottom

68. Suction surface Stanton number chart $\mathrm{Re}=500,000, \mathrm{Tu}=0.035$, heated starting length, top

69. Suction surface Stanton number chart $\mathrm{Re}=1,000,000, \mathrm{Tu}=0.035$, heated starting length, bottom 106

70. Suction surface Stanton number chart $\mathrm{Re}=1,000,000, \mathrm{Tu}=0.035$, heated starting length, top

71. Suction surface Stanton number chart $\mathrm{Re}=2,000,000, \mathrm{Tu}=0.035$, heated starting length, bottom 107

72. Suction surface Stanton number chart $\mathrm{Re}=2,000,000, \mathrm{Tu}=0.035$, heated starting length, top 107

73. Suction surface Stanton number chart $\mathrm{Re}=500,000, \mathrm{Tu}=0.081$, unheated starting length, bottom 108

74. Suction surface Stanton number chart $\mathrm{Re}=500,000, \mathrm{Tu}=0.081$, unheated starting length, top 108

75. Suction surface Stanton number chart $\mathrm{Re}=2,000,000, \mathrm{Tu}=0.081$, unheated starting length, bottom 109

76. Suction surface Stanton number chart $\mathrm{Re}=2,000,000, \mathrm{Tu}=0.081$, unheated starting length, top 109 
77. Vane heat transfer of the standard heat transfer vane vs. the painted heat transfer vane ..... 110

78. Vane heat transfer of the standard heat transfer vane vs. the painted heat transfer vane ..... 111

79. Vane heat transfer of the standard heat transfer vane vs. the painted heat transfer vane ..... 112 


\section{LIST OF TABLES}

Table Page

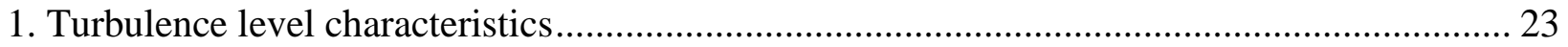

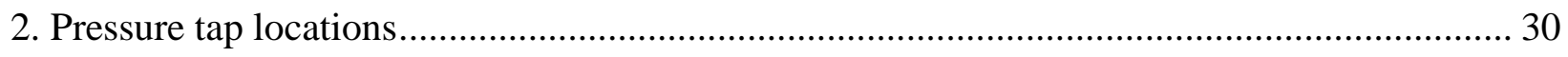

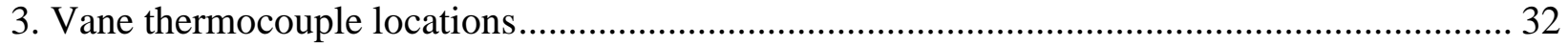

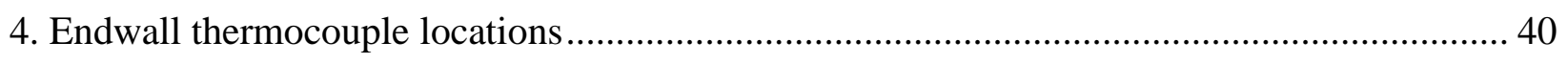


NOMENCLATURE

C true chord length, $\mathrm{m}$

$\mathrm{C}_{\mathrm{P}} \quad$ specific heat at constant pressure, $\mathrm{J} / \mathrm{kg} \cdot \mathrm{K}$

D leading edge diameter, $m$

h heat transfer coefficient, $\mathrm{W} / \mathrm{m}^{2} \cdot \mathrm{K}$

$\mathrm{h}_{0} \quad$ low turbulence heat transfer coefficient, $\mathrm{W} / \mathrm{m}^{2} \cdot \mathrm{K}$

$\mathrm{K}$ boundary layer acceleration parameter, $v / \mathrm{U}^{2} \mathrm{dU} / \mathrm{dx}$

$\mathrm{Lu}$ energy scale, $\mathrm{Lu}=1.5\left|\mathrm{u}^{\prime}\right|^{3} / \varepsilon, \mathrm{m}$

Lx longitudinal integral scale of turbulence, $\mathrm{m}$

$\mathrm{P}$ pressure, $\mathrm{Pa}$

$\mathrm{P}^{+} \quad$ Dimensionless pressure value

$\operatorname{Re}_{C}$ chord Reynolds number based on exit conditions

$\operatorname{Re}_{\mathrm{D}}$ diameter Reynolds number based on approach velocity

St Stanton number, $\mathrm{St}=\mathrm{h} / \rho \cdot \mathrm{V}_{\mathrm{EXIT}} \cdot \mathrm{Cp}$

$\mathrm{T}$ temperature, $\mathrm{K}$

$\mathrm{Tu}$ local turbulence intensity, $\mathrm{u}^{\prime} / \mathrm{U}_{\infty}$

$\mathrm{Tu}_{0}$ turbulence intensity at measurement location

$\mathrm{TRL} \mathrm{TRL}=\mathrm{Tu} \cdot \mathrm{Re}_{\mathrm{D}}{ }^{5 / 12} \cdot(\mathrm{D} / \mathrm{Lu})^{1 / 3}$

$\mathrm{U}_{\infty} \quad$ free-stream velocity, $\mathrm{m} / \mathrm{s}$

VEXIT exit velocity

Q power, W

I current, A

V voltage, $\mathrm{V}$

Q" heat flux, W/m 
A area, $\mathrm{m}^{2}$

Q" net net heat flux due to the emissivity of the foil, $\mathrm{W} / \mathrm{m}^{2}$

$\mathrm{T}_{\mathrm{h}} \quad$ heated vane temperature, $\mathrm{K}$

$\mathrm{T}_{\mathrm{a}} \quad$ adiabatic vane temperature, $\mathrm{K}$

$\mathrm{T}_{\mathrm{Th}} \quad$ total inlet temperature for heated tests, $\mathrm{K}$

$\mathrm{T}_{\mathrm{Ta}} \quad$ total intel temperature for adiabatic tests, $\mathrm{K}$

$\mathrm{T}_{\text {diff }}$ difference between heated and unheated temperature between tests, $\mathrm{K}$

u', $\left|\mathrm{u}^{\prime}\right|$ streamwise component rms fluctuation velocity, $\mathrm{m} / \mathrm{s}$

$\mathrm{V}_{\mathrm{APP}}$ approach velocity to cylinder, $\mathrm{m} / \mathrm{s}$

$\mathrm{X}$ distance, streamwise direction, $\mathrm{m}$

$\mathrm{Y}$ distance, normal to surface, $\mathrm{m}$

$\frac{d \bar{T}}{d y} \quad$ mean temperature gradient

\section{Greek Letter Symbols}

$\alpha \quad$ thermal diffusivity, $\mathrm{m}^{2} / \mathrm{s}$

$\alpha_{\mathrm{TU}}$ eddy diffusivity due to turbulence, $\mathrm{m}^{2} / \mathrm{s}$

$\alpha_{\text {TURB }}$ eddy diffusivity due to the intermittent turbulent boundary layer, $\mathrm{m}^{2} / \mathrm{s}$

$\gamma \quad$ intermittency

$\varepsilon \quad$ turbulent dissipation rate, $\mathrm{m}^{2} / \mathrm{s}^{3}$

$\varepsilon_{\mathrm{m}} \quad$ emissivity

$\lambda \quad$ Thwaite's acceleration parameter, $\lambda=\theta^{2} / \nu \mathrm{d} \mathrm{U}_{\infty} / \mathrm{dx}$

$v \quad$ kinematic viscosity, $\mathrm{m}^{2} / \mathrm{s}$

$\theta \quad$ momentum thickness, $\mathrm{m}$

$\rho \quad$ density, $\mathrm{kg} / \mathrm{m}^{3}$ 
Subscripts

0 low turbulence condition

EXIT referenced to vane exit conditions 


\section{ACKNOWLEDGEMENTS}

This project could not have been completed without the help of many people. I would like to start with the University of North Dakota Mechanical Engineering Department for providing me the opportunity to continue my education in their graduate program. I would also like to thank Dr. Ames for advising me throughout the duration of the project. I wouldn't have been able to complete this project without the knowledge and guidance he provided me over the last two years when I worked under him. This help over the years was greatly appreciated. I have to thank my advisor committee members, Dr. Clement Tang and Dr. Nanak Grewal. I am thankful towards the University Turbine System Research Program of the National Energy Technology Laboratory of the U.S. Department of Energy, and the University of North Dakota for providing the funds which allowed me to carry out this research.

There are several students who deserve my thanks as well. I would like to thank the senior design group consisting of Benjamin Stokes, Chloe Tinius, and Teagan Nelson for their work on the project. They contributed significantly in the development of the linear cascade test section used in the project. I also have to thank Loren Soma, Joseph Kingery for their help and assistance over the duration of the project. I need to thank Jay Evenstad and Gary Dubuque for all of their help and assistance with the manufacturing components of this project. 


\begin{abstract}
The purpose of this study is to experimentally investigate the effects of high free-stream turbulence on an aft loaded vane designed with a large leading edge. The large leading edge vane design was chosen to help reduce the heat transfer levels on the leading edge of the vane, while also generating a large enough area in the leading edge to allow the vane be cooled internally using a double wall cooling design. Heat transfer measurements were acquired on a linear cascade using a constant heat flux technique to help determine the effects of turbulence over this vane design. The cascade that was used was designed as a four vane, three passage linear cascade with adjustable bleed flows and tailboards which are adjustable to the periodic streamlines of the blade to blade analysis. The heat transfer measurements were taken at a wide range of Reynolds numbers, ranging from 500,000 to 2,000,000, and seven different turbulence levels. The turbulence levels range from a low turbulence condition of $0.7 \%$ to a high turbulence condition of $17.4 \%$. The other turbulence conditions are a small grid far condition $(\mathrm{Tu}=3.5 \%)$, a small grid near condition $(\mathrm{Tu}=7.9 \%)$, a large grid condition $(\mathrm{Tu}=8.0 \%)$, an aero-combustor with a decay spool condition $(\mathrm{Tu}=9.3 \%)$, and an aero-combustor closely placed to the cascade $(\mathrm{Tu}=$ 13.7\%). Inlet and exit pressure distributions along with vane pressure distributions were taken to help ensure the aerodynamic accuracy of the cascade. The heat transfer levels taken at these turbulence levels were correlated in terms of the approach flow Reynolds number and the turbulence condition. This was then compared to recent cylindrical leading edge test surface data using the TRL parameter. The surface heat transfer measurements that were taken were based off of the exit Reynolds numbers and displayed in terms of the Stanton number. These were then
\end{abstract}


compared to predictive comparisons generated from a boundary layer calculation (STAN 7), using an algebraic turbulence model (ATM), and a transition model (Mayle). At the low turbulence levels the predictions show a close resemblance to the heat transfer distributions based on the exit Stanton number. As the turbulence levels increase the predictions tend to under predict at the stagnation region of the vane and tend to predict early transition on the suction surface of the vane. The inaccuracies in the transition prediction indicate a need to account for the complex curvature effects on the suction surface. Also to help accurately predict the heat transfer the physics of the turbulent response in the stagnation region would have to be included in the modeling. Also, these inaccuracies show some of the challenges faced when using engineering turbulence models. Later, the heat transfer distributions were then compared to suction surface heat transfer data taken with a heated and unheated endwall to show the impact of secondary flows on heat transfer in this region. The heat transfer distributions taken with a heated endwall showed a dramatic increase in the heat transfer levels at the edge of the vane and should provide a more relevant representation of the heat transfer levels in this region. 


\section{CHAPTER I}

\section{INTRODUCTION}

In the recent years the competition for creating more efficient and durable gas turbine designs has pushed the industry towards new designs. However, many different parts of the country have set very restrictive emission requirements for these new designs. To meet these requirements it is necessary for a low NOx combustion system to be included in all new land based gas turbine designs. One of the drawbacks from these systems is that they tend to create very high levels of turbulence [1]. Traditionally gas turbines have used showerhead cooling designs in the leading edge regions and shaped holes on the pressure surface to meet the cooling demands on first stage vanes. Even though these designs meet the cooling demands set forth by the industry, their use of cooling air on the surface could be more efficient. Their inefficiency could be due to discharging the cooling air in low velocity areas on the vane where the turbulence can be very disruptive to downstream boundary layer growth. Also, discharging the cooling air in these regions of low velocity flow can create a higher level of mixing which can help dissipate the coolant more quickly making the film cooling less effective and heat transfer levels higher. Another problem that this system is susceptible to is having the holes in the showerhead design clog due to particulates in the air or fuel.

To overcome these drawbacks a vane with a larger leading edge could be designed allowing the leading edge to be cooled internally. Boundary layers in the stagnation region of a vane are generally laminar. The relative heat load in this area is lower compared to the areas 
further downstream. Accommodating cooling schemes internally for most of the vane is not a problem because the surface curvature is relatively flat. However, the strong surface curvature in the leading edge area can cause a problem for internal cooling. By increasing the radius of the leading edge of a vane it would not only help in decreasing the heat load for this area, but it would also create a greater area for the vane to be cooled internally. Once the air travels through the internal cooling design it is dispersed onto the surface to further cool the vane through film cooling. The enhancement of film cooling has been studied and it helpful to disperse the coolant onto the surface in areas of high acceleration [2], at least initially. More recent studies done by Buche et al. [3] and Kingery and Ames [4] shows that slot and shaped hole film cooling is significantly improved in laminar and transitional regions of flow when compared to turbulent regions. This means that to have the greatest effectiveness for the film cooling keeping the flow laminar as long as possible helps.

This study investigated a new vane design, one with a large leading edge and an aft loaded suction surface of the vane. The aft loaded design helped to delay the transition of the flow on the suction surface of the vane, thus increasing the effectiveness of the film cooling. The larger leading edge not only makes the surface curvature flatter, which helps lower the heat loads that the leading edge and more importantly the stagnation region experience, but also it should also make it easier for the leading edge to be internally cooled. This study investigated the heat transfer levels on this new vane design at seven different turbulence conditions ranging from a low turbulence condition of $0.7 \%$ to a very high turbulence condition of $17.4 \%$. Also, the tests were completed at three different Reynolds numbers. By looking at the effect of the different turbulence conditions and Reynolds numbers on the heat transfer distributions of the vane surface it was clear that there was an effect on the relative heat transfer augmentation. The 
relative augmentation in the laminar region of the vane helps provide information on the path of transition of the flow around the vane. Predictions were also made to help compare the results collected to expected levels of heat transfer. These predictions help provide a level of discussion between the two and why or how they are different.

The study also investigated the effect of secondary flows and the endwall vane interface thermal boundary condition on the suction surface heat transfer. Two different measurements were taken, one with the endwall heat on, simulating a heated starting length, and the other with the endwall heat turned off, simulating an unheated starting length. The effects of a heated endwall could be seen on the suction surface of the vane. IR camera pictures were taken on the suction surface of the vane when it was heated and unheated. This demonstrated the influence of secondary flows on the heat transfer of the vane and the effect of a heated and unheated endwall. 


\section{CHAPTER II}

\section{LITERARY REVIEW}

The testing and understanding of heat loads on turbine components in gas turbine engines are critical steps to developing reliable and efficient cooling methods for these components. First stage gas turbine vanes, which are subjected to high amounts of turbulence coming from the combustor can experience a significant rise in stagnation region heat transfer, augmentation in laminar regions of flow on the pressure and suction surfaces, and can also experience early transition due to the high turbulence levels. Even though the understanding of the flow patterns inside gas turbines has dramatically increased over the years, there are still many challenges that exist which can inhibit the accuracy of predicting the heat loads on vanes due to turbulence.

\section{The Effect of Turbulence}

The turbulence that exits from the combustor of a gas turbine can have a major role in determining the heat transfer levels on a turbine vane. One area that is especially susceptible to the changing turbulence levels is the stagnation region of the vane. The turbulence levels exiting the combustor of a turbine engine have been well documented. It has been documented that the range of turbulence levels that exit the combustor can vary from around 7\%, reported downstream from a helicopter engine [5], to up to $30 \%$ reported downstream from a DNL combustor [1]. However, the literature on turbulence measured downstream from the combustor is limited. Moss and Outfield [6] stated that to accurately predict the heat transfer on a turbine vane the knowledge of the turbulence levels exiting in the combustor is the major key. The high 
temperatures and pressures that a combustor experiences made the testing difficult for Moss and Ourfield making their results mixed in determining the turbulence downstream from the combustor depending on which measurement tool they were using. They found that using a fast response sub-miniature pressure transducer was suitable for measuring the axial turbulence spectra of combustor exhaust gasses, but wavenumber spectra were more effective measuring the turbulence characteristics of flows with different velocities. Goebel et al. [7] ran an experiment of combustor turbulence levels under fired and high pressure conditions. Using a Laser Doppler Velocimeter (VDL) they found that the flow conditions had little effect on the turbulence levels and the turbulence levels were mainly due to the swirl within the combustion system. Also, that the length of decay had a large effect on the turbulence levels that the turbine vanes experienced. However, they stated that the results that they generated are difficult to compare to others in this area due to the fact that they conducted their tests within a pressure vessel. One other test that was done in this area was done by Zhang and Gleazer[8]. By using a heat flux probe to measure the turbulence they found that the turbulence exiting the combustor for first stage vanes ranged from $8 \%-12 \%$, depending on the combustor, and a turbulence level of $12 \%-18 \%$ downstream from the first and second stage turbine blades. They determined that the rise in turbulence from the first stage of vanes to the second stage of vanes was due to unsteady wakes generated from the trailing edges of the vanes. Even though most of the data on the measurements of turbulence can by widespread, all of the findings come down to how critical it is to understand turbulence if one wants to accurately predict the heat transfer on a gas turbine vane, especially in the stagnation region of the vane. 


\section{Stagnation Region Augmentation}

The influence of turbulence on the stagnation region heat transfer of turbine vanes has been since at least 1950. Zapp [9] found a correlation between an increase in free stream turbulence and an increase in the heat transfer in the stagnation region of a bluff body. A turbine blade or vane can be an example of a bluff body. Since these early days of experiments there have been many different tests that have found issues with these findings. Recently, Chowdhury and Ames [10] and Kingery and Ames [11] found that for larger leading edges a higher level of turbulence does not always have this effect. This could be due to the effects of increased convection time and the influence of the fluid stain rate on turbulence intensification. Recently, Gandavarapu and Ames [12] reviewed many different pieces of literature on the effect of turbulence on stagnation region heat transfer, and they found many examples that involved turbulence intensity and the correlation between stagnation region heat transfer. Van Fossen et al. [13] did a study on the effect of free stream turbulence, length scale, Reynolds number, and leading edge velocity gradient on stagnation region heat transfer. They measured the heat transfer at the stagnation region of four models at five different turbulence levels. After their tests they came to the conclusion that stagnation region heat transfer augmentation increased with decreasing the length scale, but failed to find the optimum scale. Also, that the stagnation region heat transfer augmentation which was caused by the turbulence was unaffected by a changing velocity gradient. In a similar study Dullenkopf and Mayle [14] found a linear relationship between the laminar stagnation heat transfer and the effective turbulence level. The effective turbulence level that they mentioned is the overall level of the free-stream turbulence accounting for its length scale. By defining the turbulence term in this fashion they found very similar results to Ames and Moffat's [15] study. Sanitjai and Goldstein [16] did a study on the 
effects of free stream turbulence on local mass transfer on a circular cylinder. They performed their tests at multiple Reynolds numbers and turbulence levels and came to the conclusion that the mass transfer changes significantly with turbulence levels. One area of significance that they found was at the stagnation region. They came to the conclusion that with an increasing turbulence level the area near the front of the stagnation region experienced a high level of mass transfer, but at the rear portion of the stagnation region the mass transfer levels return to previous levels. Nix et al. [17] did a similar study to those mentioned above and found that the mean heat transfer levels at a high turbulence, approximately $10-12 \%$, had an increase in heat transfer levels over a baseline test at a low turbulence level by $8 \%$ on the suction surface, and $17 \%$ on the pressure surface of the tested vane. Nix and Diller [18] also did a study on the heat transfer augmentation by free stream turbulence at a stagnation point of a cylinder. They tested at five different turbulence levels and used a hot wire to measure the cross-flow velocity at a range of positions in front of the stagnation point. They found that there was a connection between the surface heat flux and the velocity unsteadiness. This disappeared when the velocity sensor was too far or too close to the surface being measured, which demonstrated that there is a small area that greatly affects the surface heat transfer. They also found that this area is generally correlated with the length scale of the freestream turbulence. Gifford et al. [19] performed a study in a water tunnel facility to examine the physical traits of heat transfer augmentation when affected by freestream turbulence. They found that heat transfer augmentation occurs when the freestream fluid gets swept into the laminar momentum and thermal boundary layer of the heated stagnation surface. An increase in turbulence increases these affects, meaning it has a large effect on the stagnation region heat transfer. 


\section{Endwall Heat Transfer}

Not only do the vanes in gas turbines experience extremely high temperatures, so do the endwalls. Having knowledge of the heat transfer that occurs on the surface of the turbine endwalls and how that effects the vanes would be very beneficial to accurately creating a cooling system for the vane and endwall. Because of this many different experiments have been done to create a better understanding the effects that heated endwalls have on the overall heat transfer in gas turbines. Boyle and Russell [20] performed a study looking at the endwall heat transfer in a linear cascade. They used a liquid crystal measurement technique to help measure the heat transfer. They came to the conclusion that the endwall heat transfer distribution was stongly influenced by the flow through the cascade. At low Reynolds numbers they found the flow to act more like inviscid flow streamlines where at high Reynolds numbers they found the streamlines of the flow to correlate closer to the free stream velocity. Also, that the Stanton number on the endwall changed with the thickness of the boundary layer, meaning a thicker boundary layer led to a higher Stanton number. However, there was no correlation between the boundary layer thickness and the Stanton number contours. Harvey and Jones [21] did a test in an annular nozzle guide vane. They analyzed Nusselt number contours on the endwall and compared them to 3-D predictions. Their results were varied; they came within 10-15\% downstream of the separation line, but upstream of the separation line their predictions were under predicting by a wide margin. Also, they couldn't find a way to predict the high heat transfer at the leading edge. Spencer et al. [22] performed heat transfer measurements on the endwalls of a large annular cascade. They used a transient liquid crystal technique to measure the heat transfer over the endwall of the cascade. They found that the endwall heat transfer increased from the front to the back of their endwall, and the heat transfer differed from the casing and hub endwalls. Kumar et 
al. [23] performed tests on endwall heat transfer for a linear vane cascade. They found that a constant Mach number the Reynolds number effects are felt over the entire endwall. Also, that the effects of turbulence are localized to the endwall near the leading edge of the vane. In another study Radmonski and Thole [24] performed endwall heat transfer tests based on Stanton number at turbulences levels of $0.6 \%$ and $19.5 \%$. They found that the turbulence level has a significant impact on the heat transfer levels an endwall experiences, a higher turbulence level will significantly increase the heat transfer. Recently, Ames et al. [25] did a study in a linear cascade on the effects of turbulence on endwall heat transfer distributions. They ran tests at turbulence levels ranging from $0.7 \%$ up to $14 \%$ at multiple Reynolds numbers. They came to the conclusion that the low turbulence endwall heat transfer contours show strong evidence of secondary flows especially at the higher turbulence levels, which help raise the heat transfer in these areas. As the turbulence levels decrease the evidence of secondary flows affecting the endwall heat transfer seem to dissipate due to the added mixing in the flow. Ames et al. [26] again did a similar study to this but this time they investigated the effects of catalytic and dry low NOx combustor turbulence on endwall heat transfer distributions. In this study their testing parameters were the same as before, and found that their highest levels of heat transfer were found near the leading edge and downstream from the wake, and in the passage at the trailing edge plane. Also, that their results from this test were very similar to the previous tests that Ames [25] had done.

\section{Vane Heat Transfer}

Experiments done on vane surface heat transfer have been very useful in the development of many different prediction methods into the effects of turbulence on laminar region augmentation and surface heat transfer. Hylton et al. [27] and Arts et al. [28] generated vane surface heat transfer measurements at many different turbulence levels. The technique that they 
used and the results that they found have been widely used to help in predictive comparisons over the years. Azad et al. [29] investigated the heat transfer of the area where the stresses are highest, the blade tip regions. They found that these areas of higher stress are a result in a higher heat transfer, along with an increase in turbulence increases the heat transfer in these blade tip regions. Radmonski and Thole [30] studied how turbulent flow convects through vane passages. They found that throughout the vane passage the turbulence level does not decay, but remains at a high level throughout. This phenomenon can lead to high levels of heat transfer in the stagnation regions and regions of laminar flow on turbine blades. Ames [31] studied vane heat transfer data at very high turbulence levels with well documented characteristics, and came to a conclusion that the length scale, Lu, had a significant effect on stagnation region and pressure surface heat transfer. This study also suggested that there was a dependence for pressure surface laminar augmentation similar to that of Dullenkopf and Mayle[14]. Ames et al. [32, 33] investigated a turbine vane under a broad range of Reynolds numbers and turbulence conditions and reconfirmed the conclusions of previous studies. The continued growth of the gas turbine industry had pushed the development of the industry into one where efficiency and reliable power is at the forefront. This means that for industry to evolve so does the understanding of the effects of turbulence on surface heat transfer and the accuracy of the predictive models that are being produced.

The focus of the current study being done is to profile the heat load on an aft loaded vane with a large leading edge. The vane was designed this way to help reduce the overall heat loads that the leading edge would experience and to create a vane with ample amount of space in the leading edge for internal cooling. The aft loaded suction surface was designed to delay transition until further downstream, providing a better environment for film cooling. The current vane heat 
transfer distributions are expected to provide a relevant test case for the improvement of the predictive methods for heat transfer augmentation and transition. 


\section{CHAPTER III}

\section{EXPERIMENTAL APPROACH}

This chapter gives a detailed description of the University of North Dakota's large scale,

low velocity cascade wind tunnel, as well as the construction and experimental procedure used to acquire free-stream pressure and temperature measurements on two different large leading edge instrumented cascade vanes. The wind tunnel is configured in a steady state blown down arrangement and shown in Figure 1. The wind tunnel components, which will be discussed in this section, include the inlet filter box, a blower, two multi-vane diffusers, a heat exchanger, a mixer, a flow straightener, a screen box, a nozzle, the cascade test section, an exit diffuser, and an oblique diffuser. The nozzle component of the wind tunnel is interchangeable. It is used to create different levels of turbulence ranging from $0.7 \%$, using a low turbulence nozzle, to $17.4 \%$, using the new high turbulence generator. All together seven different turbulence levels were tested at three different Reynolds numbers ranging from 500,000 to 2,000,000. 


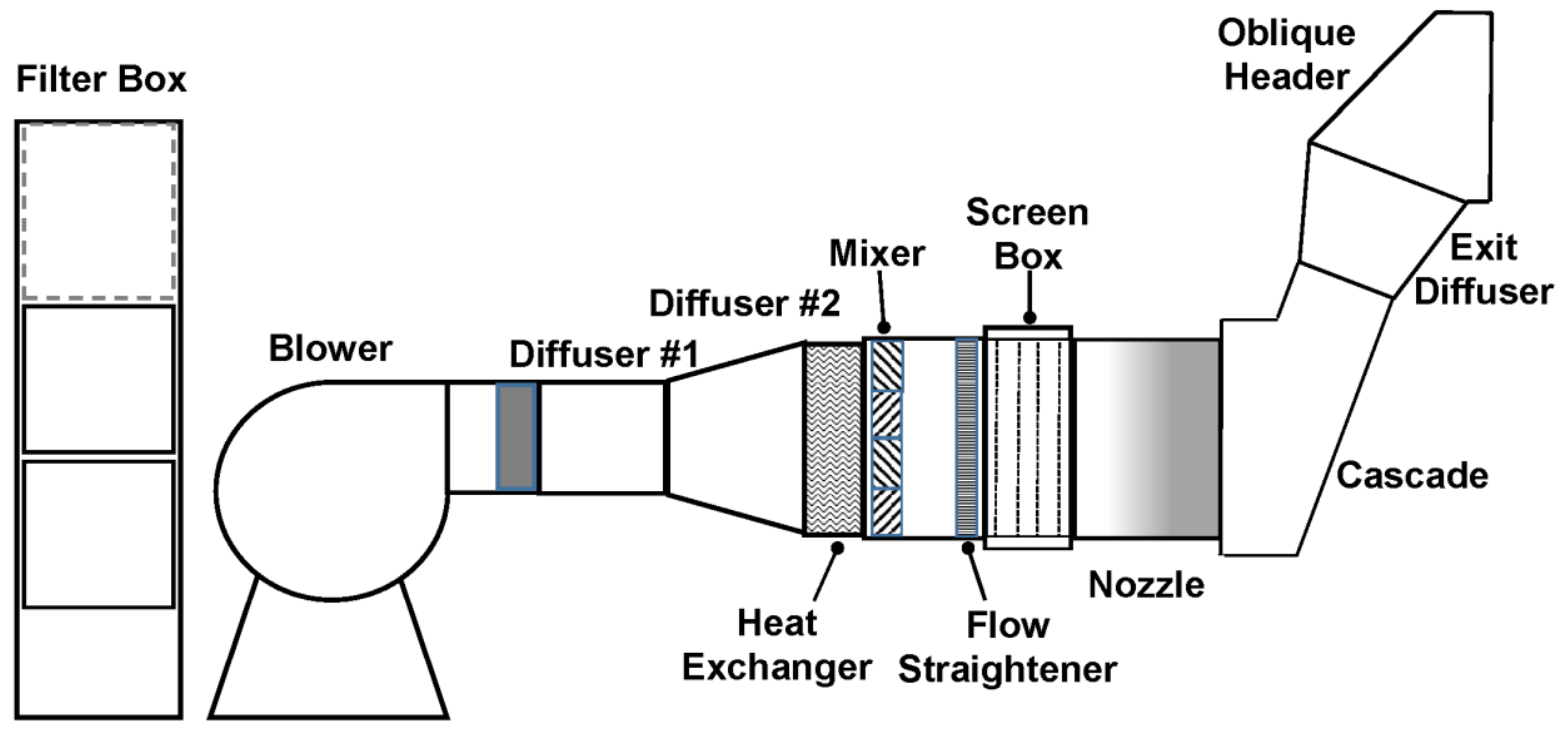

Figure 1. Large scale low velocity wind tunnel

Filter Box

The University of North Dakota's large scale, low velocity cascade wind tunnel is used to force air through the test section in which is being studied. To begin this process the air is first entrained through a filter box system which contains eight different Air Handler 6B640 industrial air filter to catch any particulates that could have been entrained with the air. Each of the filters is 24 inches by 24 inches and has an efficiency of $95 \%$. Filtering all of the particulates from the air is important because if any particulates get into the testing area, they could adhere to the test surfaces, foul hot wires, or clog pressure probes. If the particulates adhered to the testing surface the surface could become rough and alter the characteristics of the flow. If the hot wires or pressure probes became harmed from the particulates the data that is collected could be altered.

\section{Blower}

Once the air travels through the filter boxes it enters the blower. The blower that is used for our experiments is a New York 274-AF blower which can deliver $6.6 \mathrm{~m}^{3} / \mathrm{s}$ of air at a 5000Pa static pressure rise. The blower is powered by a $45 \mathrm{KW}$ electric induction motor which is 
controlled by a Hitachi variable frequency drive. Having the blower attached to the variable frequency drive gives the operator the ability to finely tune the rpms of the blower and lets them keep the Reynolds number close to the desired number. The blower is shown below in figure 2 .

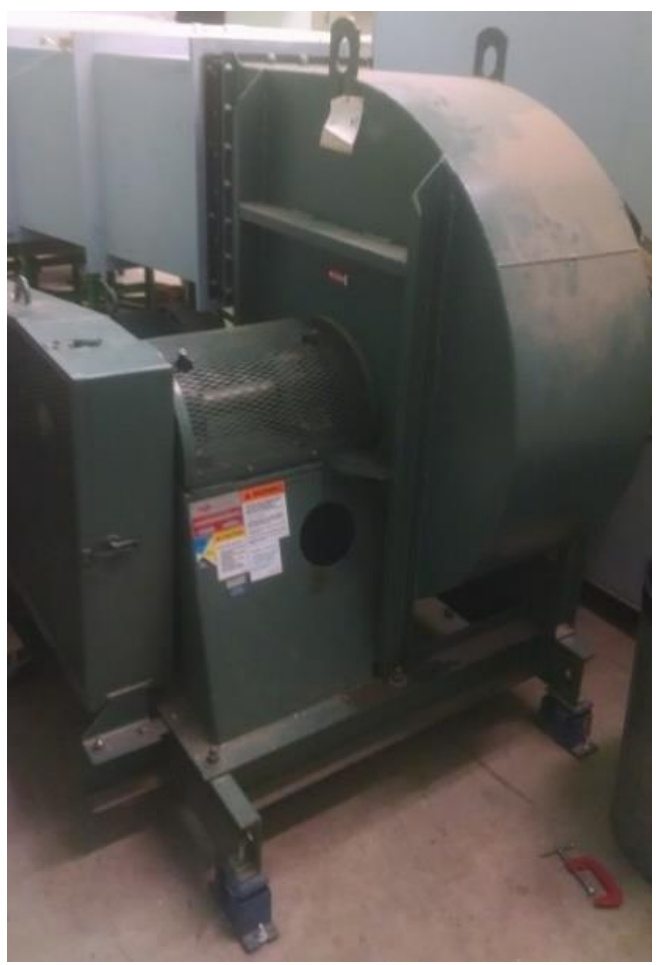

Figure 2. New York Blower used for Wind Tunnel

\section{Diffusers}

After leaving the blower, the air is passed through a two stage multi-vane diffuser that is used to reduce the velocity of the air by expanding the flow and to regain some of the static pressure. The two dimensional diffusers are used to increase the area of the wind tunnel from 16.5 inches by 24.25 inches $\left(400.125 \mathrm{in}^{2}\right)$ to 36 inches by 50 inches $\left(1800 \mathrm{in}^{2}\right)$. This area expansion is accomplished using two stages. The first diffuser expands the width from 16.5 inches to 36.0 inches, while the second diffuser increases the height from 24.25 inches to 50.0 inches. This area expansion helps create a more useable flow for the wind tunnel flow conditioning apparatus. 


\section{Heat Exchanger}

The air that exits the diffuser passes through a heat exchanger that uses a cooling water recirculation system. The heat exchanger is there to remove any of the energy added to the air by the blower. The water for the heat exchanger is stored in a 100 gallon plastic tank and is circulated through the system by a $1 / 2 \mathrm{hp}$ jetted tub pump. At the bottom of the tank there is a $1 \frac{1 / 2}{2}$ inch polyvinyl chloride (PVC) pipe which carries the water from the tank to the heat exchanger. A ball valve is placed after the pump to control the amount of water that enters the heat exchanger. The water exiting the heat exchanger travels back into the large plastic tank. Finally, there is a $1 \frac{1 / 4}{4}$ inch PVC pipe that takes the overflow water from the tank and places it into a drain on the floor. The schematic of the heat exchanger can be seen in figure 3 . The ball valve feature on the heat exchanger is very important because, it allows the operator to control how much energy is taken out of the air depending on how hard the blower is working. At the higher Reynolds numbers more energy needs to be taken out of the air because more was put into it to propel the air at its higher velocity. So, to keep the testing parameters as similar as they can be from run to run more water needs to be cycled through the heat exchanger to cool the air. The system that is in place makes it possible to set a steady inlet temperature within a range of \pm 0.2 degrees Celsius, which helps us create accurate and reliable testing results. 


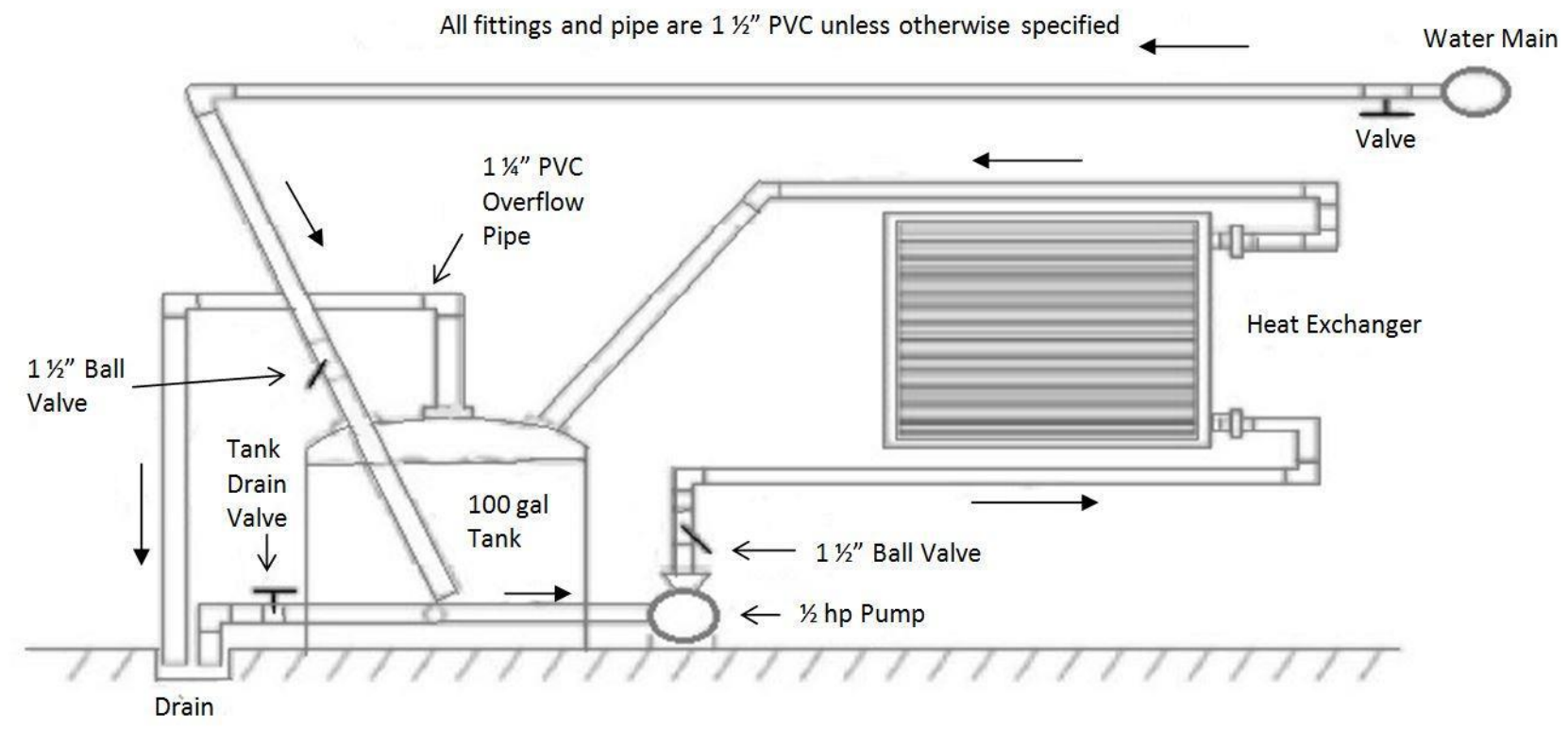

Figure 3. Schematic of wind tunnel heat exchanger [42]

In previous studies it was found that this heat exchanger can experience spanwise temperature stratification when running at higher Reynolds numbers due to two major reasons. First, when running at the higher Reynolds numbers, there is a large amount of cold water being added to the system. Second, the heat exchanger is relatively large, meaning that the water added to the system has time to warm before it travels back to the reservoir. In the high free-stream turbulence conditions this is negated by the high levels of mixing that take place downstream from the heat exchanger. However, in the cases of the lower turbulence levels this can cause some problems, so a flow mixer was added to the system.

\section{Flow Mixer}

The flow mixer that was added to the system is located 13 inches downstream from the heat exchanger. This is the location where the expansion from the heat exchanger compared to the full tunnel height ends. The flow mixer is made from 16 gauge steel and measures 36 inches by 50 inches. It contains ten rows of five fins turned 30 degrees off the plane perpendicular from the flow, causing the flow to be altered 60 degrees either right or left as it travels through the 
mixer, depending on which row the flow goes through. This helps mix out the spanwise temperature differences in the flow. Figure 4 show the flow mixer inside of the wind tunnel.

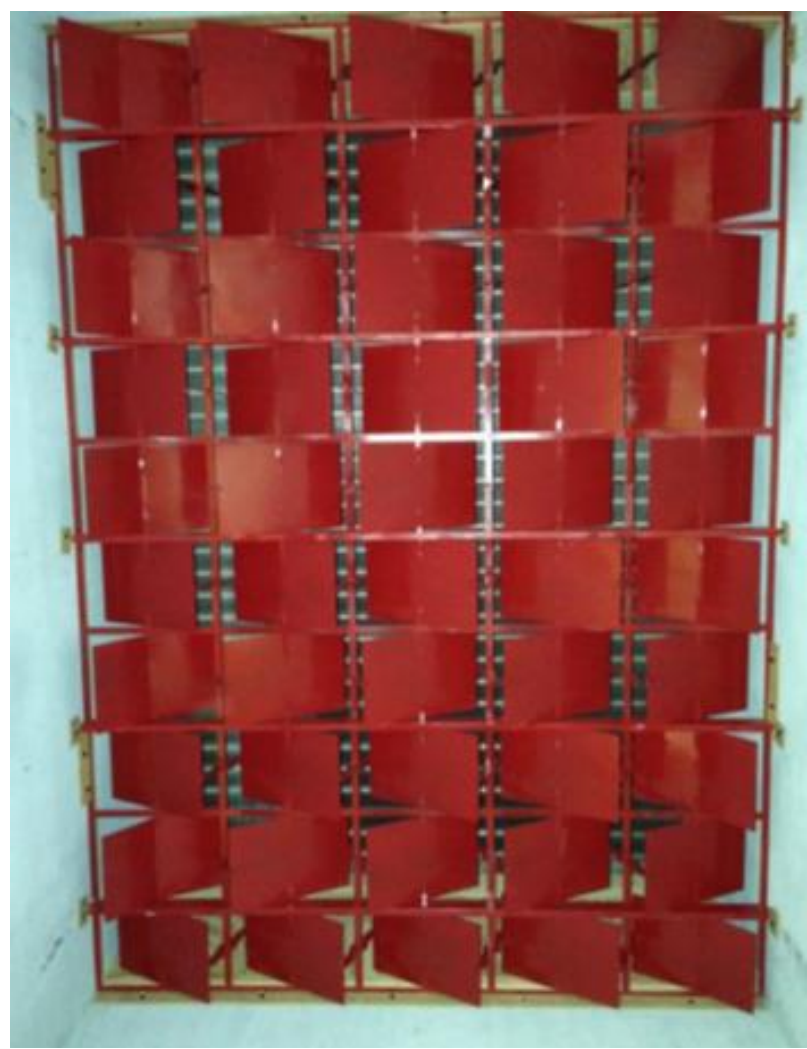

Figure 4. Flow mixer [42]

\section{Straightener}

Since the flow mixer creates significant mixing within the wind tunnel the flow needs to become more uniform before it reaches the test section. To help with this a flow straightener was added to the wind tunnel. The purpose of the straightener is to help negate the mixing that is occurring in the wind tunnel, along with helping increase the uniformity of the flow before it reaches the test section. The flow straightener is made from $1 / 8$ inch diameter honeycomb aluminum with a streamwise length of 2 inches. It can be seen below in figure 5 . 


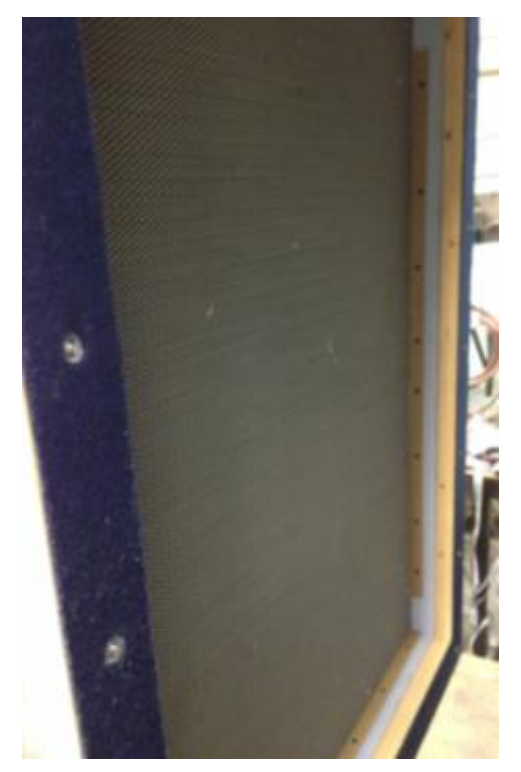

Figure 5. Flow straightener [42]

\section{Screen Box}

Downstream of the straightener the air enters a screen box. This screen box consists of four large nylon screens placed one after the other, each of them spaced 2 inches from the other. These screens produce a uniform resistance to the flow, reducing any velocity variations along with any temperature variations. The screen box is shown below in figure 6 .

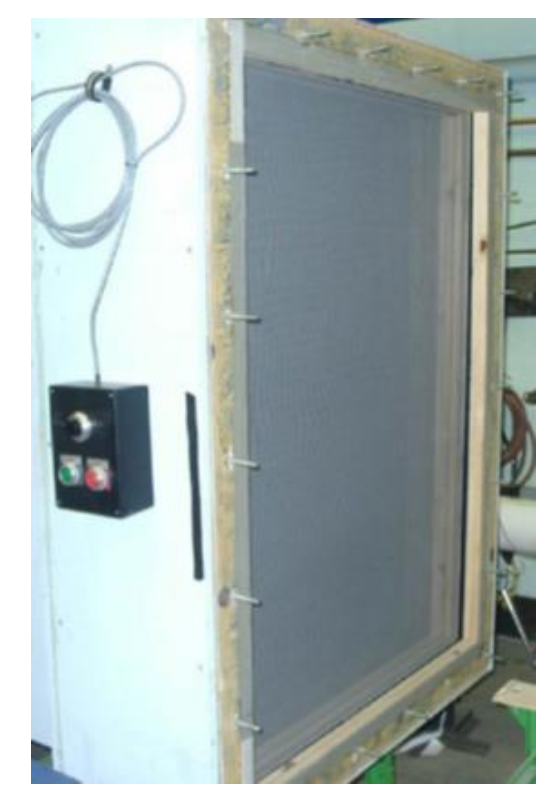

Figure 6. Screen box [42] 


\section{Turbulence Generators}

For this experiment three different nozzles or turbulence generators were used to create seven different turbulence levels. The different turbulence generators were designed to allow a range of turbulence levels from $0.7 \%$ to $17.4 \%$. The three different nozzles that were used during the experiments are a low turbulence nozzle, a mock aeroderivative combustor, and a high turbulence nozzle.

\section{Low Turbulence Nozzle}

The low turbulence nozzle was used to create the four lowest turbulence levels that were used in the testing of the cascade. The nozzle was designed to create a smooth transition through a 3.6 to 1 area reduction. When the cascade was placed directly to the exit of the low turbulence nozzle the turbulence intensity of the flow was $0.7 \%$. The low turbulence nozzle can be seen in figure 7 .

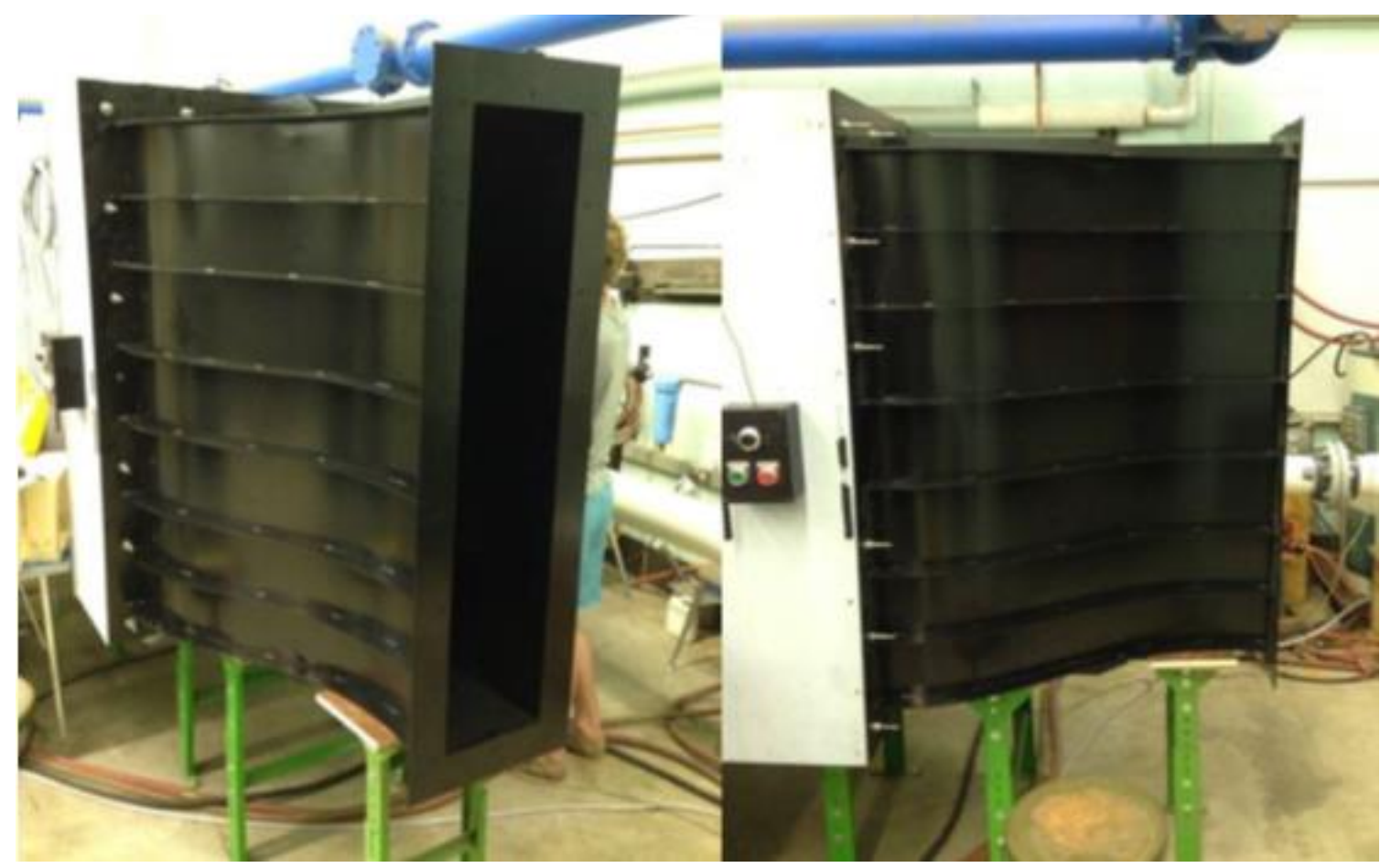

Figure 7. Low turbulence nozzle [42] 
The next three turbulence levels were created using a spool with different grids in placed in the spool to create the different turbulence levels. The spool was placed at the exit of the nozzle and has three different positions where either a large grid or small grid could be placed. The combination of placement and grid design created the different turbulence conditions. The spool is made of $3 / 4$ inch acrylic and maintains a constant 10 inch by 50 inch cross sectional area, identical to the exit area of all three nozzles. A small grid which is made up of 0.25 inch square aluminum bar spaced at 1.25 inches, or five bar widths resulting in a $64 \%$ open area grid can be placed in one of two different positions. A far position, 32 mesh lengths (40 inches) from the test surface, which creates a turbulence level of 3.5\%, or a near position, 10 mesh lengths (12.5 inches) from the test surface, which creates a turbulence level of $7.8 \%$. The small grid near and far configurations can be seen in figure 8 .

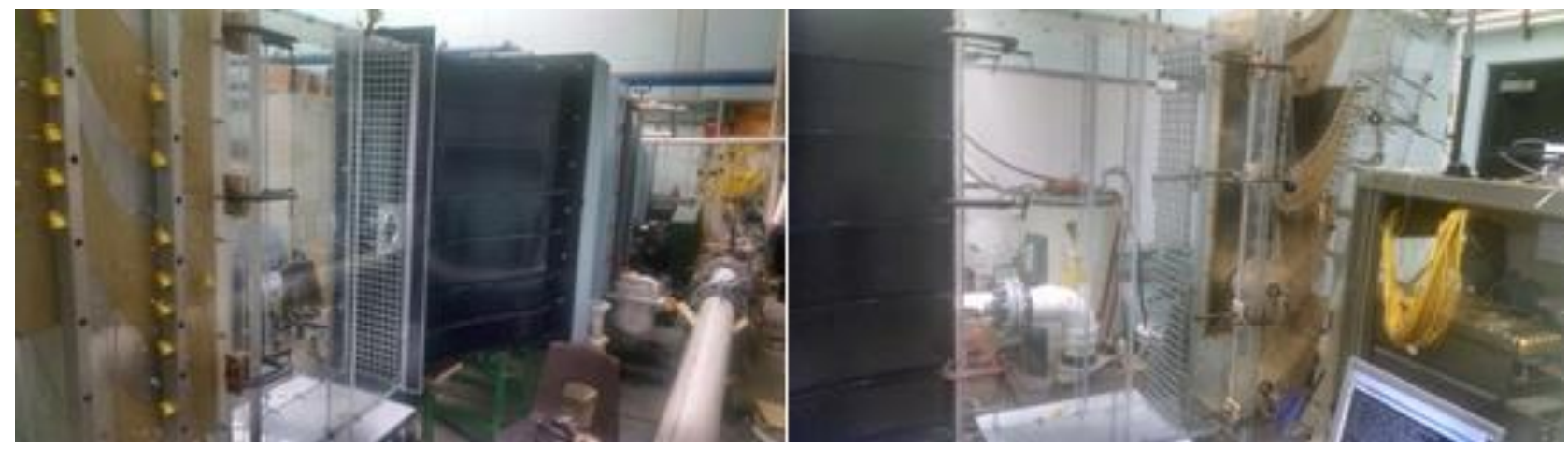

Figure 8. Small grid far configuration (left) and Small grid near configuration (right)

The second grid, the large grid, is made from 0.5 inch square aluminum bars with a five bar width spacing. The grid can only be placed at one location in the spool and it is at a distance of 10 mesh lengths ( 25 inches) from the test surface. This orientation of the large grid and low turbulence generator creates a free stream turbulence of $8.1 \%$. The large grid turbulence configuration can be seen in figure 9. 


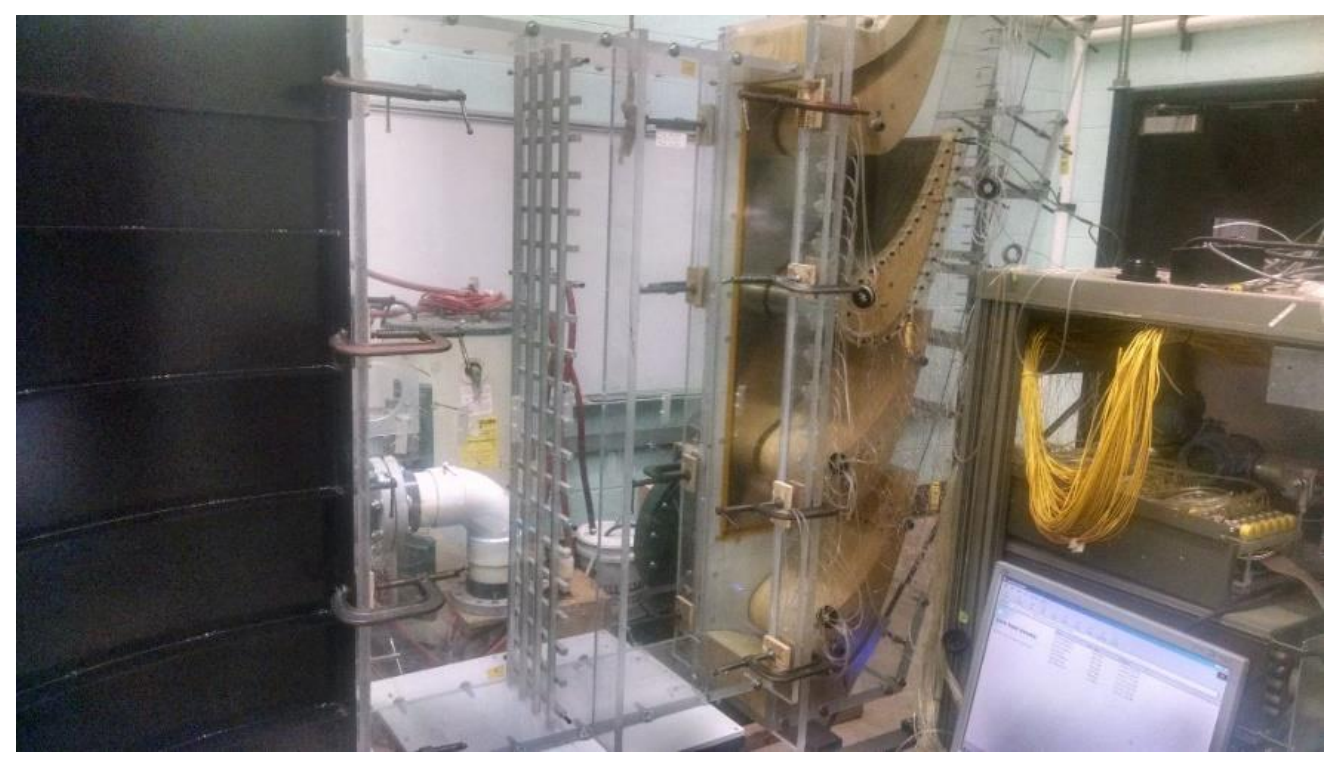

Figure 9. Large grid configuration

\section{Mock Aeroderivative Combustor}

The next two turbulence conditions are created by using a second nozzle. This nozzle, the mock aeroderivative combustor, is a nozzle capable of generating turbulence without either of the grids. This nozzle also had a 3.6:1 area reduction from inlet to end. There are two different configurations that were used during testing. One configuration has the test cascade placed directly against the nozzle, and the other has a decay spool placed between the nozzle and test cascade. The exit turbulence intensity reaches $13.7 \%$ when only the mock aero combustor in place, and when the decay spool in placed after the aero-combustor the turbulence intensity drops to $9.2 \%$. The mock aero combustor can be seen in figure 10 . 


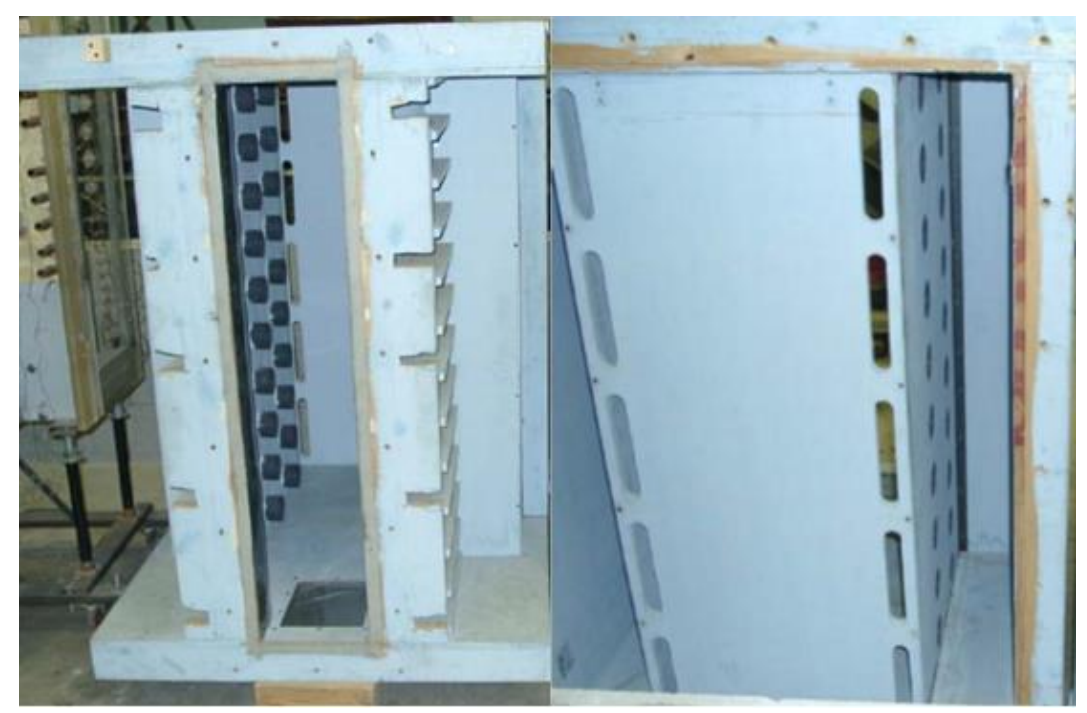

Figure 10. Exit of mock aero combustor (left) and inlet of mock aero combustor (right) [42]

High Turbulence Nozzle

The highest turbulence level is created from our new high turbulence generator, shown in figure 11. It has the same area reduction, as well as exit area as the two other nozzles used in the testing of the cascade. The test cascade is placed in direct contact with this nozzle, and no turbulence creating grids or spools are used with this nozzle. When the high turbulence nozzle is placed in direct contact with the cascade it creates a free stream turbulence of $17.4 \%$. All of the turbulence and flow conditions can be seen in Table 1.

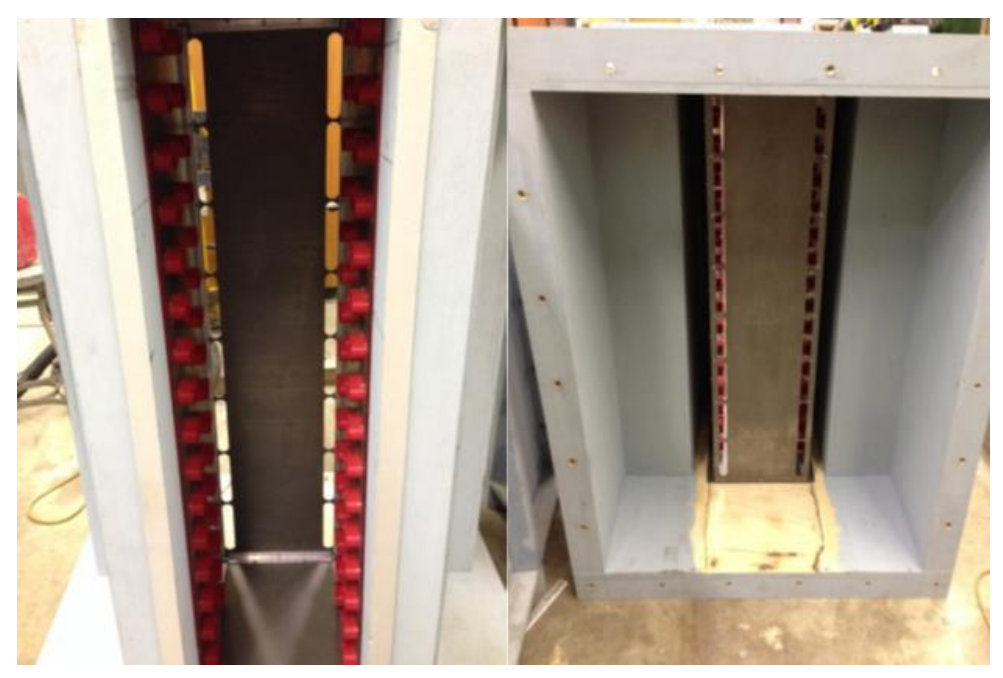

Figure 11. High turbulence generator [42] 
Table 1. Turbulence level characteristics

\begin{tabular}{|c|c|c|c|c|c|}
\hline & $\begin{array}{c}\mathrm{U} \\
(\mathrm{m} / \mathrm{s})\end{array}$ & $\mathrm{Tu}$ & $\begin{array}{l}\mathrm{Lx} \\
(\mathrm{cm})\end{array}$ & $\begin{array}{c}\mathrm{Lu} \\
(\mathrm{cm})\end{array}$ & $\begin{array}{c}\varepsilon \\
(\mathrm{m} 2 / \mathrm{s} 3) \\
\end{array}$ \\
\hline \multirow{3}{*}{$\mathrm{AH}$} & 4.89 & 0.1744 & 3.76 & 6.66 & 13.95 \\
\hline & 9.72 & 0.1744 & 3.91 & 7.22 & 101.3 \\
\hline & 19.34 & 0.1718 & 4.00 & 7.69 & 715.9 \\
\hline \multirow{3}{*}{$\mathrm{AC}$} & 484 & 01235 & 368 & 724 & 441 \\
\hline & 9.11 & 0.1302 & 3.52 & 6.36 & 39.4 \\
\hline & 18.11 & 0.1259 & 3.58 & 7.35 & 241.8 \\
\hline & & & & & \\
\hline \multirow{3}{*}{ ACS } & 4.84 & 0.0883 & 5.08 & 9.03 & 1.30 \\
\hline & 9.17 & 0.0916 & 4.61 & 8.81 & 10.08 \\
\hline & 17.60 & 0.0897 & 4.44 & 9.49 & 62.2 \\
\hline & & 0.0792 & & & \\
\hline \multirow{3}{*}{ GR } & 4.93 & 0.0792 & 2.00 & 3.25 & 2.75 \\
\hline & 9.94 & 0.0798 & 2.04 & 3.34 & 22.41 \\
\hline & 18.95 & 0.0811 & 2.35 & 3.36 & 162.15 \\
\hline & & & & & \\
\hline \multirow{3}{*}{ SG1 } & 4.48 & 0.0808 & 1.51 & 1.78 & 4.00 \\
\hline & 9.12 & 0.0781 & 1.61 & 1.85 & 29.4 \\
\hline & 17.87 & 0.0792 & 1.12 & 1.97 & 216.0 \\
\hline \multirow{3}{*}{ SG2 } & 4.70 & 0.0384 & 2.38 & 3.81 & 0.232 \\
\hline & 9.08 & 0.0350 & 1.73 & 3.23 & 1.49 \\
\hline & 17.61 & 0.0348 & 2.13 & 2.85 & 12.1 \\
\hline \multirow{3}{*}{ LT } & 4.96 & 0.0069 & 8.12 & 127.0 & 0.00005 \\
\hline & 9.65 & 0.0076 & 5.02 & 154.5 & 0.00038 \\
\hline & 18.71 & 0.0060 & 3.58 & 15.50 & 0.0139 \\
\hline
\end{tabular}




\section{Cascade Test Section}

After passing through any of the three nozzles and the many different turbulence generating configurations the air enters the large scale linear cascade. The cascade is based on a modern mid-sized industrial gas turbine and created on an eleven to one scale. The goal is for the cascade to depict the airfoils and endwalls in an industrial gas turbine. The current cascade being tested is designed with a four vane, three passage configuration. The cascade contains many features that are in place to ensure two-dimensional aerodynamic data that are accurate and repeatable. There are four vanes cast from an epoxy, three slave vanes which are bolted into the cascade, as well as an interchangeable vane. The cascade also includes a heated endwall, three endwall coolers, a row of inlet pressure taps and a row of exit pressure taps, two adjustable tailboards, and an upper and lower bleed flow adjustment. The entire configuration of the cascade can be seen in figure 12 .

The four vane, three passage linear cascade was fabricated from 1 inch thick acrylic. This allowed the operator to see into the cascade when testing was being completed along with provide a rigid structure that doesn't conduct heat. Detailed dimensions of the cascade are shown in Appendix A. One of the important features of the cascade are the 30 inlet and exit pressure ports. The inlet pressure ports are placed at one-quarter axial chord upstream from the leading edge of the vanes. Similarly, the exit pressure ports are placed at one-quarter axial chord downstream from the trailing edge. Along with the pressure ports that are on the endwall, there are 10 probe access ports that were drilled through the endwall. These ports were designed to help monitor the inlet pressure and temperature conditions of the cascade. The cascade turns the flow between 74 and 75 degrees before the flow exits the cascade and enters the exit diffuser. 


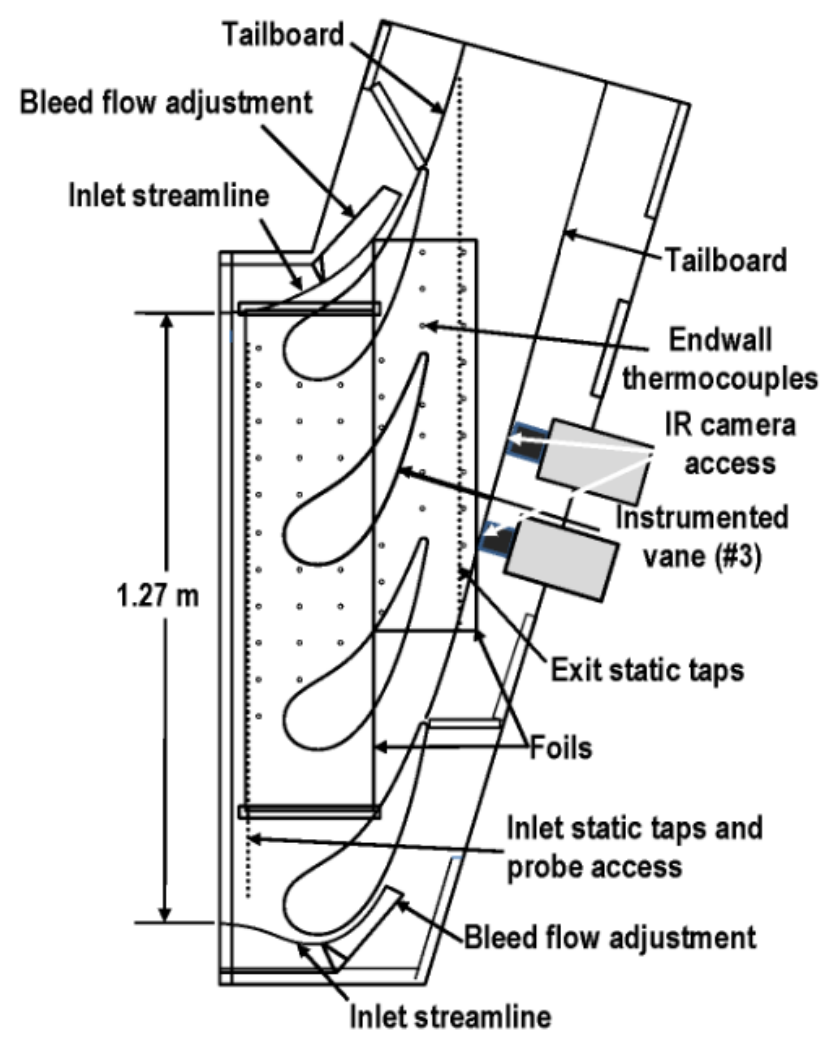

Figure 12. Layout of cascade

Bleed Flow Adjustments

The cascade that was constructed has two bleed flow adjustments, an upper bleed flow and a lower bleed flow. These bleed flow fixtures were designed to mimic the boundary conditions that the vanes themselves provide in the cascade. They were constructed with an isocyanurate foam core and covered with a very thin, 1/64 of an inch, and flexible epoxy fiberglass (G-10) board. Before the foam core was covered 10 individual pieces were routered to size using a mold created earlier in the process. They were then glued together and cut down to size to ensure that the vane would be 10 inches after the flexible epoxy fiberglass board was attached to it. The shapes of the vanes were designed to keep the inlet flow that entered the cascade along the projected streamlines and to attempt to keep the flow as uniform as possible in the cascade. The bleed flow vanes are adjustable to control the flow rates along the top and the 
bottom vane along with help in maintaining the desired streamlines on the upper and lower slave vane.

A slot was cut into the bottom bleed flow adjustment and nylon rods were attached to the back of the fixture to help adjust the flow. The top bleed flow adjustment has a piece of adjustable acrylic placed on top of the vane which is there to perform the same function as the cut out slot on the lower vane. This acrylic piece has the ability to slide in and out to meter the flow around the vane and help create the desired streamlines. Both the upper and lower bleed flow vanes can be seen below in figure 13 .

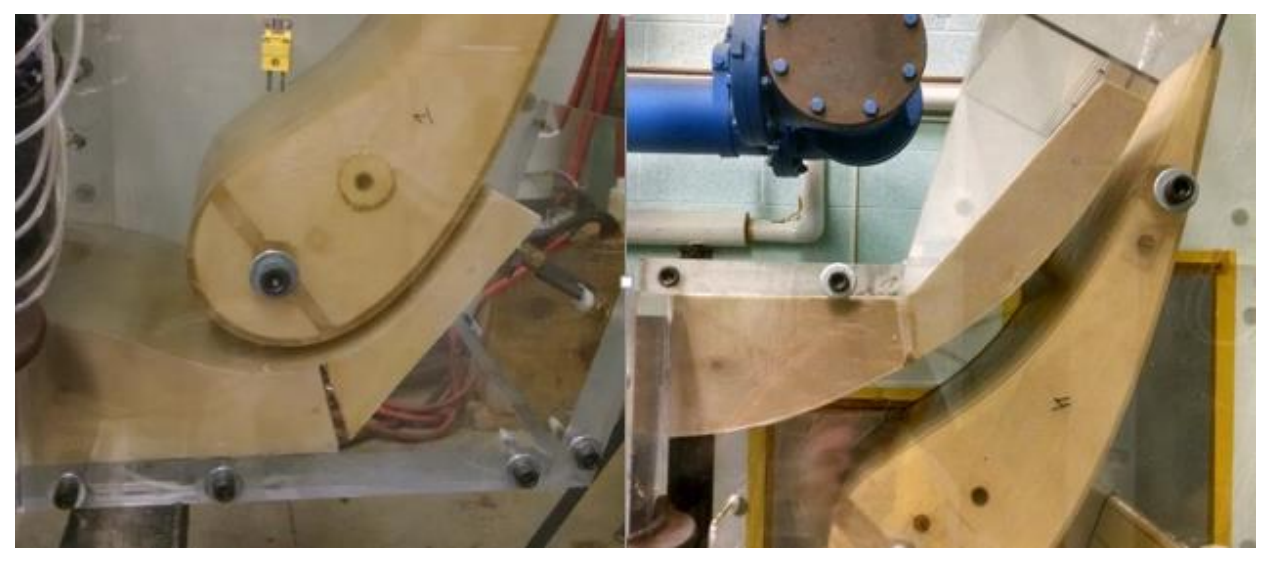

Figure 13. Lower tailboard (left) and upper tailboard (right)

\section{Exit Tailboards}

The two exit tailboards were attached to the trailing edges of the top and bottom slave vanes in the cascade. Their purpose is to ensure that there is exit periodicity in the flow. The tailboards were made to be flexible to ensure that they would be placed on the streamlines of the flow, accounting for the growth of the boundary layer along the tailboards themselves.

The tailboards were fabricated from 3/16 inch polycarbonate. They were attached to the top and bottom slave vane through $1 / 4$ inch wide by $1 / 2$ inch deep groove that was machined into each of the vanes. The tailboards were inserted into the grooves and fastened in place with a 
RTV silicone sealant. The tailboards were made adjustable by attaching $3 / 8$ inch nylon rods, which were threaded, to the backs of the tailboards using $3 / 4$ inch acrylic spacers that were glued in place and machined to have two threaded holes placed six inches from each other. The bottom tailboard had five different areas where the nylon rods could be placed and help aid in the placement and adjustment of the tailboard. The top tailboard had two sections where the nylon rods could attach and help place the tailboard. Attached to each of the two tailboards was a sheet of polycarbonate which sat at a 90 degree angle to the edge of the tailboard. These were glued there to help aid in the adjustment of the tailboards at the exit of the cascade.

The bottom tailboard had a feature designed into it which allows an IR camera to be placed there and view the suction side of the heat transfer vane. Two windows were drilled into the tailboard, which can be seen in figure 14. They provide an area where an IR camera lens, made from zinc selenide, can be placed allowing pictures of the heat transfer on the suction surface of the vane to be taken. This allows the flow patterns of the suction surface to be seen visually, instead of relying on the calculations to see the flow pattern.

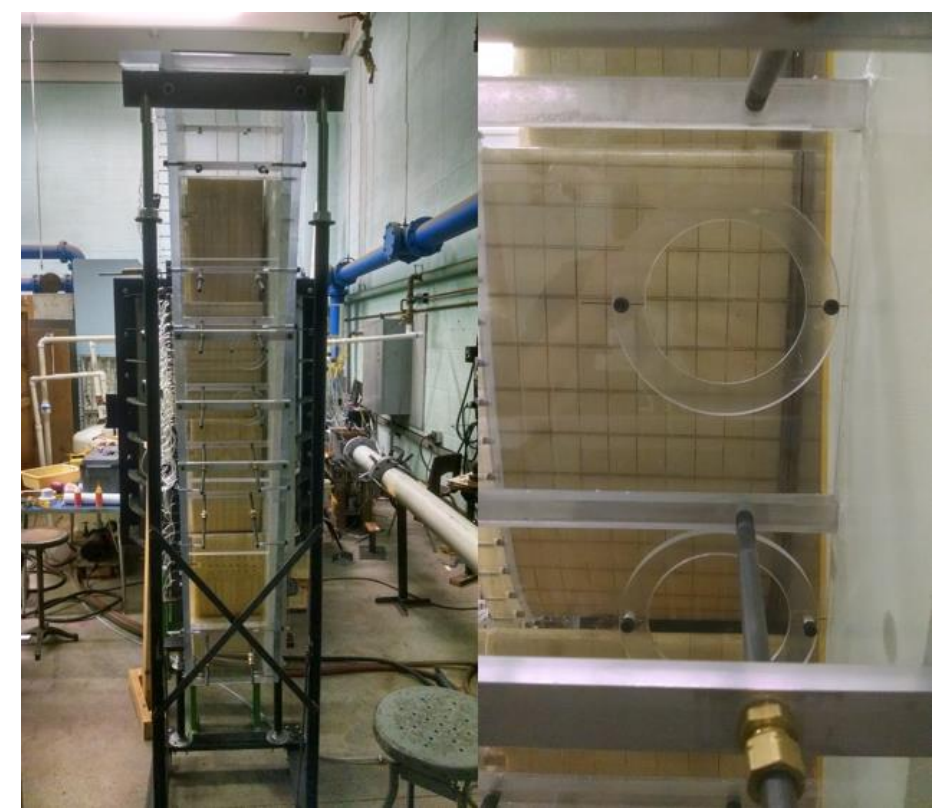

Figure 14. The tailboard with adjustable nylon rods (left) and tailboard windows (right) 


\section{IR Camera Mounting System}

In order to take the IR camera pictures the camera itself has to be mounted onto the cascade. The IR camera mount has two different locations where it can be placed, one for the upper window on the tailboard and one for the lower window. It was constructed so the angle of the camera can be adjusted in all three directions, the $\mathrm{x}, \mathrm{y}$, and z-direction. This way it can be assured that the camera is located perpendicular to the vane, and is at the desired length from the vane for the picture to be in focus. The IR camera mounting system can be seen in figure 15 .

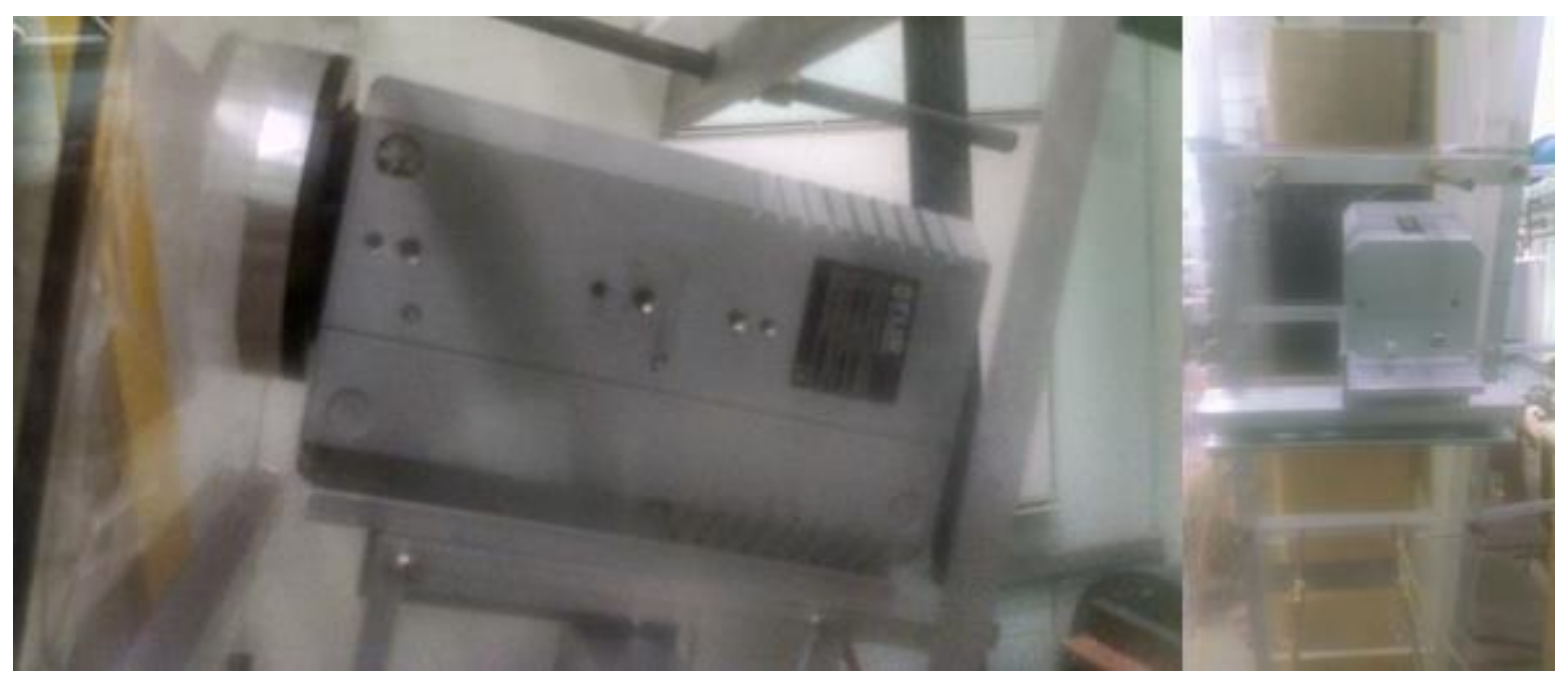

Figure 15. IR camera mount side view (left) and back view (right)

\section{Turbine Vanes}

The vane that was used in the linear cascade was designed to have a relatively large leading edge and an aft loaded suction surface. The vanes leading edge was designed to have a 5.2 inch diameter on a line that passes through the stagnation point on the vane. Another reason the large leading edge design was chosen was to help reduce the heat transfer levels in the leading edge area of the vane, along with creating more room for the leading edge to be internally cooled. The aft loaded suction surface was designed to accelerate the boundary layer, therefor minimizing the growth of the boundary layer, and delaying transition of the flow. This 
aft loaded design is also there to help film cooling on the suction surface. By keeping the flow laminar as long as possible there will be less mixing in the flow, helping the coolant stay along the vane instead of being mixed away.

The vanes that are being tested in the cascade have a chord length of 19.590 inches and are vertically spaced 15.152 inches apart from each other. The leading edge to trailing edge distance for each vane when it is placed in the cascade is 11.198 inches. This means that each vane is placed at an angle of 54.42 degrees. After each vane was cast, they were cut down to a precise height of 10.0 inches and placed in the cascade. Three of the four vanes were bolted into the cascade; these vanes are known as slave vanes. The other vane, which is the third vane from the bottom, can be easily inserted and removed from the cascade through a window cut into the endwall. This vane is known as the instrumented vane. There are two different instrumented vanes for the cascade, one of them is a pressure vane the other a heat transfer vane. More information about the fabrication of the vanes can be found in Appendix B.

\section{Pressure Vane}

The pressure vane is one of two instrumented vanes that were constructed for testing in the cascade; it can be seen in figure 16 . The vane has 40 pressure taps placed on the outer edge of the vane at locations determined to obtain an optimal vane surface pressure distribution. The pressure taps were constructed from 1/16 inch brass tubing, and they were placed 1/16th of an inch below the surface of the vane, meaning that they were glued to the foam core of the pressure vane. Brass barb fittings were soldered onto the ends of the pressure taps to help with the ease of assembly for the tubing of the pressure taps. To obtain the pressure distribution along the surface of the vane, 0.040 inch holes were drilled into the brass tubes at the mid span of the vane. This location ensured that the flow, which the pressure taps captured had little to no disturbance from 
the endwalls of the cascade. A concentration of the pressure taps were located near the stagnation region of the vane, due to the fact that the pressure is rapidly changing in this area and more pressure taps are needed to capture the flows behavior in this area. In areas that the pressure drop was more linear the pressure ports could be spaced further from one another.

Table 2. Pressure tap locations

\begin{tabular}{|c|c|c|c|c|c|}
\hline number & surface dist (in) & surface dist (in) & number & surface dist (in) & surface dist (in) \\
\hline 1 & 0.535 & -18.351 & 21 & 20.506 & 1.620 \\
\hline 2 & 1.925 & -16.961 & 22 & 21.171 & 2.285 \\
\hline 3 & 3.259 & -15.627 & 23 & 22.108 & 3.222 \\
\hline 4 & 4.577 & -14.309 & 24 & 23.009 & 4.123 \\
\hline 5 & 5.825 & -13.061 & 25 & 23.870 & 4.984 \\
\hline 6 & 7.259 & -11.627 & 26 & 24.767 & 5.881 \\
\hline 7 & 8.580 & -10.306 & 27 & 25.834 & 6.948 \\
\hline 8 & 10.016 & -8.870 & 28 & 26.916 & 8.030 \\
\hline 9 & 11.109 & -7.777 & 29 & 28.248 & 9.362 \\
\hline 10 & 12.223 & -6.663 & 30 & 29.624 & 10.738 \\
\hline 11 & 13.343 & -5.543 & 31 & 30.917 & 12.031 \\
\hline 12 & 14.407 & -4.479 & 32 & 32.478 & 13.592 \\
\hline 13 & 15.501 & -3.385 & 33 & 34.018 & 15.132 \\
\hline 14 & 16.411 & -2.475 & 34 & 35.576 & 16.690 \\
\hline 15 & 17.313 & -1.573 & 35 & 37.108 & 18.222 \\
\hline 16 & 17.999 & -0.887 & 36 & 38.717 & 19.831 \\
\hline 17 & 18.437 & -0.449 & 37 & 40.250 & 21.364 \\
\hline 18 & 18.886 & 0.000 & 38 & 41.545 & 22.659 \\
\hline 19 & 19.356 & 0.470 & 39 & 42.805 & 23.919 \\
\hline 20 & 19.849 & 0.963 & 40 & 0.000 & -18.886 \\
\hline
\end{tabular}




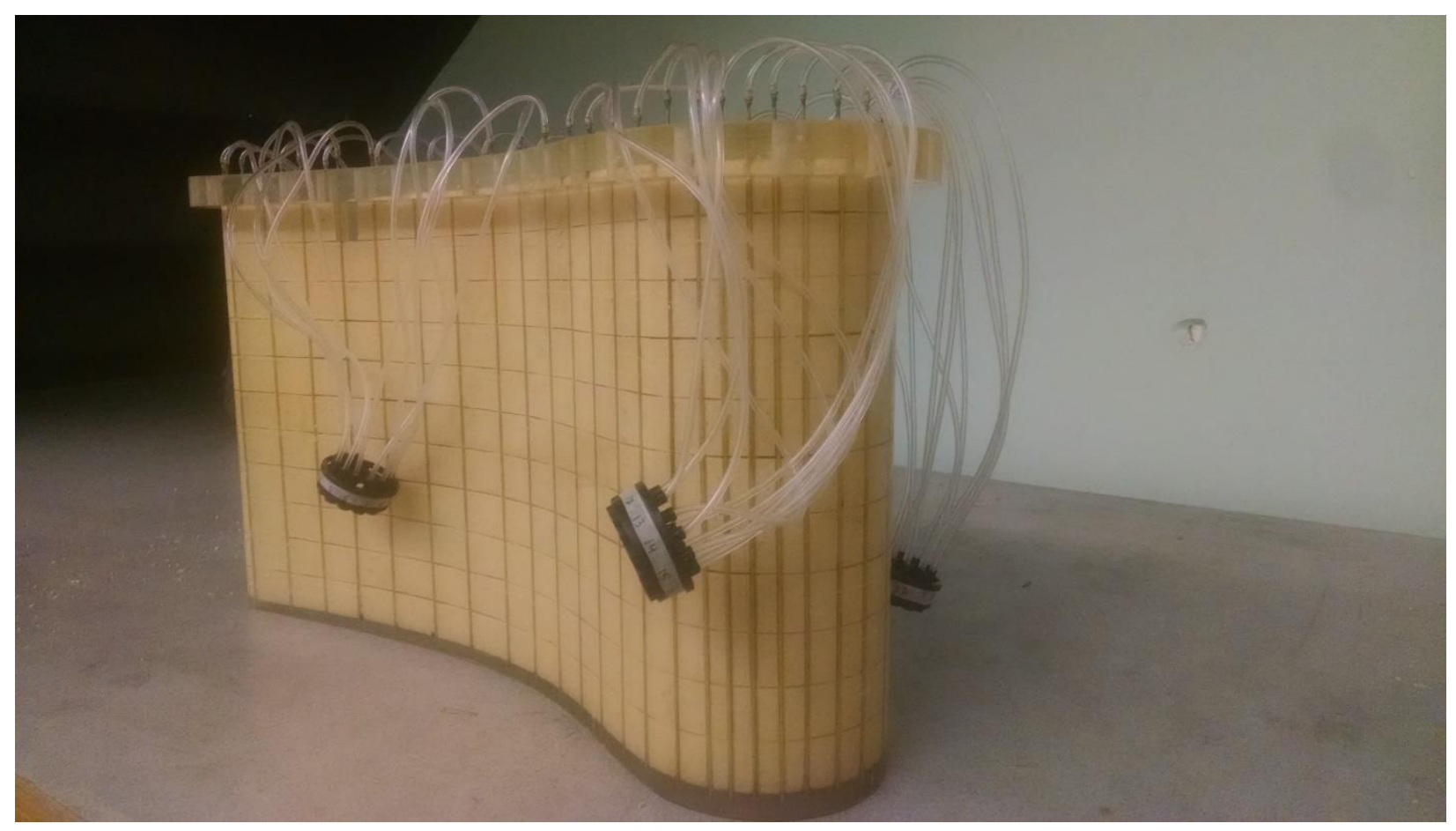

Figure 16. Pressure vane

\section{Heat Transfer Vane}

The second instrumented vane is the heat transfer vane, which can be seen in figure 17. This vane was cast with 55 fine wire type $\mathrm{K}$ thermocouples located at the mid span of the vane. The thermocouples were placed in the vane to obtain a full surface temperature distribution over the vane. The vane was then wrapped with 9.93 inch by 42 inch constant heat flux Inconel foil. The foil was fabricated commercially and is a $0.023 \mathrm{~mm}$ thick (0.00091 inches). The foil also has a $0.05 \mathrm{~mm}$ thick (0.00197 inches) layer of Kapton which allows the foil to be adhered to the surface of the vane. Two copper bus bars are soldered onto the ends of the foil to evenly distribute the current throughout the foil. These bus bars were placed into grooves that were machined onto the pressure and suction sides of the trailing edge of the vane. This was in an attempt to minimize any aerodynamic blockage that could occur from having a bump on the surface of the vane. 
Table 3. Vane thermocouple locations

\begin{tabular}{|c|c|c|c|c|c|c|c|}
\hline number & location (in) & number & location (in) & number & location (in) & number & location (in) \\
\hline 1 & 1.027 & 16 & 13.423 & 31 & 21.494 & 46 & 33.133 \\
\hline 2 & 1.818 & 17 & 14.161 & 32 & 22.147 & 47 & 34.06 \\
\hline 3 & 2.667 & 18 & 14.798 & 33 & 22.706 & 48 & 35.27 \\
\hline 4 & 3.537 & 19 & 15.422 & 34 & 23.397 & 49 & 36.559 \\
\hline 5 & 4.531 & 20 & 16.019 & 35 & 24.047 & 50 & 37.443 \\
\hline 6 & 5.555 & 21 & 16.799 & 36 & 24.677 & 51 & 38.726 \\
\hline 7 & 6.126 & 22 & 17.532 & 37 & 25.429 & 52 & 39.815 \\
\hline 8 & 7.237 & 23 & 17.99 & 38 & 26.167 & 53 & 41.054 \\
\hline 9 & 8.065 & 24 & 18.351 & 39 & 26.892 & 54 & 41.862 \\
\hline 10 & 8.795 & 25 & 18.797 & 40 & 27.487 & 55 & 42.465 \\
\hline 11 & 9.887 & 26 & 19.207 & 41 & 28.449 & & \\
\hline 12 & 10.736 & 27 & 19.631 & 42 & 29.394 & & \\
\hline 13 & 11.641 & 28 & 20.12 & 43 & 30.081 & & \\
\hline 14 & 12.132 & 29 & 20.616 & 44 & 31.17 & & \\
\hline 15 & 12.866 & 30 & 21.023 & 45 & 31.947 & & \\
\hline
\end{tabular}




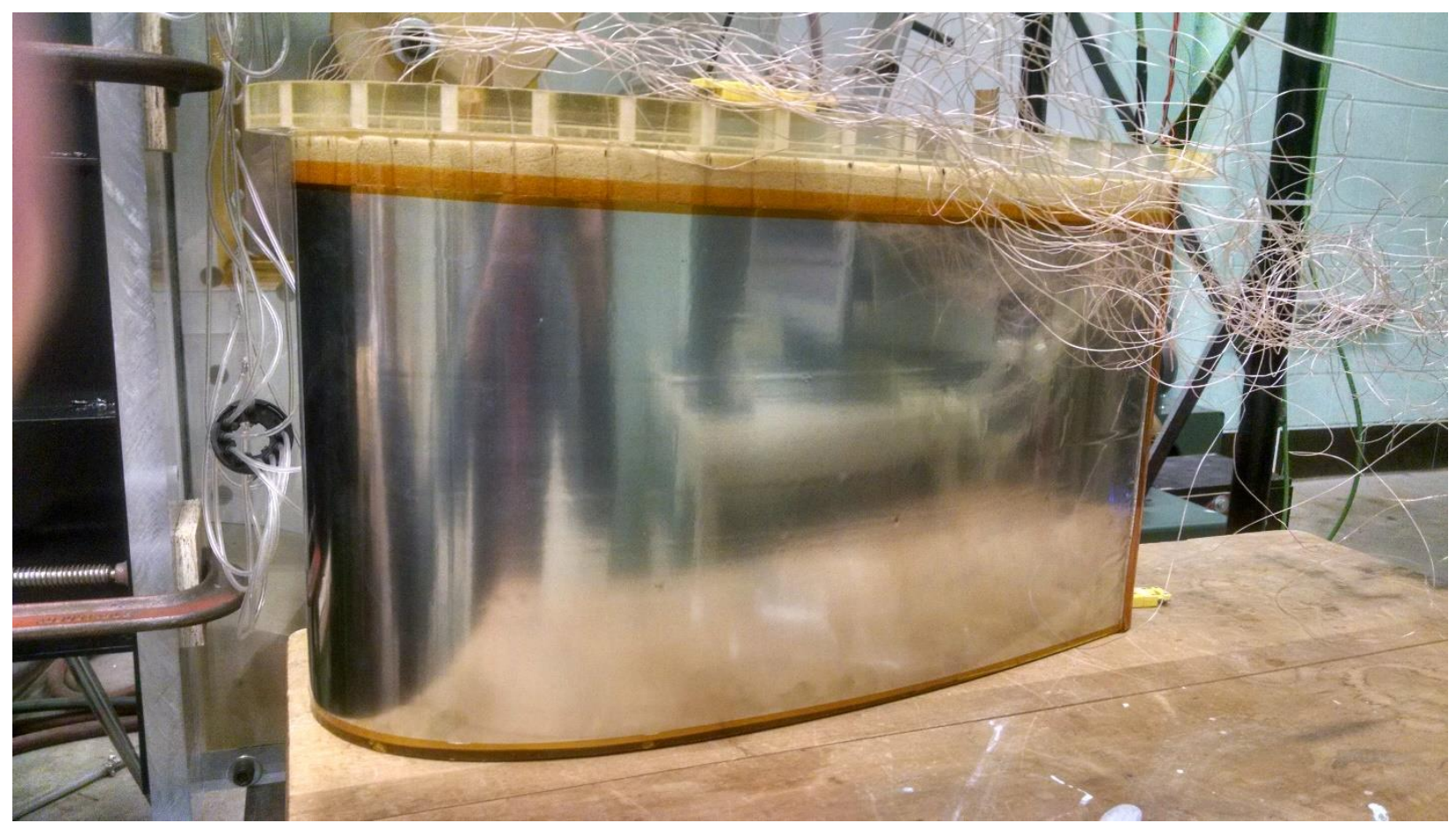

Figure 17. Heat transfer vane wrapped in Inconel foil

To further study the effects of heat transfer on the vane, the heat transfer vane was

painted matte black to accommodate an IR camera. The purpose of the camera was to see the effects of secondary flow patterns on the local vane heat transfer and if there were any significant differences from the endwall flow at the different turbulence levels and Reynolds numbers. The vane was painted matte black using an acrylic paint to achieve the desired emissivity of 0.96.

The painted heat transfer vane can be seen in figure 18. An airbrush was used to paint the surface to achieve a quality finish for the vane to not alter any of the flow patterns around the vane. Gold dots were also painted on the surface of the vane in a 2 inch by 2 inch grid, starting $3 / 4$ of an inch from the edge of the foil on the suction surface of the vane and $1 / 2$ of an inch from the bottom of the vane. The dots were placed on the surface of the vane to help reference where on the vane the heat transfer was located when analyzing the data. 


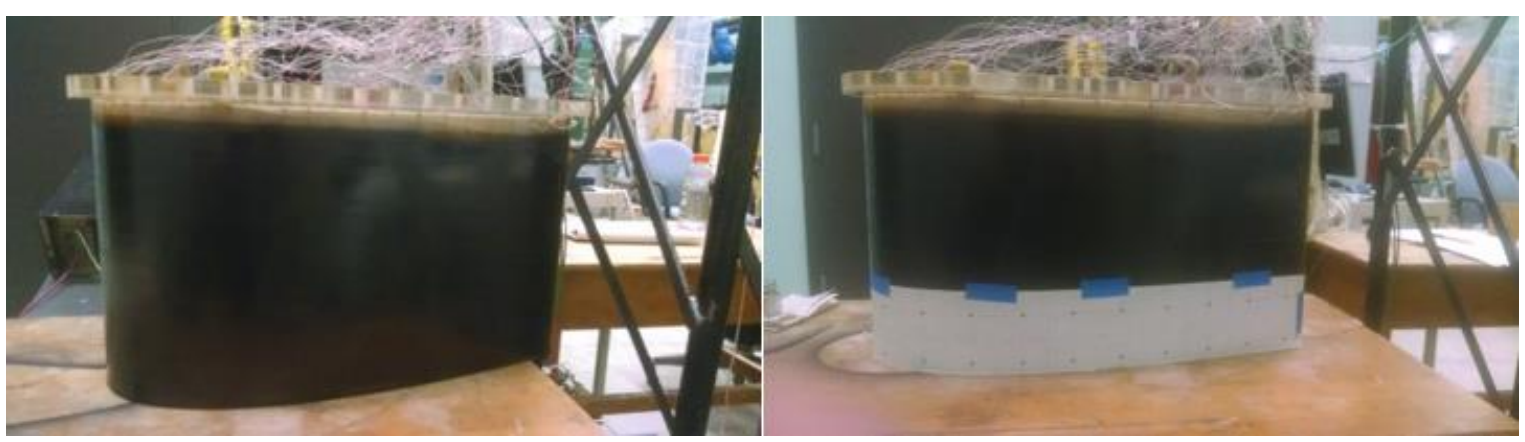

Figure 18. Painted heat transfer vane (left) painted vane with dot location grid (right)

\section{The Endwall}

The cascade was built with two distinct endwalls, a near endwall and a far endwall. The near endwall, which I will describe first, was constructed with the pressure taps and access ports drilled into the surface. The 30 inlet pressure ports, placed at one-quarter chord upstream from the leading edge plane, were in place to ensure good inlet flow uniformity. Similarly, the 30 exit pressure ports, located one-quarter chord downstream from the trailing edge plane of the vane, were placed to help establish the exit periodicity of the flow exiting the cascade. Each of the pressure ports were constructed by drilling a $1 / 8$ inch hole vertically through the acrylic wall until it was approximately 0.05 inches from the end of the acrylic endwall. Then a 0.040 inch drill bit was used to penetrate the surface of the endwall. This created a port that the pressure reading was taken from. The pressure taps themselves were made from telescoping brass tubes that were soldered together. Each individual port has a pressure tap that was made from three different one inch long brass tubes. The three brass tubes that the telescoping pressure ports were made from were first a 1/8 inch diameter tube, followed by a 3/32 inch diameter tube, and finally a 1/16 inch diameter tube. These brass tubes were soldered together and a barbed tube fittings were soldered onto the end, creating an easy way to attach and detach the pressure tubes from the cascade. They were then placed into the holes that were previously drilled into the endwall of the cascade, and epoxied in place. 
The 10 access ports that were drilled into the endwall of the cascade were $3 / 4$ of an inch in diameter and filled with acrylic plugs. These plugs were machined to have a $3 / 4$ inch diameter portion, which is 1 inch in length, and a 1.25 inch diameter portion machined to a length of a half of an inch. This way when the plugs were inserted into the cascade they were stopped when the smaller diameter was flush with the inner surface of the endwall, thus creating little to no disturbance in the flow. The ports were placed into the design of the cascade to allow the inlet total pressure and inlet total temperature of the flow to be monitored. The instrumentation used to collect the inlet total pressure was designed with a 90 degree turn ensuring that it was in line with the streamlines of the inlet flow. The pressure probe also had a 45 degree angle internal bevel cut out of the tip of the probe to help reduce its sensitivity for misalignment. The temperature probe was inserted through a 1/16 inch brass tube to ensure the placement of the fine wire type $\mathrm{K}$ thermocouple in the middle of the cascade, the brass tube also helped increase the rigidity of the thermocouple. This thermocouple was placed in the cascade to record the total inlet temperature of the flow. The near end wall can be seen in figure 19. 


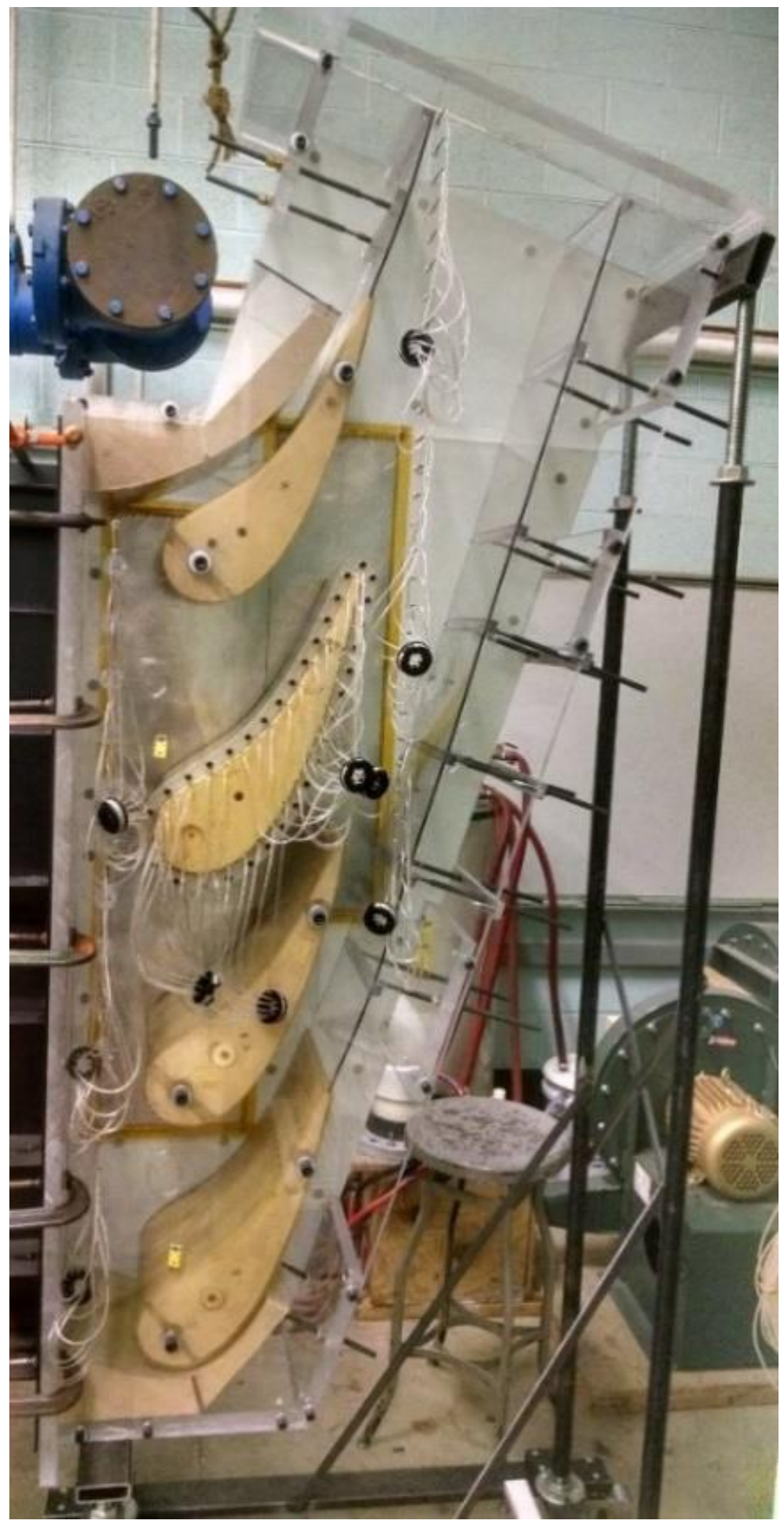

Figure 19. Cascade near endwall 
Along with these features the near endwall included a window cut into the surface, which the two different instrumented vanes to be easily switched in and out of the cascade depending on which one is being tested at that time, the vane window can be seen in figure 20 . The window was cut to a 1/32 inch larger radius than the size of the vanes positioned in the cascade. This way the instrumented vanes can be placed in the same location each time one is switched out for the other, ensuring accuracy between the different tests that were conducted.

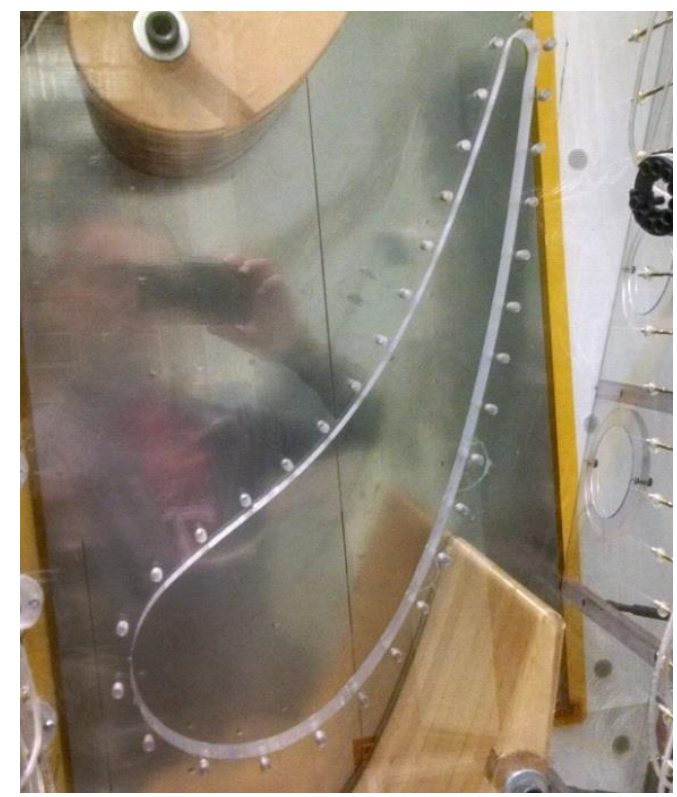

Figure 20. Vane window for instrumented vanes

The far endwall was composed of two different layers, and can be seen in figure 21 . The outer layer is made completely of the 1 inch thick acrylic. Two 1 inch by 2 inch aluminum bars are bolted onto the endwall to help increase its rigidity over the area of the cascade where the constant heat flux foil were placed. This increased rigidity helps the cascade from shifting in any way due to the overall weight of the cascade, and harming the constant heat flux foils in any way. The inner part of the endwall was composed of both the 1 inch thick acrylic and 1 inch thick isocyanurate foam, which provides insulation to the rest of the cascade from the constant heat flux foils. Inside the foam three end wall coolers, or heat sinks, were placed to help keep the 
temperature of the endwall and the temperature of the surface under the vanes constant throughout the testing procedures. More information on the construction of the endwall heat transfer system can be found in Appendix C. The foam and the endwall coolers were then covered with a thin sheet of epoxy fiberglass board which was placed over the foam so the constant heat flux foils could be installed onto the endwall surface. When the constant heat flux foils were being placed on the endwall, the bus bars which are attached to the end of the foils were placed into the two grooves that were machined into the endwall. This attempted to make the entire surface of the endwall level. This procedure was done for both the large foil (42.5 inches by 10 inches) and the small foil (32 inches by 8 inches). After the foils were attached to the endwall a high temperature tape was placed on the edges of the foils to help limit disturbances in the flow. Under the constant heat flux foils 48 thermocouples of known location, were placed on the surface of the endwall to help provide an accurate temperature profile of the two passages surrounding the instrumented vane. 


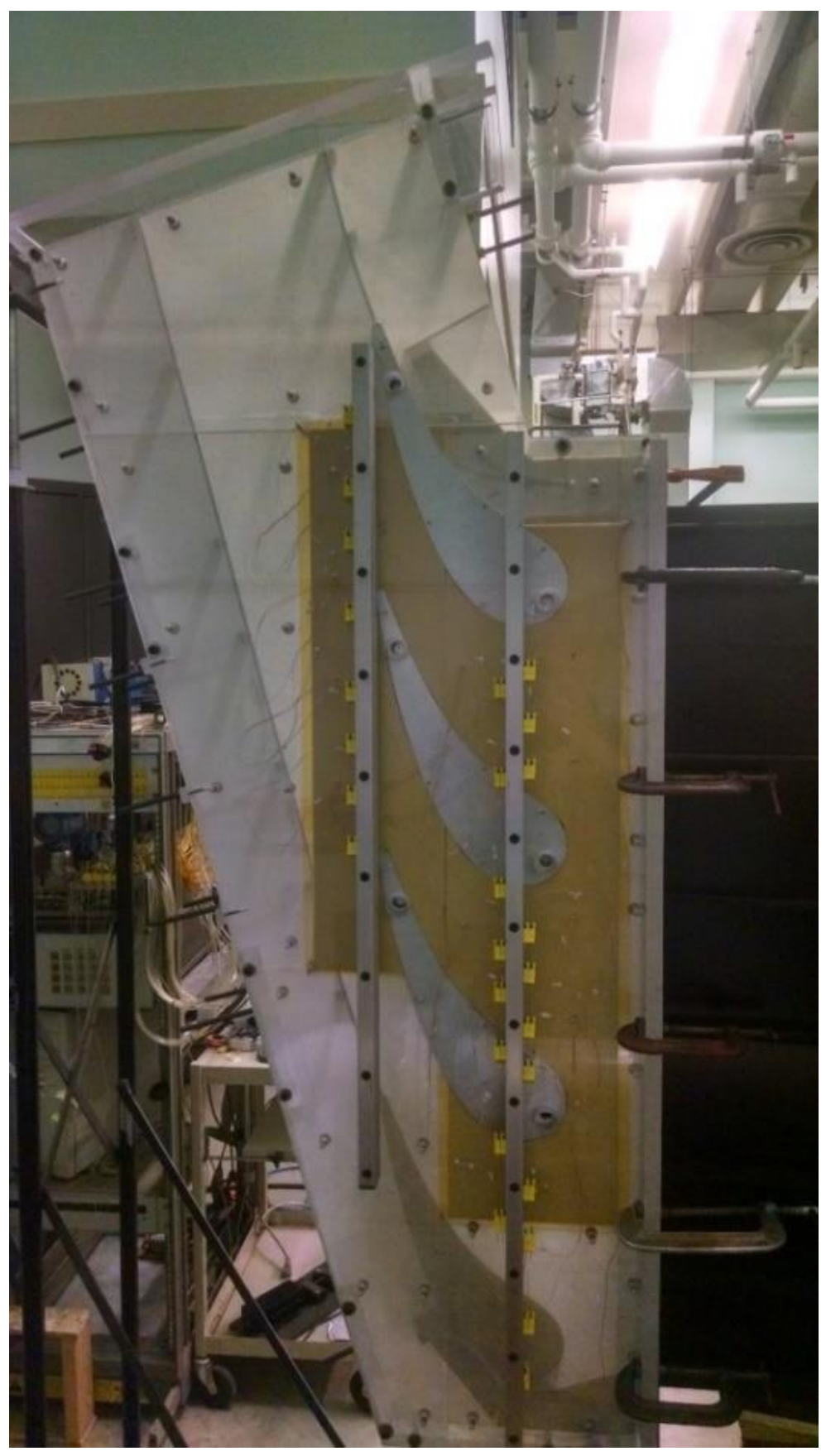

Figure 21. Cascade far endwall 
Table 4. Endwall thermocouple locations

\begin{tabular}{|c|c|c|c|c|c|c|c|c|}
\hline Number & X (in) & Y (in) & Number & X (in) & Y (in) & Number & X (in) & Y (in) \\
\hline 1 & 2.964 & 52.043 & 17 & 6.186 & 31.012 & 33 & 15.743 & 57.286 \\
\hline 2 & 2.92 & 49.38 & 18 & 6.14 & 28.205 & 34 & 15.706 & 54.271 \\
\hline 3 & 2.932 & 46.393 & 19 & 6.161 & 25.058 & 35 & 15.78 & 47.686 \\
\hline 4 & 2.92 & 43.363 & 20 & 9.344 & 49.385 & 36 & 15.775 & 45.17 \\
\hline 5 & 2.923 & 40.383 & 21 & 9.376 & 46.337 & 37 & 15.729 & 42.126 \\
\hline 6 & 2.928 & 37.176 & 22 & 9.376 & 43.324 & 38 & 15.714 & 39.136 \\
\hline 7 & 2.951 & 34.087 & 23 & 9.392 & 34.106 & 39 & 18.93 & 60.281 \\
\hline 8 & 2.962 & 31.083 & 24 & 9.424 & 31.052 & 40 & 18.92 & 57.298 \\
\hline 9 & 2.964 & 28.103 & 25 & 9.445 & 28.05 & 41 & 18.974 & 54.261 \\
\hline 10 & 2.933 & 25.071 & 26 & 12.534 & 51.431 & 42 & 18.942 & 51.251 \\
\hline 11 & 2.946 & 22.035 & 27 & 12.509 & 48.417 & 43 & 18.926 & 48.218 \\
\hline 12 & 6.153 & 49.262 & 28 & 12.548 & 45.877 & 44 & 18.924 & 45.23 \\
\hline 13 & 6.124 & 46.228 & 29 & 12.558 & 36.2 & 45 & 18.976 & 42.264 \\
\hline 14 & 6.132 & 43.199 & 30 & 12.519 & 33.155 & 46 & 18.971 & 39.22 \\
\hline 15 & 6.123 & 40.202 & 31 & 12.533 & 30.647 & 47 & 18.907 & 36.198 \\
\hline & 6.121 & 34.035 & 32 & 15.726 & 60.226 & & & \\
\hline & & & & & & & & \\
\hline 16 & & & & & & & & \\
\hline
\end{tabular}

\section{Exit Diffuser}

After passing through the linear vane cascade the air enters the exit diffuser, which directs the air up toward the ceiling, and into the oblique diffuser which is placed on top of the exit diffusor. The purpose of the exit diffuser is to regain static pressure along with decreasing the velocity of the exiting air. For this diffuser a two-dimensional, three vane, four-passage design was used. The exit diffuser was designed to reduce the velocity of the exiting air by half and regain as much static pressure as possible. The outer shell of the diffusor is made from 1 
inch thick acrylic and was designed to match the size of the exit of the cascade. To adapt to the changing exit area of the cascade the diffusor has movable outer walls that are made from 3/16 inch thick polycarbonate. The three inner vanes were cut from 1/8 inch aluminum sheeting and placed in grooves cut into the sides of the walls in the diffusor. The vanes were taped in place to help ensure there rigidity during the tests. The exit diffusor can be seen in figure 22 .

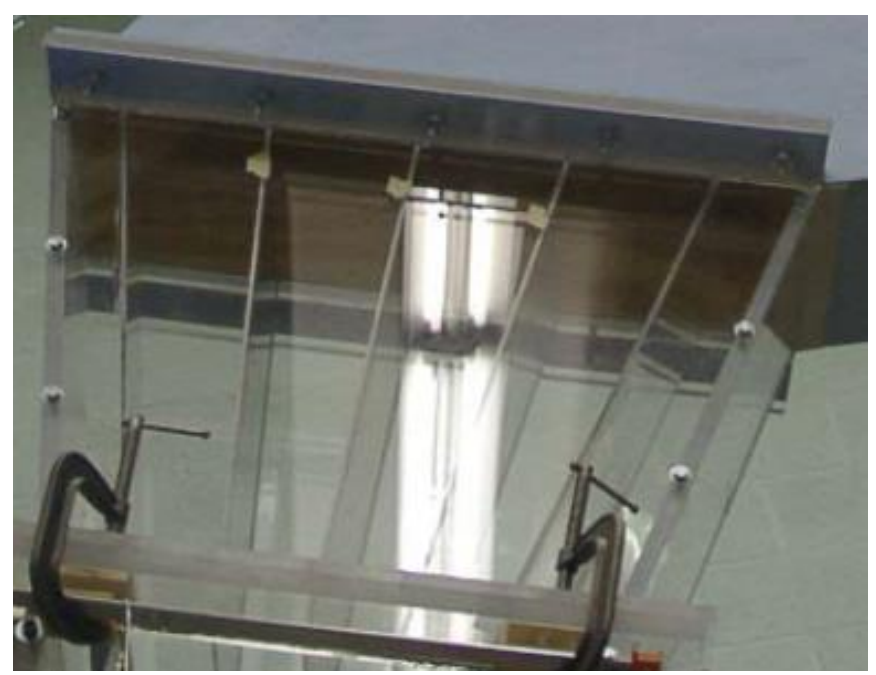

Figure 22. The exit diffusor [43]

Oblique Diffuser

After the air has exited the diffuser the speed at which it is traveling was deemed to be too high for safety purposes. Also, the air coming out of the linear cascade was directed toward the ceiling and more specifically the lighting fixtures hanging down from the ceiling. Taking these light fixtures down was not an option because without them the lighting in our testing area would have caused safety problems and when taking pictures to document different milestones of testing on the cascade. Therefore, an oblique diffuser, shown in figure 23 , was added to the cascade to once again slow the air exiting the cascade, and redirect the air flow 90 degrees ensuring that it wouldn't hit the light fixtures above. The oblique diffuser was designed with eight vanes and nine passages which rotated the air 90 degrees as the air traveled through the 
entirety of the diffusor. It was constructed out of $1 / 2$ inch $A B$ plywood, to ensure that it would be light enough to easily place on top of our cascade. We felt that it was necessary for the oblique diffusor to be light because our cascade is approximately 12 feet tall, and for the ease of assembly a lighter oblique diffusor would be better.

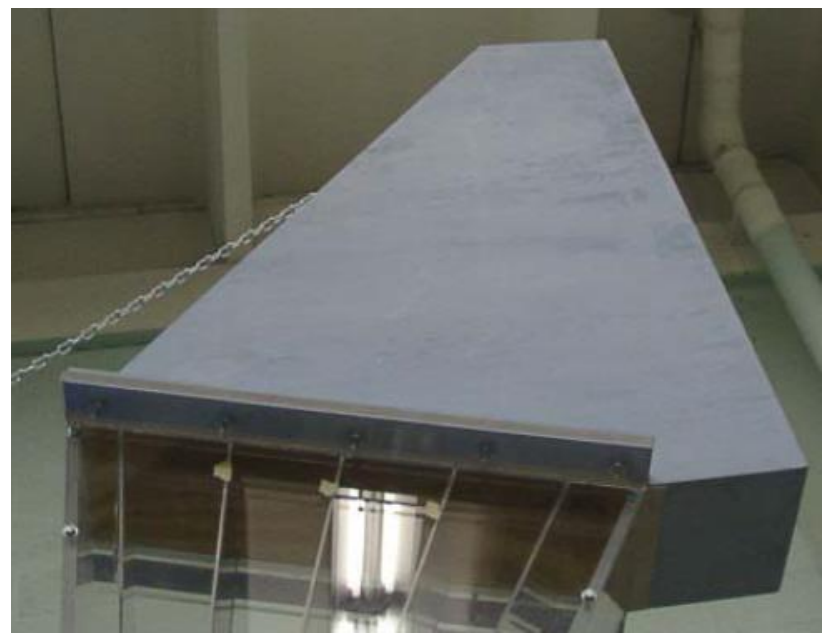

Figure 23. The oblique diffusor [43]

The Data Acquisition System

Data was acquired for this project with a Hewlett Packard 3497A data acquisition system. This system is capable of reading 100 channels of DC voltage measurements. The system was interfaced to a Dell OptiPlex GMT 590 computer to acquire the data that was taken.

\section{Power Supply}

A power supply was attached to the heat transfer vane and also the endwall heat transfer foils for later test to provide the power to the Inconel foils. For the main power supply a 0.001 ohm precision constant shunt resistor was connected to the power supply to help accurately monitor the power supplied to the foils in the cascade. Current was sent to the Inconel foils via 10 AWG stranded wire. The power supply allowed the current and voltages to be adjustable which allowed the temperatures to be raised or lowered depending on which turbulence level and Reynolds number was being tested. When testing the effects of endwall heat on the vane heat 
transfer distribution a second power supply was used. The only difference between this power supply and the main power supply was that a $0.005 \mathrm{ohm}$ precision shunt resistor was connected to this power supply.

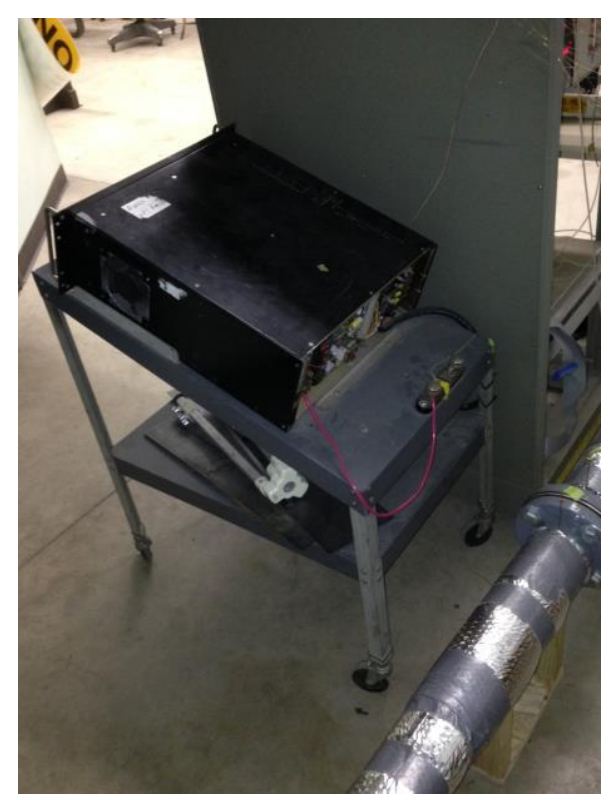

Figure 24. Power supply

\section{Pressure Measurements}

The pressure measurements that were taken for this project were acquired through two different Rosemount pressure transducers. The smaller one measured differential pressures up to $250 \mathrm{~Pa}(0.036 \mathrm{Psi})$, where the larger one measure differential pressures up to $5000 \mathrm{~Pa}(0.725 \mathrm{Psi})$. Both of the transducers have an accuracy of $\pm 0.1 \%$ of the full scale that they are measuring. The transducers were connected to a pressure sensor board which composed of a 48 12VDC pressure solenoids, 1-44 connected to the low side of the transducers and 45-48 connected to the high side. 


\section{Temperature Measurements}

All of the temperature recordings for this project were taken from type $\mathrm{K}$ thermocouples composed of chromel and alumel. Each of the thermocouples used were cut to length, approximately six feet for the ones connected to the heat transfer vane, and approximately four feet for the ones placed on the endwall. The thermocouples placed on the endwall needed wire jumpers to reach the data acquisition tower. The wire that was used was a 36 gauge wire purchased from Omega. The wire consisted of a chromel part and an alumel part which were welded together into a bead using a Therm-X thermocouple welder. This bead was then placed on the surface of the foil where the temperature was to be recorded. The other end of the wire was attached to a male type K thermocouple plug. All of the male plugs were then inserted into the front of our data acquisition tower, which can be seen in figure 25 . To ensure the accuracy of the temperature measurements all thermocouples were referred to an ice bath through a passive constant temperature junction. The ice bath thermocouple was placed in a glass tube, which had mineral oil on the bottom to help give a uniform temperature inside the tube. The tube was then attached to the top of a thermos, so the glass tube could be placed inside the thermos but also have the thermos sealed to maintain its temperature throughout the entire testing procedure. The ice bath had to be changed every two days to ensure the accuracy of the results. 


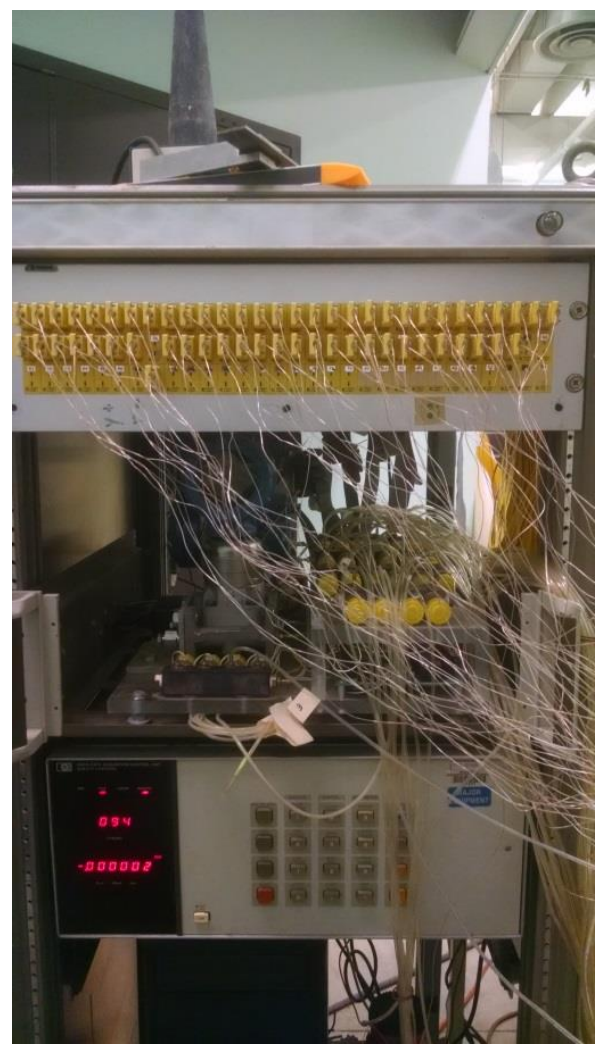

Figure 25. Data acquisition tower

\section{Procedure}

The procedure for acquiring data for the wind tunnel had several steps. First, the blower had to be turned on and the barometric pressure within the room had to be taken. When that was done, the quick basic program FATVNHTP or FATVNSS could be turned on and the barometric pressure could be entered into the program to zero the pressure sensors. When the pressure sensors were zeroed the program opened itself. At this point, depending on what Reynolds number was being run the pump had to be turned on and the fresh water valve had to be opened to activate the heat exchanger and maintain a steady inlet temperature for the system. In the case of a Reynolds number of 500,000 this did not need to be done, because the heat added to the air by the blower was not sufficient enough to alter the inlet temperature. For all other Reynolds numbers this process had to occur. The blower ran until steady state occurred for the system at 
the given Reynolds number being tested. Once steady state was reached then the two different types of tests could occur.

The first tests that were run were the pressure tests. These helped establish that the cascade was set up correctly and gave us the inlet, exit, and vane pressure distributions within the cascade. To run each one of these tests a PCV quick disconnect which was hooked up to the pressure sensors had to be attached to the desired pressure ports that were being tested. The inlet and exit pressure distributions were made up of 30 ports, so only three of the four quick disconnect were used. To take one of the pressure tests the desired number had to be entered into the program. Typing in the number 3 when the program was running had the system to take inlet pressure distributions, the number 4 had the system take exit pressure distributions, and the number 5 had the system take a vane pressure distribution. For the system to take a pressure reading it opened one of the ports, starting at one, for twenty seconds to settle the pressure over that time span. Then it closed that port and moved on to the next one until all of the pressure ports were cycled through. When the program was finished recording all of the pressures it wrote the file to the computer where it could be furthered analyzed.

The second type of data that was taken was the heat transfer data. This data could be taken with the endwall heating system on and off. For each case an adiabatic data set was recorded at steady state for the given test conditions. Then the heaters were tuned on. For the heaters to be turned on the system had to be at steady state with air blowing over the foils so they would not overheat. After the heaters were turned on to their desired voltages and currents, which were monitored by the program, the system again had to reach steady state. The same process was done with the endwall heat was on and off, the only difference with the endwall heat on is that the smaller foil on the endwall surface had its own power supply that had to be set and 
monitored. When the system was at steady state and the vane was at the proper temperature, a minimum temperature difference of approximately 5 degrees Celsius when compared to the inlet total temperature and no more than a 15 degrees Celsius difference anywhere on the vane, the temperature distribution of the vane could be taken. This was option 10 in the program. Once the data were taken it was transferred onto the computer where it could be further analyzed.

A third and final testing procedure was done to record the IR camera data. This process was similar to that of the heat transfer recordings, but the program was modified to monitor the experiment. This program not only monitored the vane surface temperatures, but also the temperatures of the endwall and footprint coolers. The process was still the same as before, the system had to reach steady state before any pictures could be taken. When this occurred the IR pictures could be taken of the suction surface of the vane. When one Reynolds number was completed the procedure occurred for the next one until the process was completed for all Reynolds numbers at that given turbulence level. When the process was completed for no endwall heat, the endwall heater was turned on and the process started all over again. This process used the FATVNSS program. Once all of the IR pictures were taken for the upper portion of the suction surface, the IR camera was moved to the lower portion of the suction surface and both processes were repeated. For this process only five of the seven turbulence levels were tested because of the similarities between the small grid near, large grid, and aero combustor with spool configurations. After the data were taken, the power to the heaters was turned off and the system continued running until steady state was reached again. Afterwards the blower was turned off and the program was terminated for the day. 


\section{CHAPTER IV \\ EXPERIMENTAL RESULTS}

Experimental pressure and heat transfer measurements were acquired over the surface of the aft loaded vane. The measurements were taken at three different Reynolds numbers and seven different turbulence conditions. Three different pressure measurements were acquired which included an inlet pressure distribution, an exit pressure distribution, and a vane pressure distribution with static pressures acquired at the mid span of the vane. These experimental results were then compared to predictions based on a two dimensional periodic calculation done in FLUENT [34]. The heat transfer distributions were also taken at the mid span of the vane and are reported in terms of Stanton number based on the exit conditions of the cascade. Predictive comparisons were made by using STAN7 [35], a boundary layer code using an algrebraic turbulence model [36] and the Mayle transition model [37]. These predictive comparisons were used to help understand the heat transfer behavior over the surface of the vane. IR camera pictures of the suction surface were also taken, with the endwall heated and with no endwall heating. These measurements help our understanding of the effects of secondary flows on the vane heat transfer. These heat transfer distributions compare the effects of a heated starting length versus an unheated starting length on vane heat transfer distributions.

\section{Inlet and Exit Pressure Distributions}

The inlet and exit pressure distributions were taken to help determine the inlet uniformity and periodic nature of the exit flow through the cascade. These measurements helped us gain confidence that the cascade was constructed in a way which closely approximated a periodic 
flow over the vanes. The inlet pressure measurements were taken from 30 inlet pressure ports located one quarter chord from the leading edge plane of the vanes. The periodic nature of the inlet pressure distribution shows that the spacing is consistent in both the $\mathrm{x}$ and $\mathrm{y}$ - directions for all of the vanes as shown in figure 26. The spacing on the $\mathrm{x}$-axis is based on the distance the pressure port is from the leading edge of the instrumented vane. A negative $\mathrm{x}$ value means the pressure port is placed in the first or second full passage of the cascade, where a positive $\mathrm{x}$ value means that the pressure port is above the instrumented vane or in the third complete full passage. The y-axis is a dimensionless pressure value. This scaling makes it easier to compare the data from Reynolds number to another. The density and velocity values are based on the experimental conditions of the cascade.

$$
\mathrm{P}^{+}=P /\left(\rho * \frac{V_{E X I T}{ }^{2}}{2}\right)
$$

Equation 1

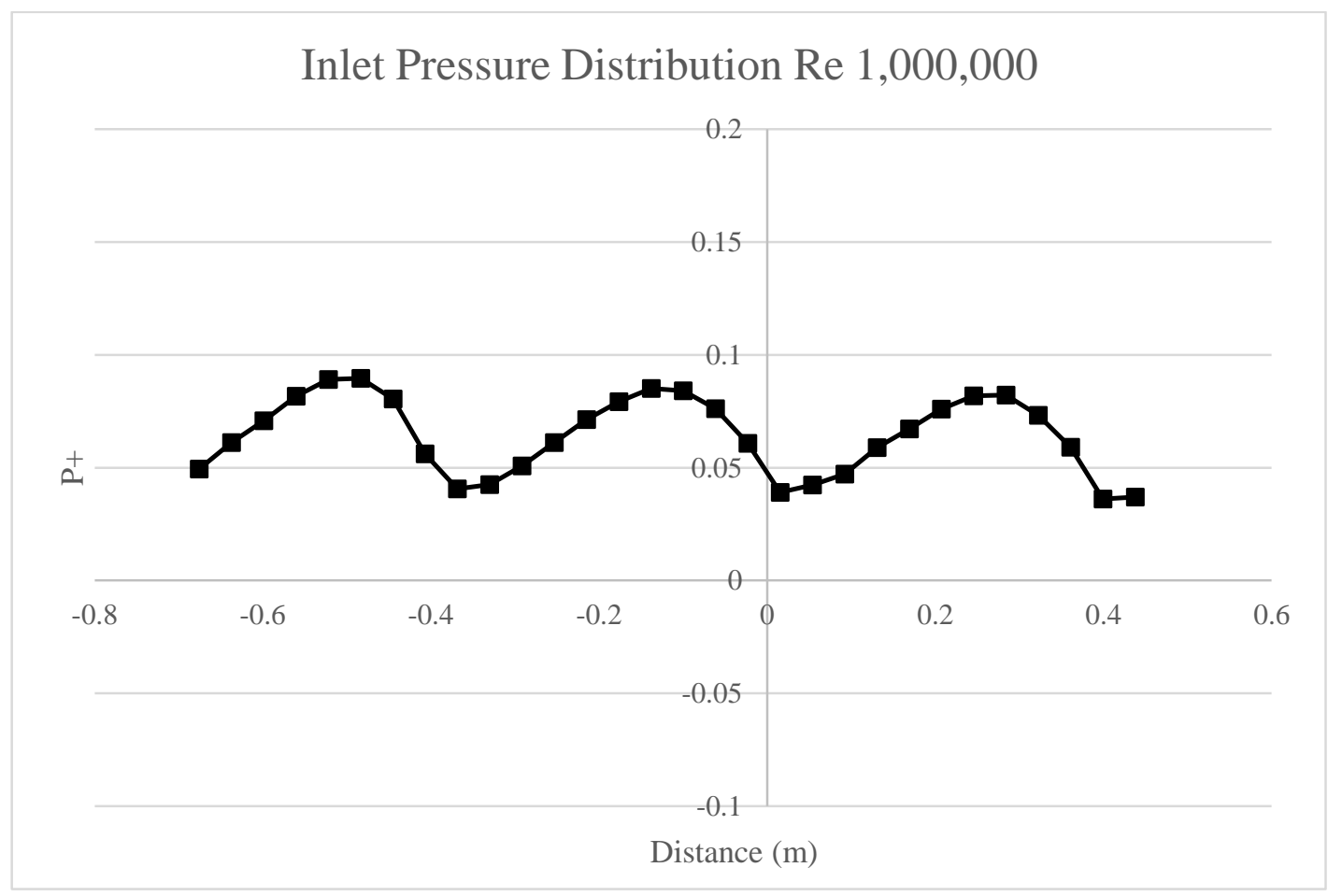

Figure 26. Low turbulence inlet pressure distribution Re 1,000,000 
The exit pressure distribution, similar to that of the inlet pressure distribution was based off of 30 pressure ports which were placed one quarter axial chord from the trailing edge plane of the vanes. This pressure distribution helped determine the periodicity of the exiting flow. It also provided confidence that the vanes were placed accurately and the tailboards were located at their proper positions. The exit pressure distribution can be seen in figure 27 . Both the inlet and exit distributions were only shown at the low turbulence level, due to the fact that a change in turbulence level has little effect on the static pressure readings. The $\mathrm{x}$-axis for this figure is based on the distance in meters the pressure port is from the first pressure port starting with the bottom pressure port in the first passage. The y-axis is the same dimensionless pressure value as the inlet pressure distribution.

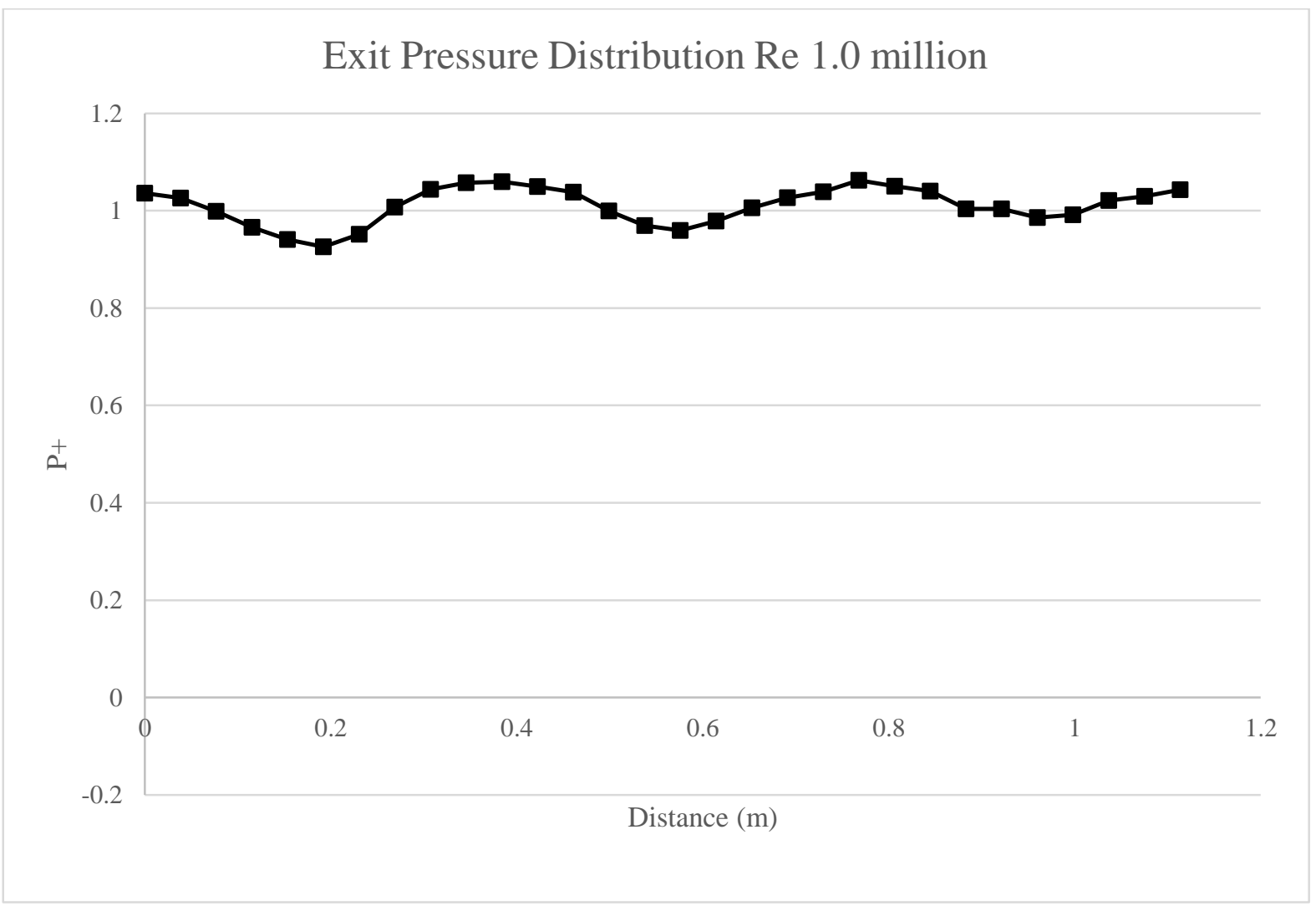

Figure 27. Low turbulence exit pressure distribution Re 1,000,000 


\section{Vane Pressure Distribution}

The mid span vane pressure distribution also helped in gaining confidence in the aerodynamics of the baseline cascade. The pressure vane was manufactured with 40 static pressure taps located around the surface of the vane with closer spacing around the leading edge of the vane. The vane was placed into the cascade as the third vane from the bottom. A mid span pressure measurement was acquired at all three Reynolds numbers, ranging from 500,000 to $2,000,000$, and at all seven turbulence levels. There was little to no change found in the pressure distributions around the vanes based on the turbulence level. Figure 28 shows the mid span pressure distribution of the small grid near configuration at a Reynolds number of $1,000,000$. These data are being compared to a 2-D calculation done in FLUENT [34] based upon the experimental conditions. The prediction used a reliable k-epsilon model [38] for turbulent closure.

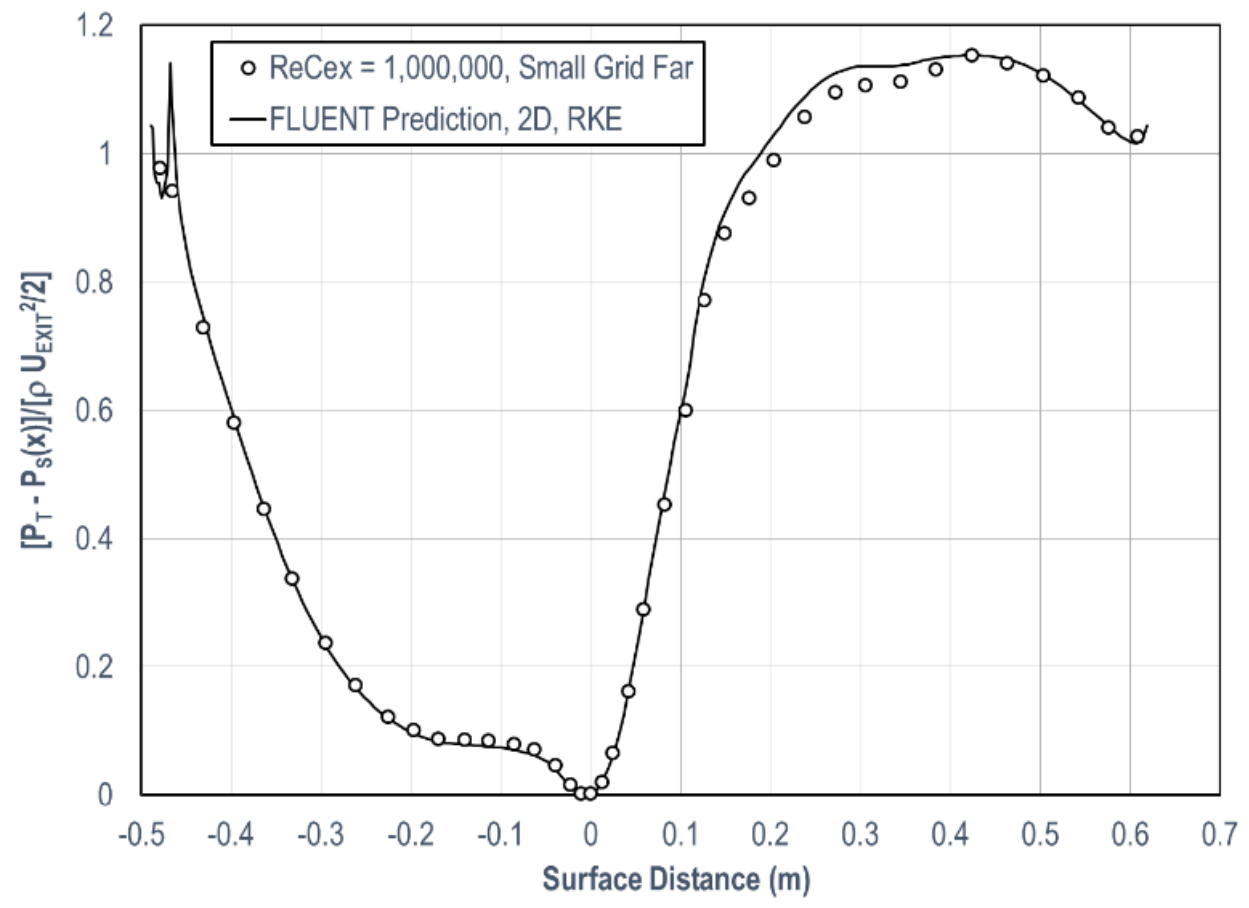

Figure 28. Small grid near vane pressure distribution with prediction $\operatorname{Re} 1,000,000$ 
The distances on the $\mathrm{x}$-axis for figure 28 were taken from the stagnation region $(0 \mathrm{~m})$ with a negative distance representing the pressure surface and a positive distance representing the suction surface. The comparison between the acquired data and the FLUENT prediction is generally very accurate and provides a high level of confidence in the experimental approach. There is one area on the suction surface where the model over predicts the experimental data. This difference could be due to a 2-dimensional model trying to predict a 3-dimensional flow. Alternately some small uncertainty in the placement of the tailboard could cause the difference when compared to the calculated positioning based on the streamlines of the flow and boundary layer growth. The pressure distribution also shows a minimum value at a distance close to twothirds along the suction surface, which typically means that the flow is accelerating over the majority of the surface of the vane. On the pressure surface the figure shows a strong acceleration through the stagnation region, and then a portion of steady flow as it travels downstream. The flow again starts to accelerate approximately thirty percent downstream from the stagnation region on the vane.

\section{Heat Transfer Measurements}

The heat transfer measurements were acquired over the surface of the vane using a constant heat flux technique. The heat transfer vane was constructed with 53 fine wired type kthermocouples along the surface of the vane to obtain a temperature distribution along the entire surface. A $0.023 \mathrm{~mm}$ Inconel foil was then wrapped around the vane to produce a constant heat flux for the heat transfer measurements. Copper bus bars were soldered into place on the foil to evenly distribute the supplied current. The copper bus bars fit into the machined grooves that were mentioned earlier and lay flat on the surface of the vane. When the heat transfer vane was exchanged for the pressure vane in the cascade the data could start being acquired. The cascade 
had to reach steady state with the unheated vane to acquire the recovery temperatures over the surface of the vane before the heat transfer data could be acquired. When this condition was recorded the constant heat flux foils could be turned on and again steady state had to be achieved. The driving force for the heat transfer measurements is the temperature difference between the heated vane surface and the adiabatic vane surface. If there is any difference in the inlet total temperatures between the heated and adiabatic runs it is used to help account for any differences between the runs.

$$
Q=I * V
$$

Equation 2

$$
Q^{\prime \prime}=Q / A
$$

Equation 3

The surface heat flux was determined from the voltage across the foil and the current through the foil measured using a precision shunt resistor that is connected in series with the foil. The net surface heat flux is assumed to be the dissipated heat flux minus the radiation loss based on the local surface to adiabatic wall temperature. The emissivity used in this calculation was previously determined to be 0.21 from past experiments. The heat transfer coefficient was calculated using the net surface heat flux divided by the temperature difference between the heated and unheated vanes taking the difference between the two inlet temperature probes.

$$
Q^{\prime \prime}{ }_{n e t}=Q^{\prime \prime}-\varepsilon_{m} * .0000000567\left(\left(T_{h}+273.15\right)^{4}-\left(T_{a}+273.15+T_{T h}-T_{T a}\right)^{4}\right)
$$

$$
h=Q^{\prime}{ }_{n e t} / T_{\text {diff }}
$$




$$
T_{\text {diff }}=T_{h}-T_{a}+\left(T_{T h}-T_{T a}\right)
$$

Equation 5

The heat transfer distributions have all been measured at the mid span of the vane and are reported in terms of the Stanton number based on the exit conditions.

$$
S t=h /\left(\rho * V_{E X I T} * C_{p}\right)
$$

Equation 6

Measurements have also been taken on the suction surface of the vane which assists in visualizing the effects of secondary flows on the suction surface heat transfer levels of the vane. The surface temperature distributions were acquired using a FLIR 500 thermal imaging camera, and the images have been calibrated using the thermocouples located on the surface of the vane.

\section{Vane Heat Transfer Distributions}

Vane heat transfer distributions were taken at the mid span of the vane and presented in the form of a Stanton number distribution. The Stanton number distributions are based upon the exit conditions of the cascade. The Reynolds numbers that the data are presented for are based upon the true chord length of the instrumented vane. In figure 29 the vane mid span heat transfer distribution based on Stanton number can be seen. The positive surface distance starts at the stagnation point and travels down the suction surface of the vane, while the negative surface distance is in reference to the pressure side of the vane. For the low turbulence setting the Stanton number distribution shows the general characteristic of laminar flow. As the turbulence levels start to increase the pressure side of the vane continues to show characteristics of laminar flow, but with turbulent augmentation in the areas where the flow is accelerating. The distributions also show a high Stanton number in the stagnation region of the vane, and an 
increase in this value with increasing turbulence levels. On the suction surface of the vane the Stanton values tend to slightly increase, then show a sudden drop in values as the air travels over the surface of the vane. As the turbulence levels increase the flow begins to transition and Stanton number distributions start varying from their laminar tendencies. By the time the highest two turbulence levels are reached the flow over the suction surface of the vane the flow completely transitions and a plateau in the recorded Stanton number occurs.

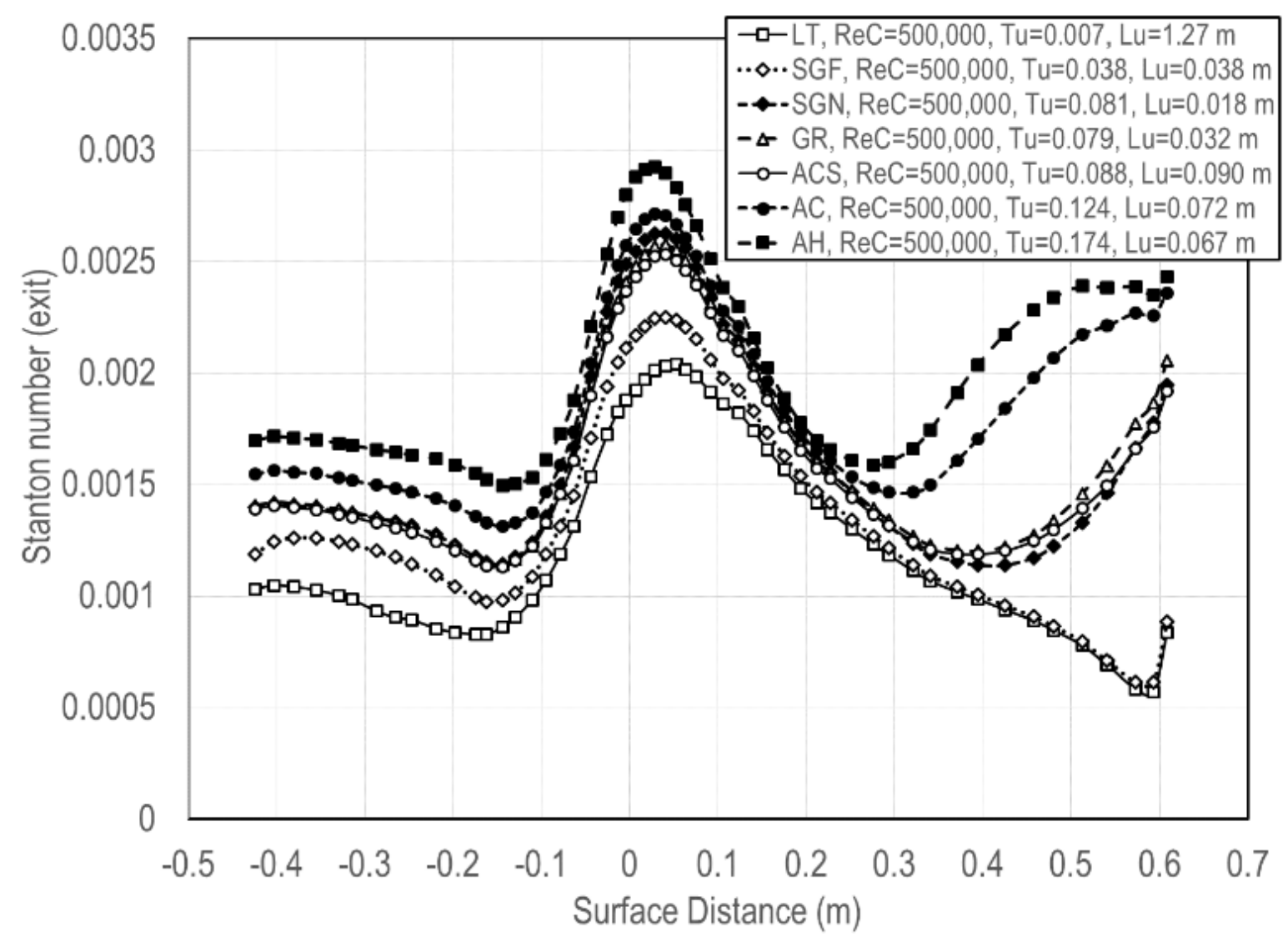

Figure 29. Vane Stanton number distributions with varying turbulence levels, $\operatorname{Re}_{c}=500,000$

The Stanton number distribution for Reynolds number of 1,000,000 can be seen in figure 30. This Stanton number distribution seems very similar to that of the 500,000 Reynolds number. However, the Stanton number levels have generally decreased. Also, when comparing the two figures, the pressure surface of the vane is very similar to the lower Reynolds number figure, but 
on the suction surface of the vane transition onset has moved forward towards the stagnation region. The flow is transitioning earlier and the peak Stanton value in the stagnation region has become lower than that on the turbulent section of the suction surface.

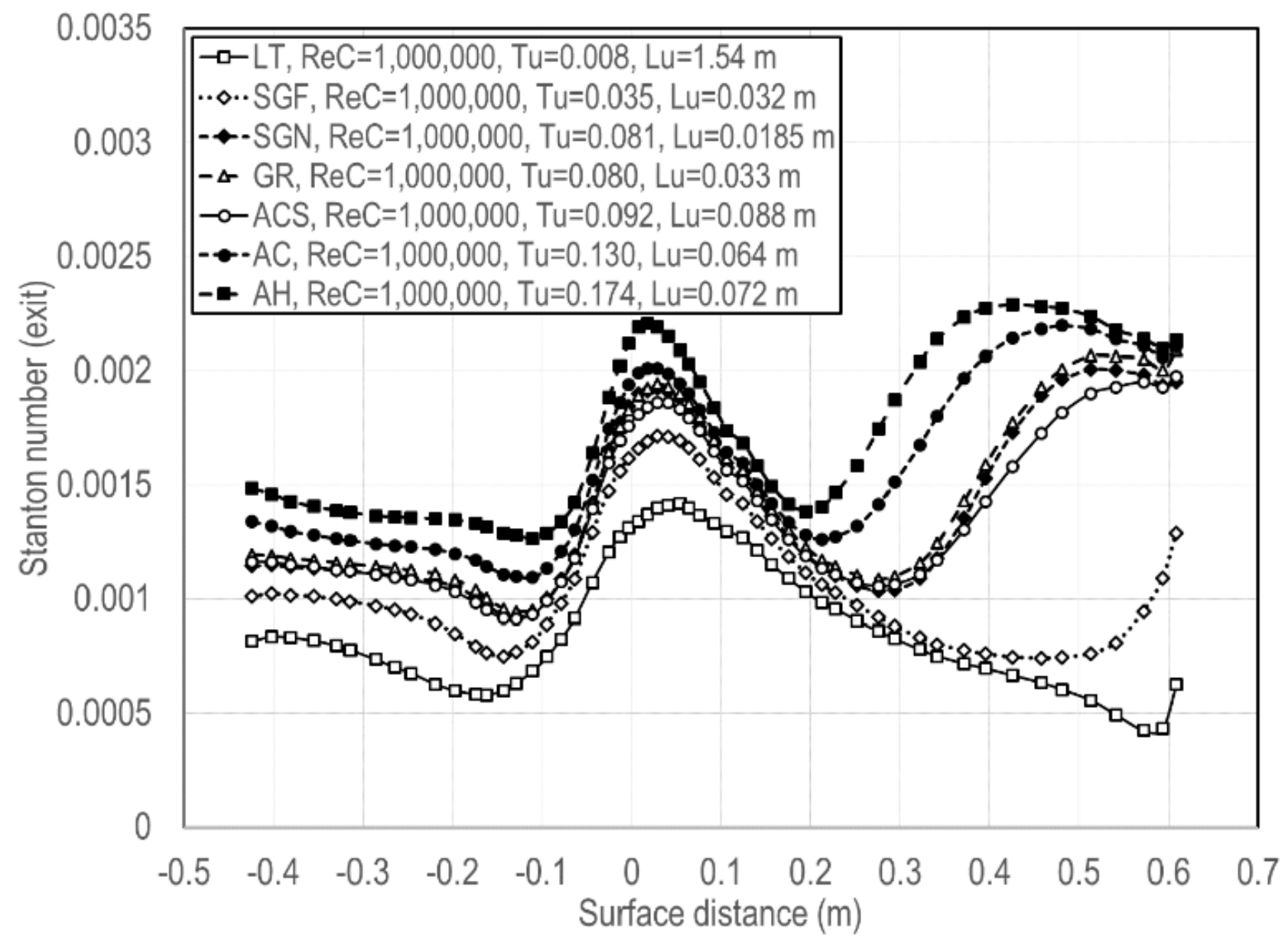

Figure 30. Vane Stanton number distributions with varying turbulence levels, $\operatorname{Re}_{c}=1,000,000$

The last Reynolds number tested for vane mid span heat transfer distributions was a Reynolds number of 2,000,000. The results can be seen in figure 31 . The Stanton number distributions show that the flow is now transitional on both the pressure side and the suction side of the vane. The transition is again occurring earlier than before on the suction side. Also, the trend of lower Stanton number values with increasing Reynolds number is continuing as expected. This trend of a lower peak Stanton number with increasing Reynolds number suggests 
that the leading edge heat transfer problem could become even more tractable with larger gas turbines. The other trend that began to show itself at a Reynolds number of 1,000,000 and continued to show itself at a Reynolds number of 2,000,000 is the transitioned flow is starting to have a higher Stanton value than the peak value at the stagnation region of the vane.

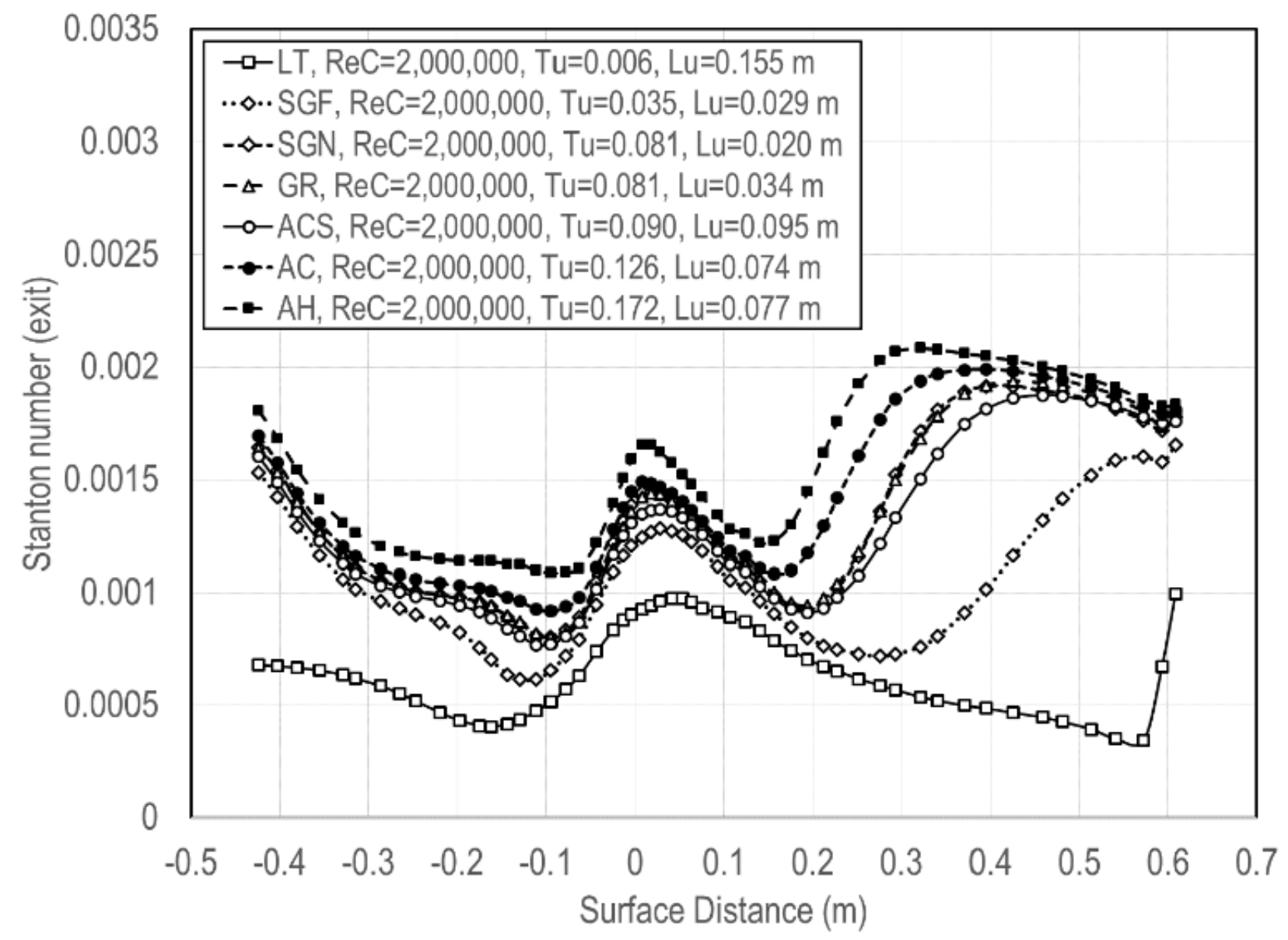

Figure 31. Vane Stanton number distributions with varying turbulence levels, $\operatorname{Re}_{c}=2,000,000$

Heat Transfer Augmentation and Transition

When looking at the heat transfer distributions it is helpful not only to look at the distributions based on Stanton number, but also present the distributions in terms of St-Sto. This $\mathrm{St}_{-} \mathrm{St}_{0}$ presentation shows both the heat transfer augmentation and the transition process. $\mathrm{St}_{-} \mathrm{St}_{0}$ presents the portion of the heat transfer increase from the low turbulence data to the higher turbulence conditions. The increase seen in the figures could be due to the augmentation process 
due to the increased turbulence level, or it could be due to the flow transitioning along the surface of the vane. If the difference is due to the augmentation process due to turbulence in the laminar region it is typically modeled as a thermal diffusivity plus an eddy diffusivity due to external turbulence times the mean temperature gradient.

$$
q^{\prime \prime}=\left(\alpha+\alpha_{T U}\right) \frac{d \bar{T}}{d y}
$$

Equation 7

If the increase in $\mathrm{St}_{-} \mathrm{St}_{0}$ is due to the flow starting to transition or transition onset the difference can be modeled by equation 8 .

$$
q^{\prime \prime}=\left[\alpha+(1-\gamma) * \alpha_{T U}+\gamma * \alpha_{T U R B}\right] \frac{d \bar{T}}{d y}
$$

Equation 8

In this equation $\gamma$ is the intermittency associated with the transition and $\alpha_{T U R B}$ is the eddy diffusivity associated with the intermittent turbulent boundary layer or passing spots. St-St $\mathrm{S}_{0}$ was plotted for all three different Reynolds numbers and figure 32 shows the difference between all six of the elevated turbulence levels when compared to the low turbulence condition at a Reynolds number of 500,000. The figure represents the difference in heat transfer for the different turbulence levels based on Stanton number with zero representing the stagnation region of the vane, with a positive surface distance being the suction surface and a negative surface distance representing the pressure surface. The peak at the stagnation region of the vane indicates the influence of turbulence on laminar heat transfer augmentation. The augmentation level is the highest in the stagnation region where the straining of turbulence by the flow intensifies the turbulence, producing more energetic fluctuations in the smallest scales. When the turbulence levels are smaller, like in the case of the small grid far condition, the turbulence can penetrate 
further into the boundary layer, due to its size, and enhance the mixing at the surface of the vane. This enhanced mixing at the surface of the vane increases the levels of heat transfer, therefore providing a difference between the lower levels of turbulence and the lowest level of turbulence. When the flow moves away from the stagnation region towards the pressure side of the vane the augmentation levels decrease, this could be due to the effects the convex curvature of the vane has on the turbulence level or a drop in the strain rate.

Downstream on the pressure surface of the vane a noticeable rise in the augmentation appears, but it appears to be earlier than the local acceleration in the flow. The data on the pressure surface does shows signs of transition occurring when the Reynolds number is equal to 500,000 at a surface distance of $-0.15 \mathrm{~m}$ from the stagnation region of the vane. However, due to the increased acceleration on the vane right after that area the transition process gets shut down and laminar flow continues towards the trailing edge of the pressure surface of the vane. On the suction surface of the vane the augmentation levels drop off considerably after the stagnation region. This drop could be due to the effects that the convex curvature of the vane has on the turbulence or the flow accelerating around the stagnation region of the vane. This drop continues to occur until transition begins. The trend of transition occurring on the suction surface of the vane only happens for the highest five levels of turbulence. When transition occurs there is a large increase in the augmentation levels. Also, as the turbulence levels increase transition tends to occur earlier and the augmentation levels increase. For the small grid far condition where there is no transition, there is little difference between the augmentation levels when compared to the low turbulence condition. 


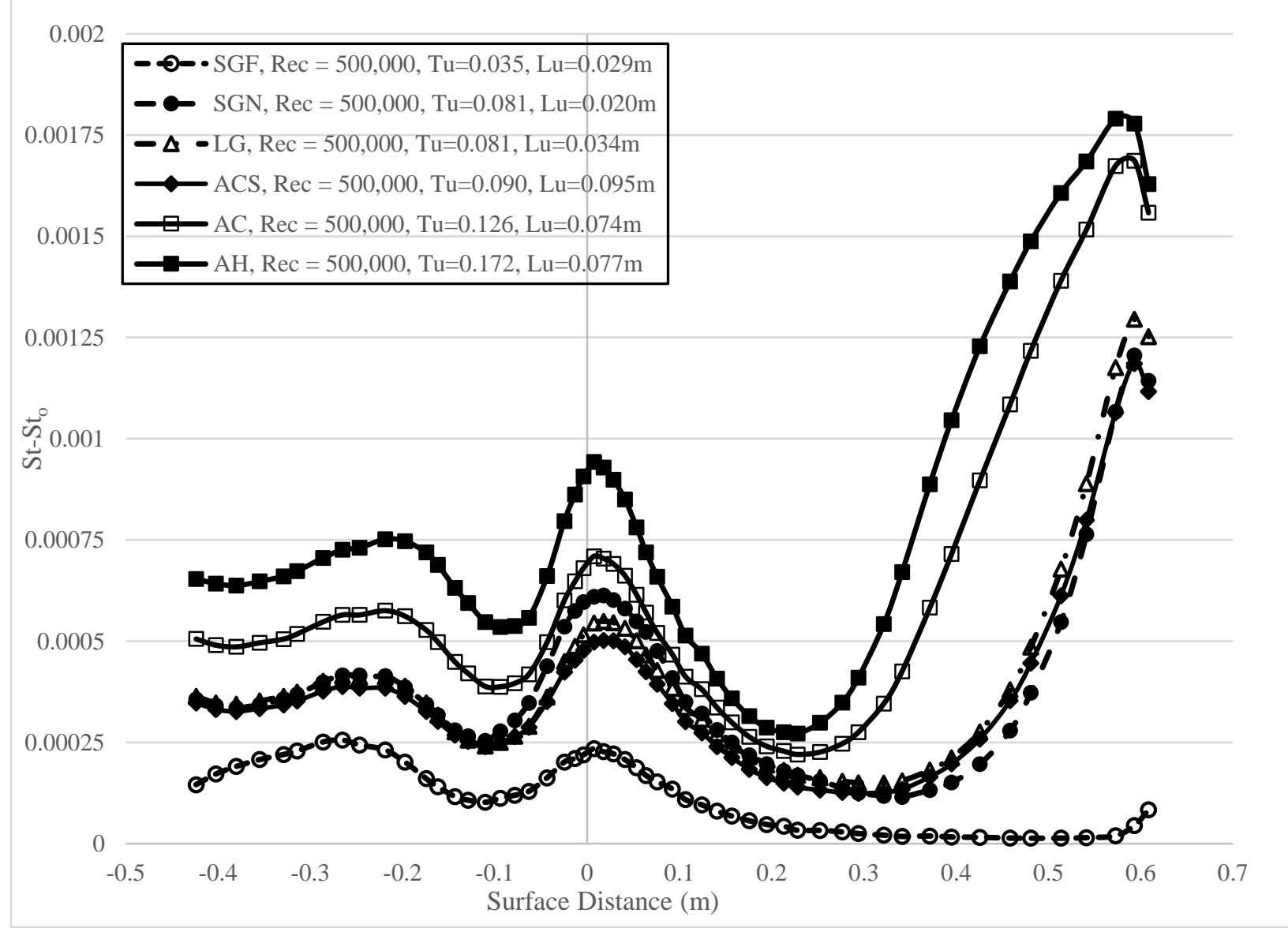

Figure 32. St-St 0 versus $X$ comparisons with varying turbulence levels at $\operatorname{Re}_{C}=500,000$

The Stanton number augmentation distribution, St-St 0 , for a Reynolds number of $1,000,000$ can be seen in figure 33 . The trends seen in this figure are very similar to that of the lower Reynolds number. There is a peak augmentation level at the stagnation region of the vane dropping off in both directions. On the pressure surface of the vane there happens to be a second peak value which is slightly lower than that at the stagnation region. This level of augmentation is due to the concave curvature of the vane which may produce Taylor-Goetler vortices or transition onset. On the suction side of the vane the augmentation continues to drop until transition occurs. For this Reynolds number transition occurs for all turbulence levels, and transition is moved upstream when compared to the lower Reynolds number. 


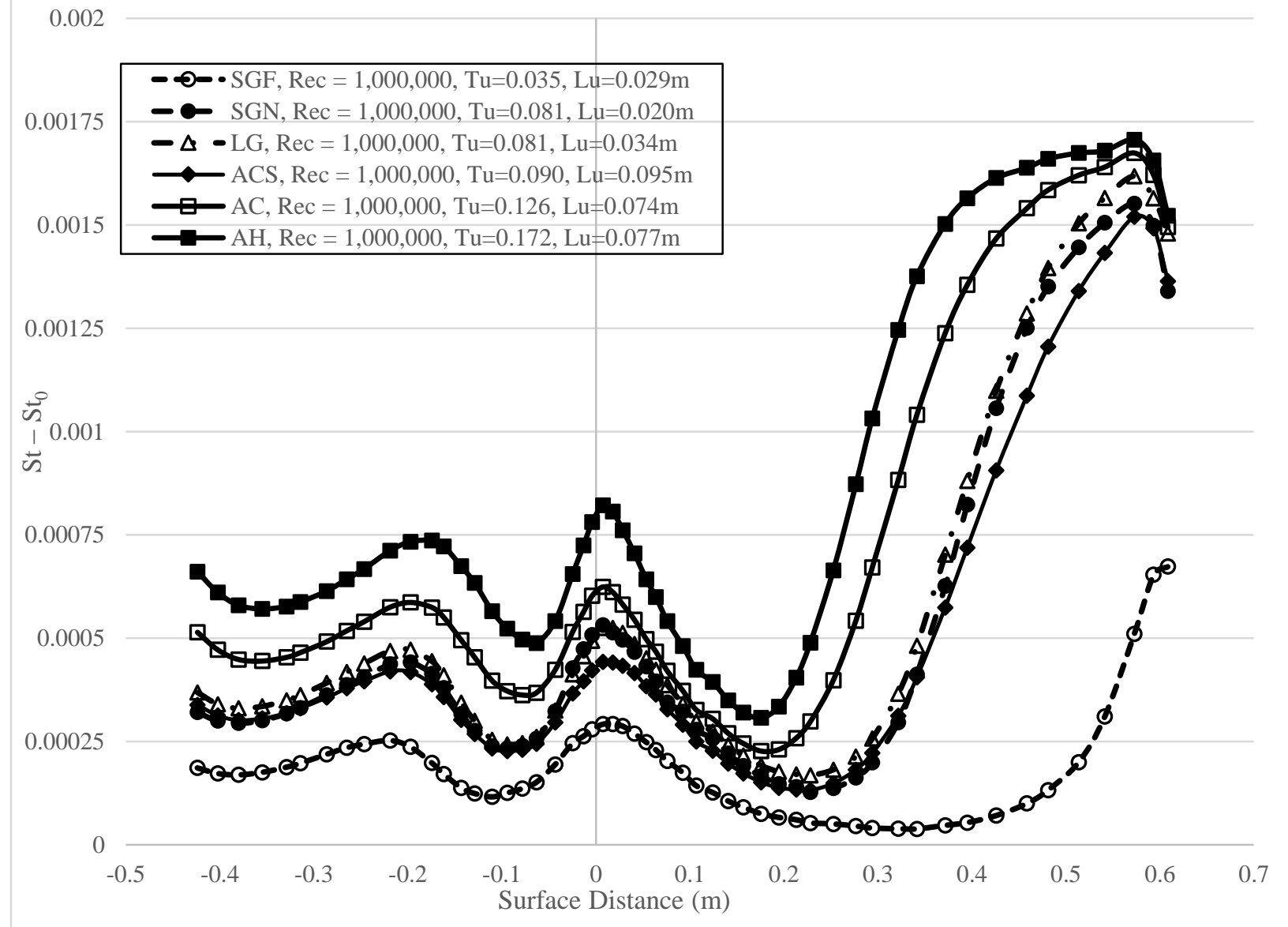

Figure 33. St-St $t_{0}$ versus $\mathrm{X}$ comparisons with varying turbulence levels at $\operatorname{Re}_{\mathrm{C}}=1,000,000$

Figure 34 show the Stanton number augmentation distribution for a Reynolds number of 2,000,000. Again the trends are very similar to those with the lower Reynolds numbers. Similar peaks are seen in the stagnation region, and the augmentation levels initially decrease on both the suction and pressure surfaces. This drop continues on the suction surface until transition, just like the lower Reynolds numbers, and transition continues to move further upstream. The difference is that for this Reynolds number the suction surface shows fully turbulent behavior for five of the six turbulence levels, instead of just transitional behavior. On the pressure surface the secondary peaks are now higher than the stagnation region peaks, which could indicate transitional behavior. Looking further upstream from the peaks it is clearly evident that the flow becomes transitional for all turbulence levels. 


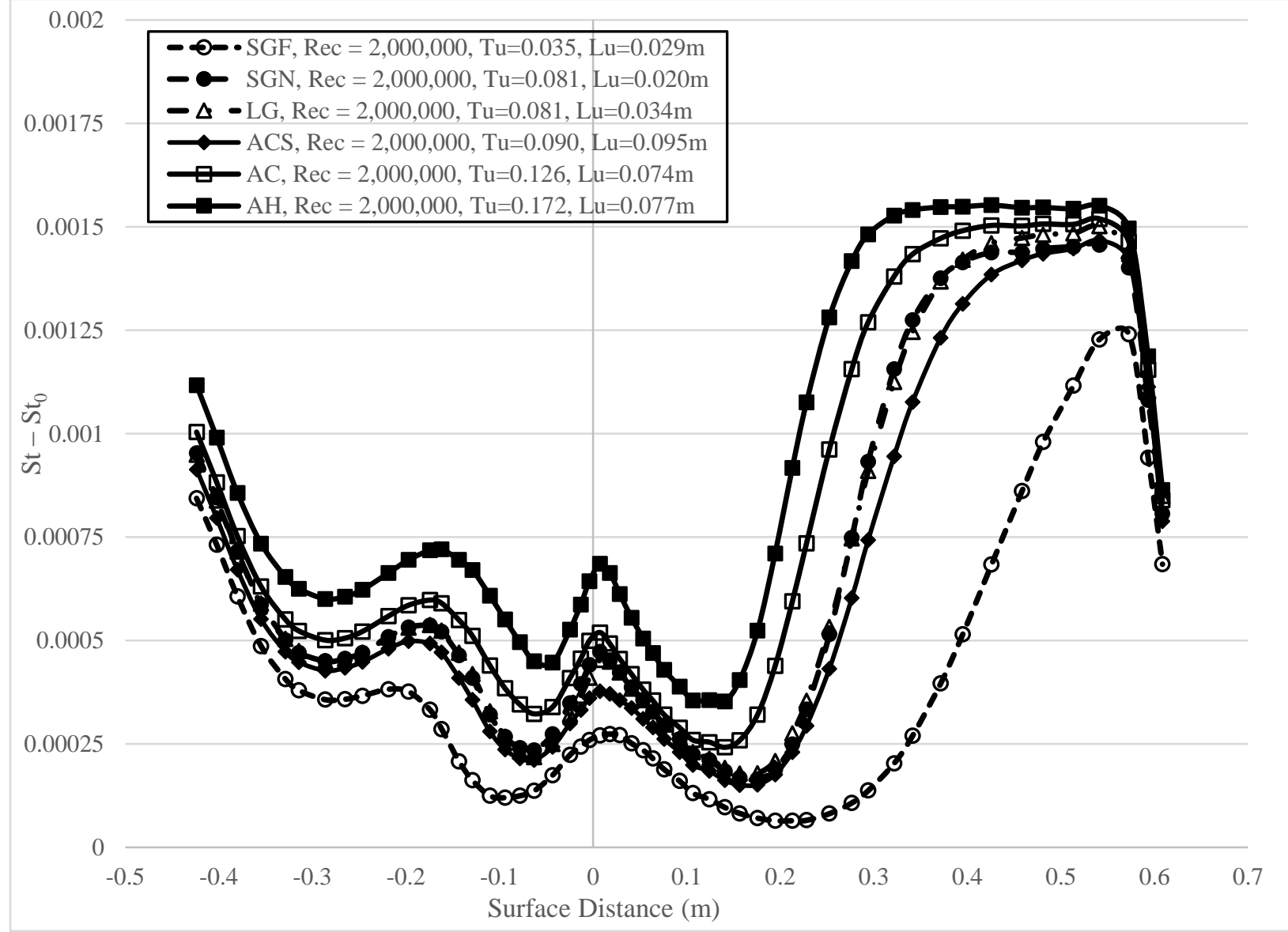

Figure 34. $\mathrm{St}-\mathrm{St}_{0}$ versus $\mathrm{X}$ comparisons with varying turbulence levels at $\operatorname{Re}_{\mathrm{C}}=2,000,000$

\section{Acceleration Profiles}

The streamwise acceleration profiles on the surface of the vane are presented in figure 35 .

They are presented to help understand the Stanton number distributions that were presented earlier. The acceleration of the stagnation region of a vane is typically very high and in this case broad, due to the large leading edge. However, the acceleration parameter K normally decreased towards the trailing edge. In this case on the pressure surface the $\mathrm{K}$ can be seen to drop, and then a period of significantly higher acceleration occurs before falling off again towards the trailing edge. On the suction surface the flow decelerates and then maintains a mild acceleration parameter for the remainder of the surface. 


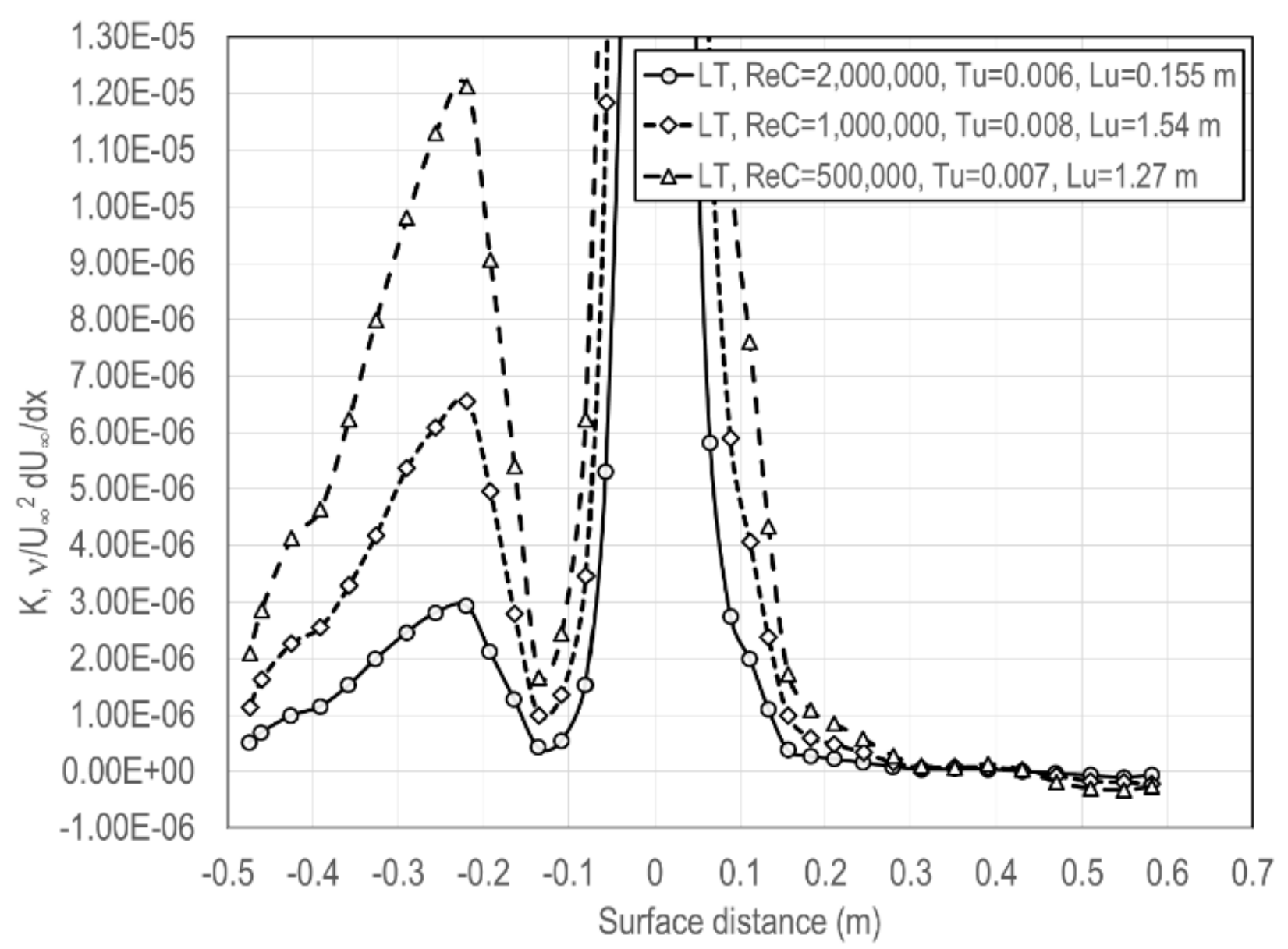

Figure 35. K distribution for vane pressure and suction surface

Heat Transfer Augmentation for Other Shapes

The Stanton number augmentation distributions in figures 32-34 present a complex

picture of how the flow is acting on the pressure surface of the vane. When compared to previous tests done at the University of North Dakota, the flow is not acting in a typical manner for laminar heat transfer on a pressure surface. Figure 36 shows the Stanton number distribution for the previous work done at the University of North Dakota on a conventional vane, Ames et al. [32]. The figure shows the Stanton number distribution at a Reynolds number of 1,000,000. 


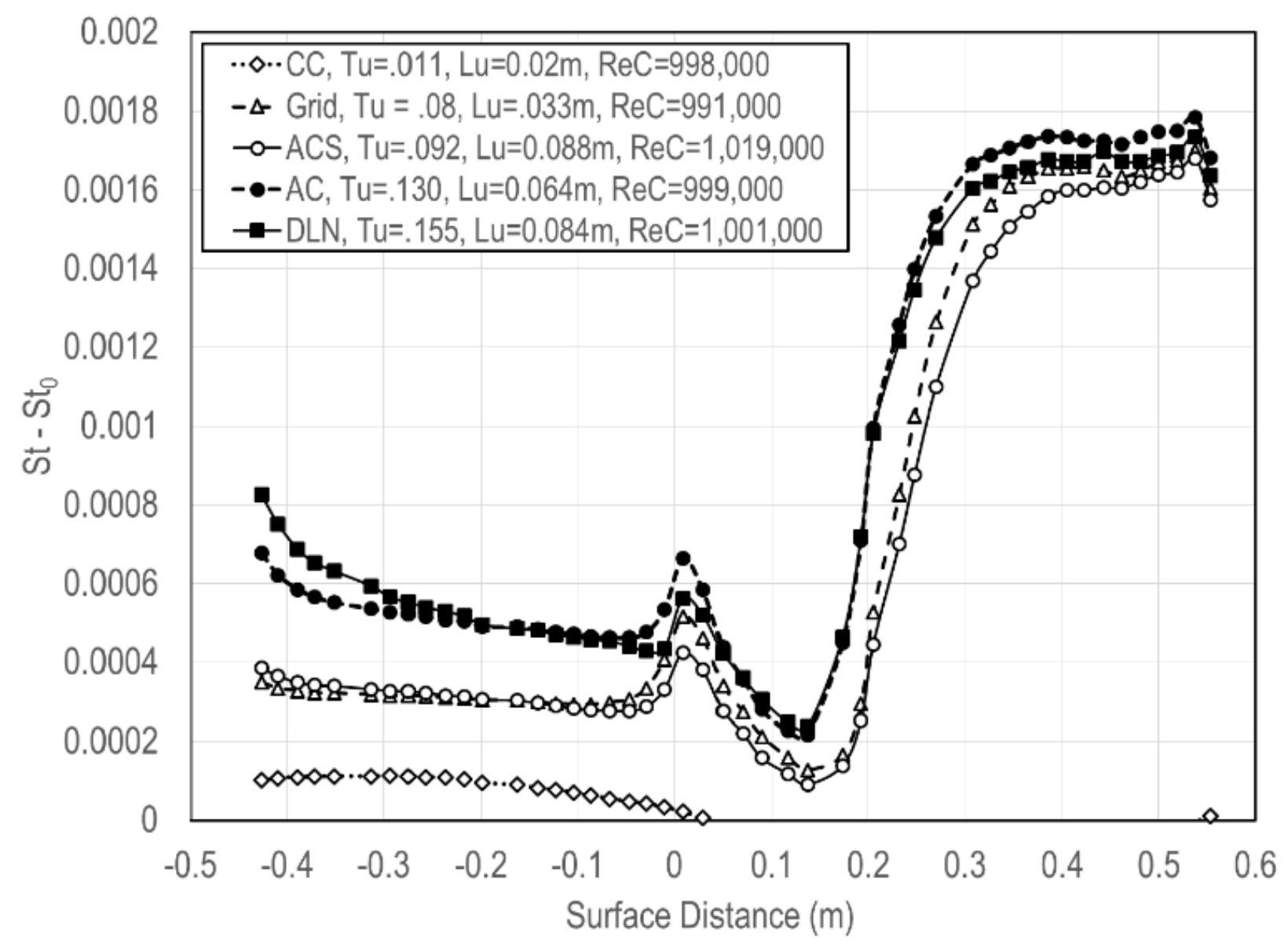

Figure 36. Comparison of $\mathrm{St}_{-} \mathrm{St}_{0}$ of a conventional vane [32]

This figure shows augmentation peaks at the stagnation area of the vane where the acceleration is the highest. Then the augmentation is relatively constant on the pressure surface, with no secondary peaks. This is where the vane with the larger leading edge differs from a conventional vane. As the flow continues on the pressure surface transitional behavior begins to show itself for the higher turbulence levels. On the suction surface the augmentation levels drop until the flow experiences transitional behavior. This behavior is consistent with the current vane design of a larger leading edge.

When comparing the current data collected to other shapes that also have been studied at the University of North Dakota. Ames and Kingery [11] provided Stanton number augmentation data on a large cylindrical test surface which can provide some interesting comparisons. The heat 
transfer augmentation data for the cylindrical test surface can be seen in figure 37. The data for the cylindrical test surface show the heat transfer augmentation levels in the leading edge region and the downstream area for a cylinder with a 4 inch $(10.16 \mathrm{~cm})$ diameter leading edge. In both cases the Stanton number calculations are based off of the exit conditions. Just like the vane data the augmentation values are very high in the stagnation region of the cylindrical leading edge test surface. Similar to the suction surface of the vane the heat transfer augmentation levels on the downstream surface drop off away from the stagnation region until transition occurs. The differences that can be seen in the figures are due to the differences between the exit to inlet velocity ratios which is the basis of Stanton number scaling. Vanes typically have an exit to inlet velocity ratio of around 3.5:1 where the exit to inlet velocity ratio for the cylindrical leading edge test surface is closer to $2: 1$.

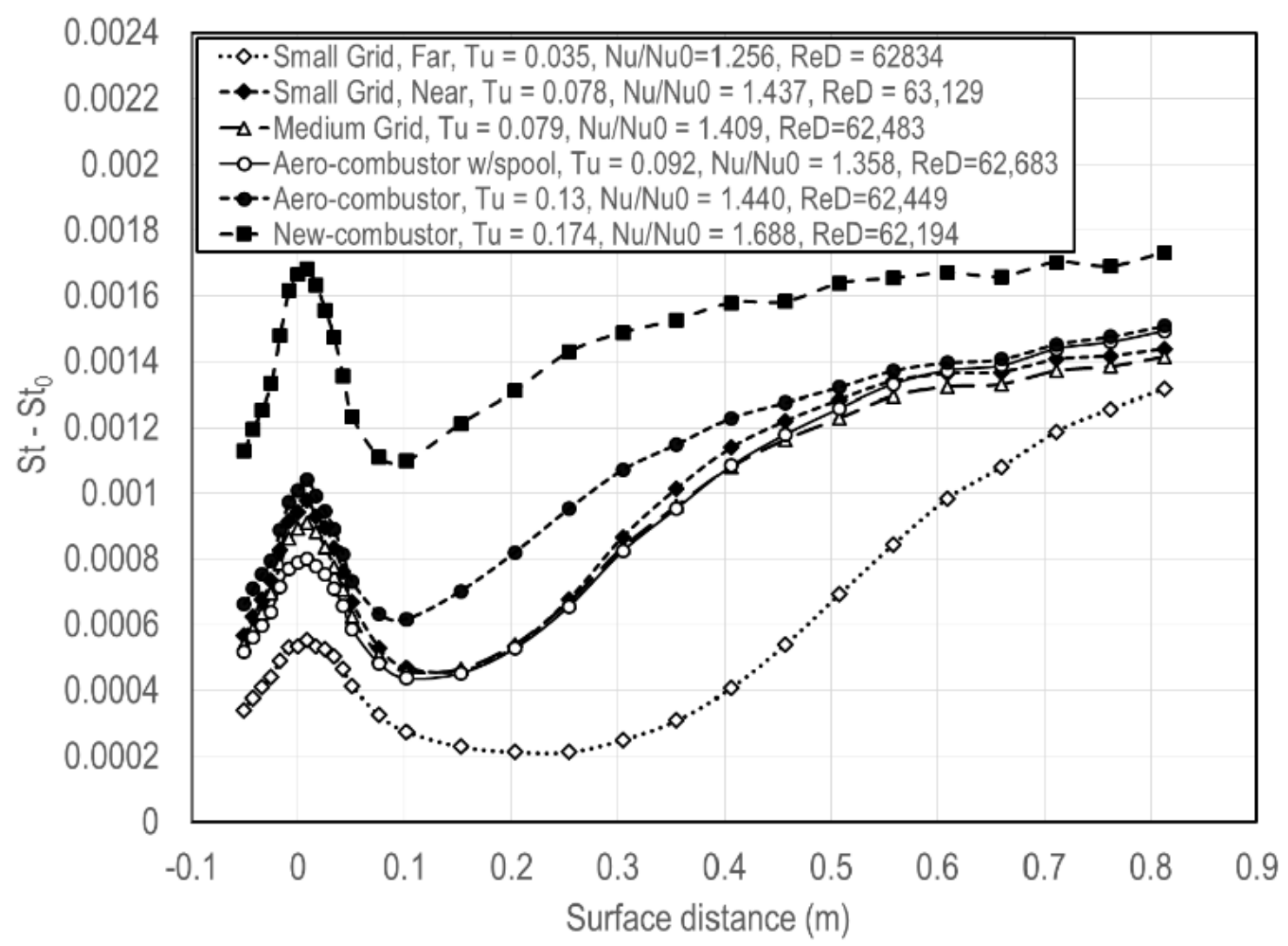

Figure 37. Comparison of $\mathrm{St}_{-} \mathrm{St}_{0}$ of a cylindrical leading edge test surface [11] 


\section{Stagnation Region Augmentation}

Stagnation region augmentation can be assessed in terms of the TRL parameter of Ames and Moffat [15]. The Stanton number augmentation ratio is potted versus the TRL parameter for the seven turbulence levels that were tested. A simple correlation based on earlier data [12] is plotted for a comparison.

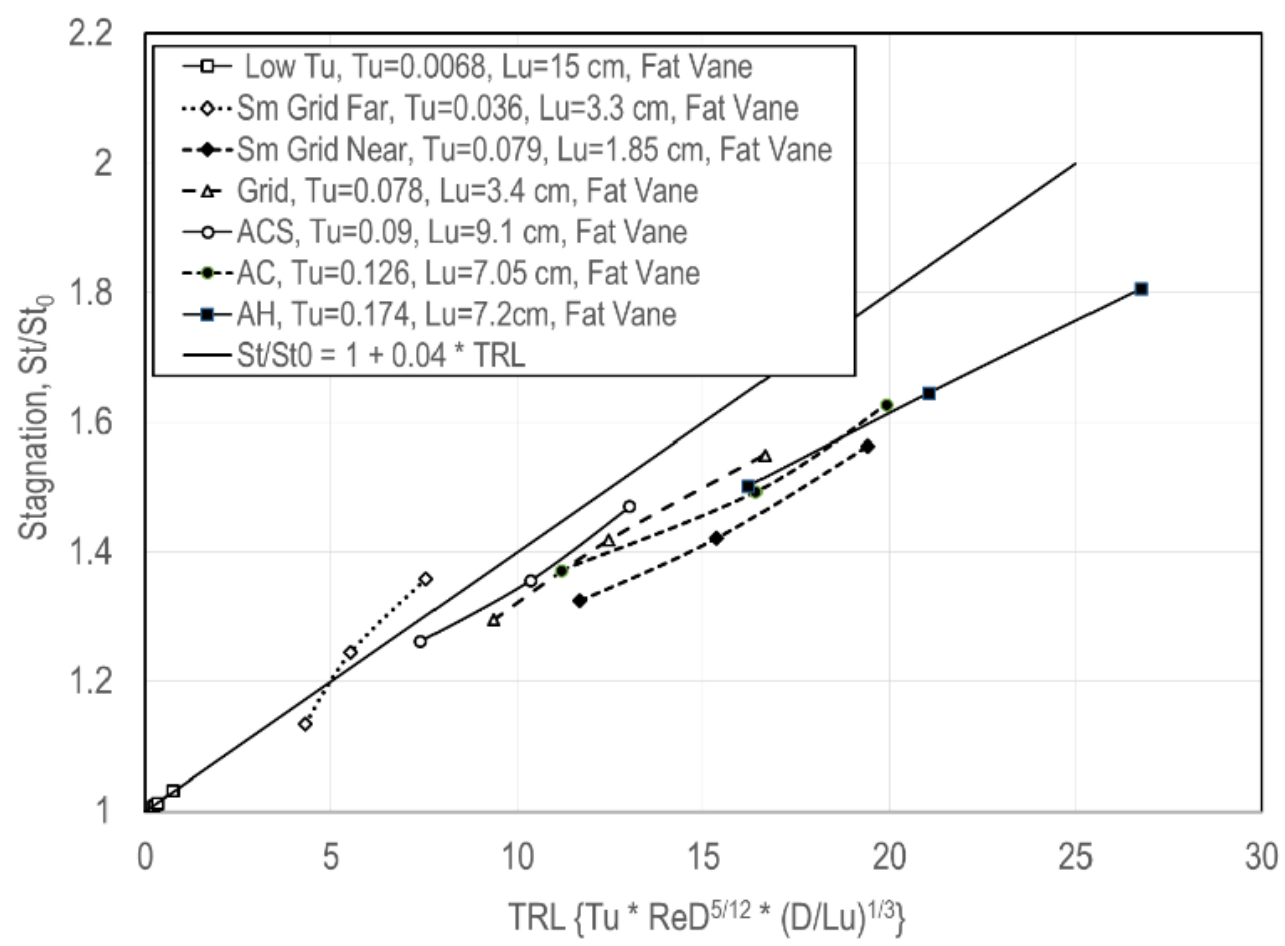

Figure 38. TRL comparison for vane stagnation region

When looking at the data in figure 38 it can be seen that the worst case scenario shows that the actual $\mathrm{St} / \mathrm{St}_{0}$ is approximately $13 \%$ below the predicted value. This discrepancy could be due to the larger leading edge of the vane. Due to the fact that the vane has a large leading edge it slows the approaching flow similar to the flow slowing down at the stagnation region of a cylindrical leading edge. This slowing velocity in the flow creates a longer convection time for the flow before it reaches the leading edge, which allows the turbulence levels to decay a greater amount than expected. This added decay in the turbulence levels can be attributed to the 
discrepancy in the value when comparing the value to the prediction. Assuming a relatively constant energy scale, the turbulence decay in the free stream can be estimated in the following equation.

$$
\frac{1}{T u}=\frac{1}{T u_{0}}+\frac{X-X_{0}}{2 \cdot L u}
$$

Equation 9

By comparing the relative turbulence augmentation levels using a pseudo time scale an indication of how much the turbulence decays due to the increased convection time should be evident. Smaller pseudo time scales should be more effected by decay. The actual to estimated Stanton number augmentation averaged for a turbulence condition is plotted versus a pseudo time scale can be seen in figure 39.

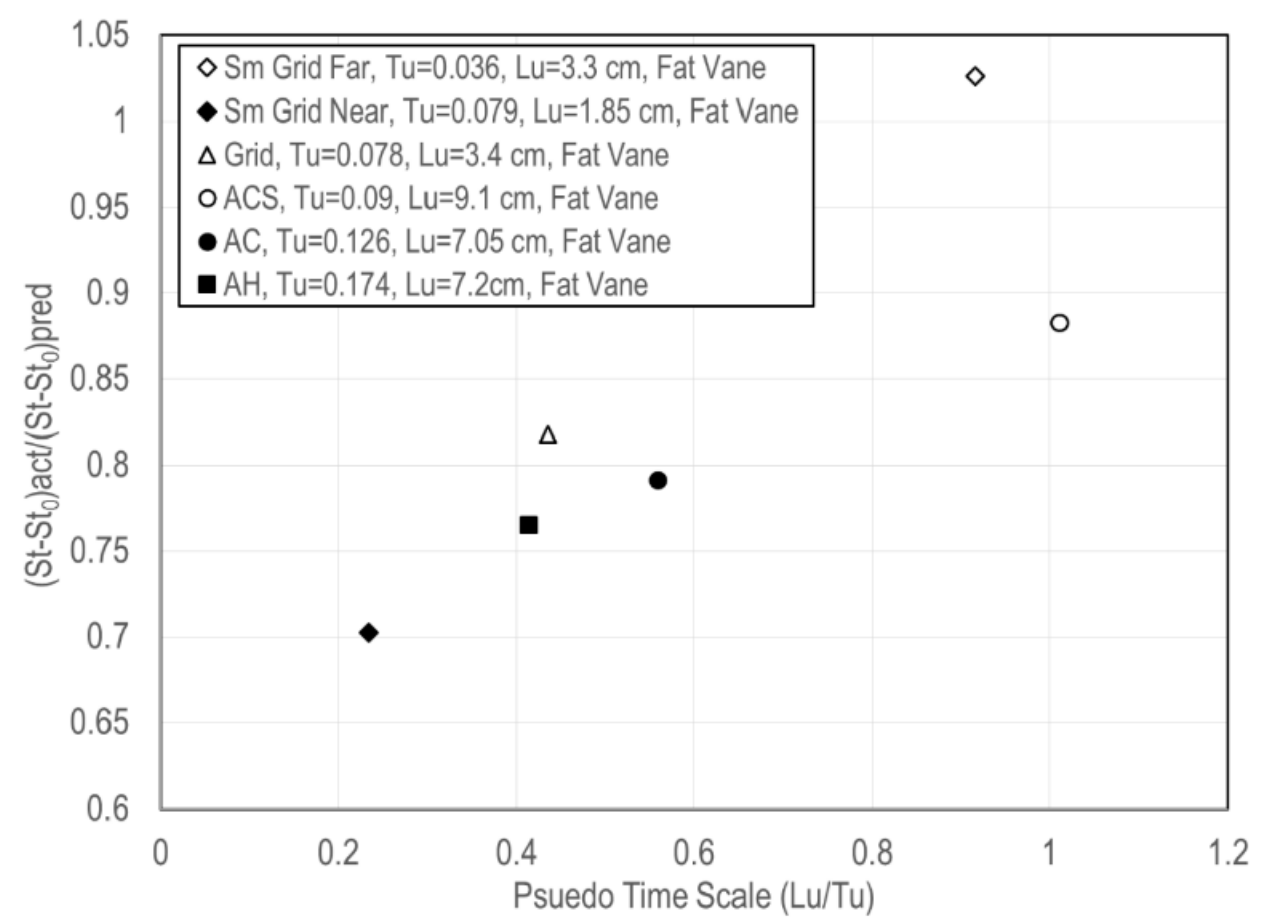

Figure 39. Actual/Predicted augmentation ratio versus pseudo time scale

This comparison generally shows an actual to predicted Stanton number ratio close to one as the time scale increases. For this case the discrepancies in the data help provide a reasonable 
explanation for the varying influence that the turbulence has on the vane. The turbulence levels generated by the mock aero-combustor are consistent with the turbulence levels and the relative scales that are expected to be generated in actual gas turbine combustion systems. Based on figure 38 and figure 39 the stagnation heat transfer data imply that the augmentation levels will be increasingly higher for larger gas turbines with higher inlet turbulence levels.

\section{Predictive Comparisons}

Predictive comparisons between the experimental data and the standard predictive tools used in this experiment can provide insight into the challenges faced in assessing the heat loads that are generated in gas turbine engines. Predictive comparisons were done based on the Stanton number at all seven turbulence levels that were tested during the experimental procedure and at all three Reynolds numbers. The predictions were made using a finite difference boundary layer code, STAN 7 [35]. This boundary layer code uses the algebraic turbulence model of Ames et al. [36] to account for the influence of the external turbulence on mixing and the transition model of Mayle [37].

Figure 40 shows the experimental results compared to the predictions made at an exit Reynolds number of 500,000. The figure only shows five different predictions due to the fact that the effects of turbulence on the small grid near, large grid, and aero combustor with spool are all very similar. This means that only one of these three different configurations was chosen to represent these very similar heat transfer levels, the large grid configuration. When analyzing the comparisons between the predictions and the experimental results the low turbulence condition shows a very close resemblance to the predictions done. There is a slight over prediction in the stagnation region of the vane, but on the suction surface they resemble each other well. However, on the pressure surface the prediction seem to under predict the 
experimental data towards the trailing edge. Based on this data this could be due to the formation of Taylor Gortler vortices. Overall the relative closeness of the prediction and experimental results at the low turbulence level provides confidence in the experimental approach. As the turbulence levels start to increase the predictive model tends to under predict the heat transfer levels in the stagnation region of the vane. Generally, this seems to be the case when using this boundary layer code at elevated levels of turbulence. The ATM does not account for the intensification of turbulence in the stagnation region which leads to this under prediction. On the pressure surface the trend of under predicting the heat transfer levels continues for all of the six higher turbulence levels. This again can be attributed to the formation of Taylor Gortler vortices.

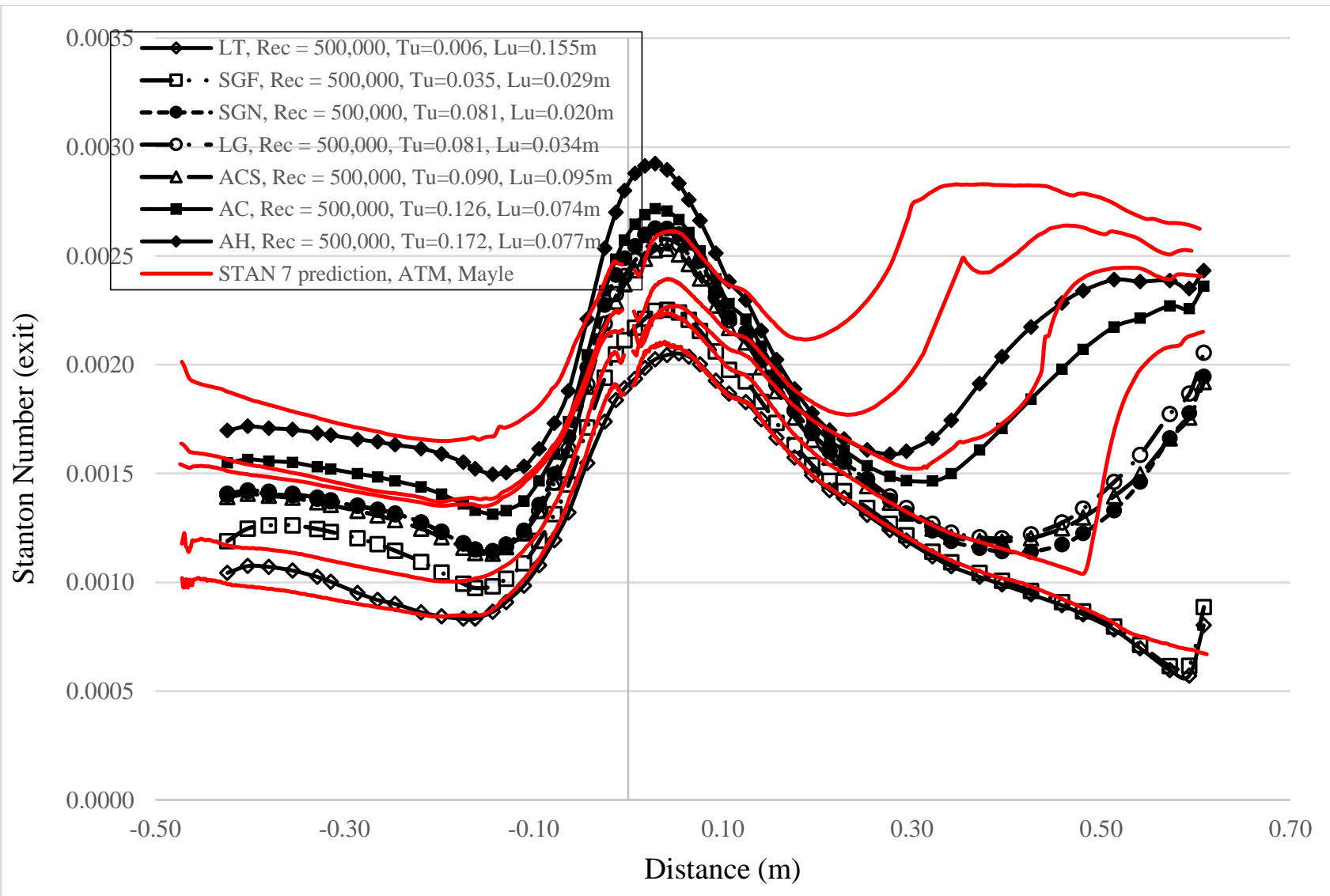

Figure 40. Predictions for vane Stanton number with varying turbulence, $\operatorname{Re}_{c}=500,000$ 
The Stanton augmentation generally shows a systematic reduction downstream on the suction surface. This drop in heat transfer augmentation is consistent with the drop in the acceleration of the flow around the vane, but it may also be due to the convex curvature on the suction surface of the vane. The vane was designed with an aft-loaded suction surface to help delay transition until further down the vane keeping the flow laminar for as long as possible, therefore helping reduce heat transfer levels in turbulent boundary layers [38]. This could be a possibility why the data in figure 40 shows reduction in turbulent augmentation developing on the suction surface of the vane. Another possibility could be the decay of turbulence on the suction surface of the vane is causing the reduction of turbulent augmentation of laminar flow. When comparing the heat transfer levels to the predictive models at the stagnation region of the vane a trend of significant under prediction begins. This carries over when the algebraic turbulence model begins its prediction for the suction surface of the vane. At the beginning of the suction surface of the vane the predictions show a slight under prediction, then they show early transition. This trend of predicting early transition only becomes greater as the turbulence levels increase. This could be because the Mayle transitional model used does not account for convex curvature effects when predicting transition. Also, it should be noted that the minor spikes in heat transfer on the suction surface when the predictions are in the transitional region of flow can be contributed to a spike in the models spot production as the spline fit to the experimentally determined velocity distribution predicts a short and mild period of an adverse pressure gradient. Also,

The predictions and Stanton number distributions for a Reynolds number of 1,000,000 can be seen in figure 41. Qualitatively the predictions are very similar to those shown at a Reynolds number of 500,000. The pressure surface shows an under predicted heat transfer level, 
but overall it is fairly accurate. However, as the turbulence levels start to increase the predictions show the flow starting to transition where the data shows no trend of transitional flow. There is still a significant under prediction in the stagnation region of the vane, with it getting worse as the turbulence levels increase. On the suction surface, the models still predict early transition on all flows which transition. For this Reynolds number the only flow that doesn't transition is for the low turbulence level.

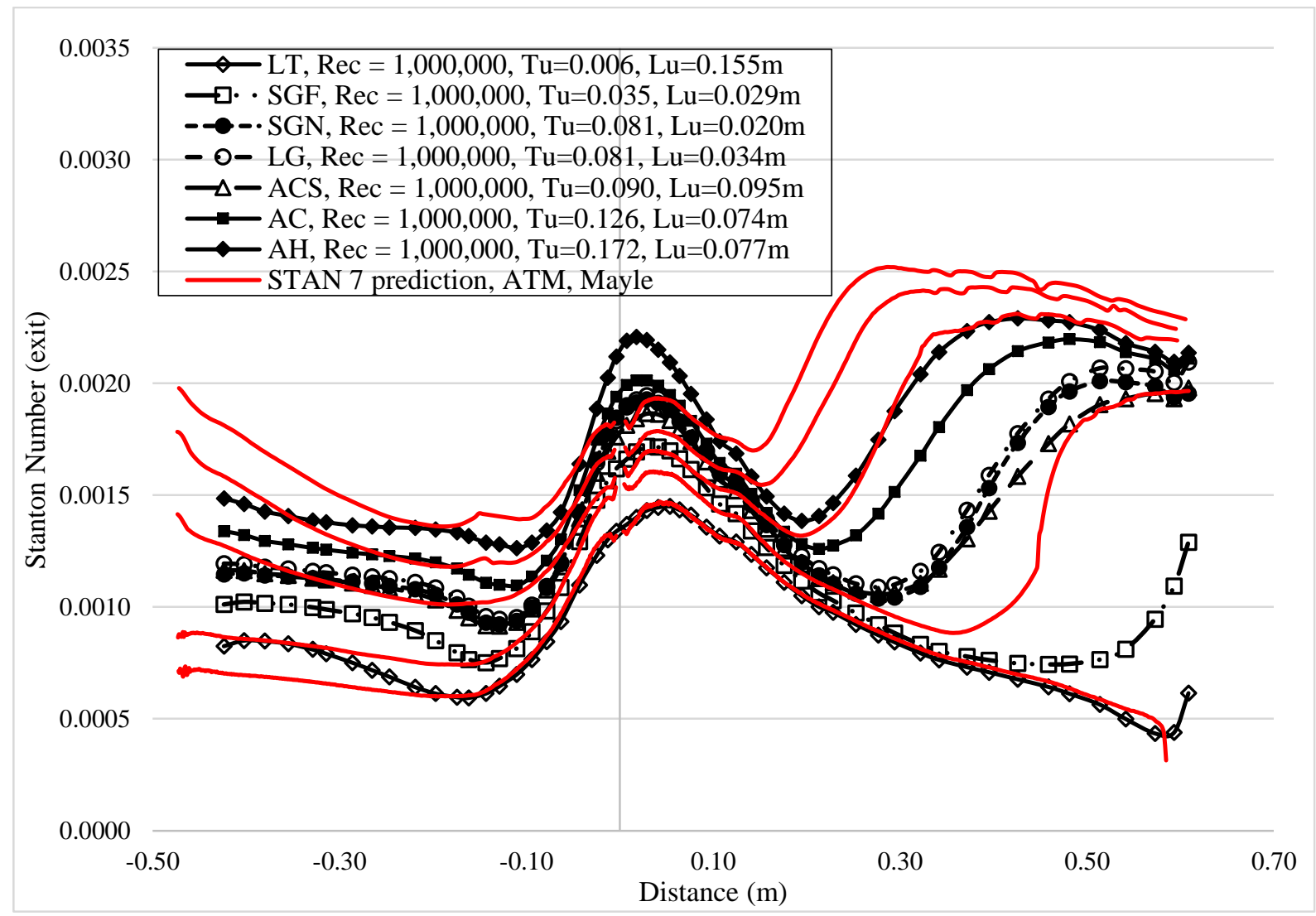

Figure 41. Predictions for vane Stanton number with varying turbulence, $\operatorname{Re}_{\mathrm{c}}=1,000,000$

Figure 42 shows the heat transfer distributions based on Stanton number and the predictions made for a Reynolds number of 2,000,000. When comparing this figure to the lower two Reynolds numbers the same trends continue to be seen. On the pressure side the predictions tend to under predict when the flow is laminar, which only seems to be the case for the low turbulence condition. The prediction under predicts the heat transfer collected for the small grid 
far condition as well. However, this case the data collected show that the flow is transitional where the prediction doesn't show the flow transitioning until the very end of the vane. On the suction surface all of the turbulence conditions except for the low turbulence condition the predictions tend to show early transition. The same trend of significantly under predicting the heat transfer levels at the stagnation region of the vane for the higher levels of turbulence continues.

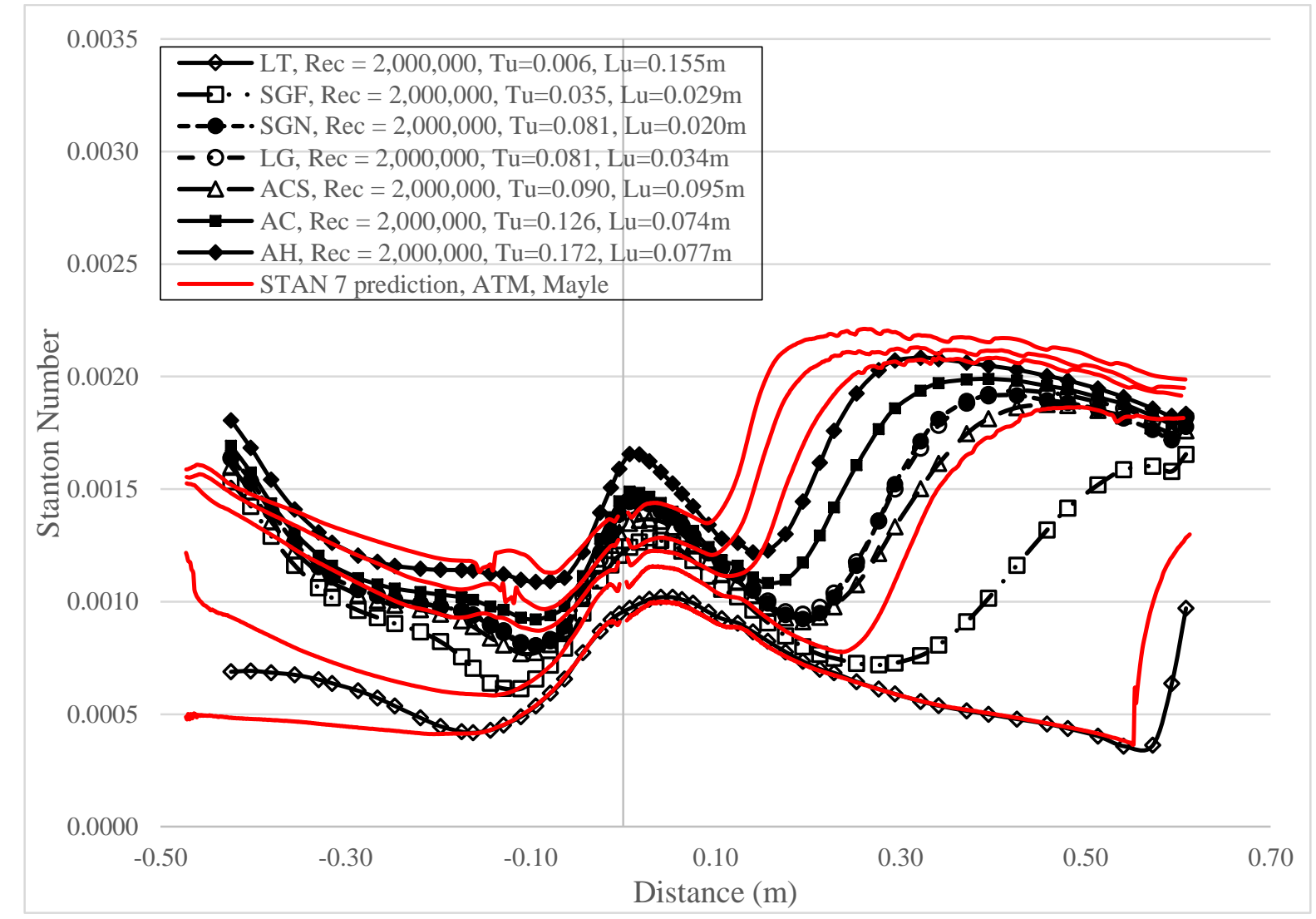

Figure 42. Predictions for vane Stanton number with varying turbulence, $\operatorname{Re}_{c}=2,000,000$

Overall these predictions tend to be on the conservative side for all Reynolds numbers, but a few trends do appear between them. Under predicting the heat transfer levels at the stagnation region, predicting early transition on the suction surface, and slightly under predicting the heat transfer on the pressure surface are the main trends. The area that seems to need the 
most improvement is at the stagnation region of the vane where there seems to be some issues in the eddy diffusivity models when predicting the heat transfer.

\section{Secondary Flows}

Secondary flows seem to be caused by streamwise vorticity due to viscous shear on the endwalls. The physics of secondary flows is highly complex and consists of several systems. Sieverding [39] identified the three main vortices existing in a blade passage including the horseshoe vortex, the passage vortex, and the counter vortex. The horseshoe vortex consists of a pressure side leg and a suction side leg. Figure 43 shows a model that Langston [40] developed and it suggests that separation occurs and a horseshoe or leading edge vortex is formed. This vortex travels through the passage with an opposite sense of rotation. The passage vortex merges with the endwall and entrains fluid from the mainstream of the passage. Where the counter vortex is much smaller and generally remains at the area where the suction surface of the vane and the endwall meet.

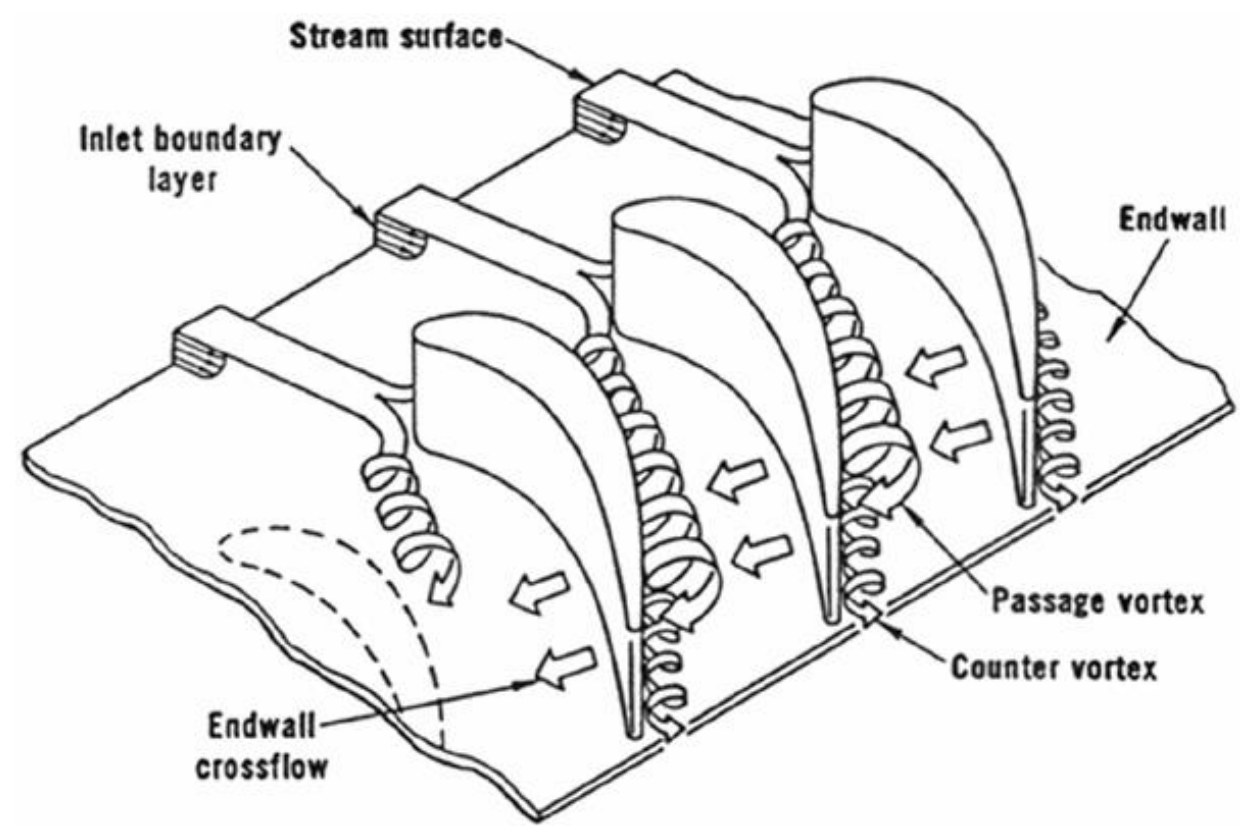

Figure 43. Langston et al. model depicting endwall vortices 
The main secondary flow that was visualized from the IR camera tests that were performed on the suction surface of the vane was the passage vortex flow coming off of the endwall and onto the surface of the vane. As the flow traveled through the passage of the cascade the passage vortex was able to travel along the vane moving upwards on the surface towards the trailing edge. This moved a secondary loss core over the surface of the vane which can be seen in the three dimensional suction surface Stanton number figures.

\section{Effects of Endwall Heating on Vane Heat Transfer}

Tests were made with the endwall heat turned on, to simulate the heated starting length, and endwall heat turned off, to simulate an unheated starting length to see the effects of a heated starting length on the vane heat transfer. Stanton number heat transfer distributions were calculated on the suction surface of the vane to help compare the differences between the unheated and heated starting length. The tests were performed at all three of the Reynolds numbers stated earlier, but at five of the seven turbulence levels. Since the data of the small grid near, large grid, and aero-combustor with spool were so similar it was decided that only testing one of these three turbulence levels would give a good representation for all three of them. IR camera pictures were taken at two different ports, a lower port which captured the suction surface of the vane 6.5-14.5 inches downstream from the leading edge and an upper port which captured the final 6.5 inches of the trailing edge on the suction surface. The slight rises that can be seen in the suction surface Stanton number figures are the gold dots that were painted onto the surface of the vane to help determine the location. The dots on the vane were painted in a 2 x 2 inch grid, starting $3 / 4$ of an inch from the trailing edge and $1 / 2$ an inch from the edge of the vane. Figure 44 and 45 show the suction surface Stanton number distributions for the small grid far turbulence condition at a Reynolds number of 1,000,000. The $\mathrm{x}$-axis on the figures represents the 
spanwise direction on the vane where the pixel value of 1 represents the edge of the vane and 240 represents the area near the midspan of the vane. The y-axis on the figures represents the streamwise direction of the flow with the flow entering the figure at the pixel number of 320 and exiting the figure at 1 . The figures show a spike in heat transfer levels on the left of the figure, which is the interface between the vane and the unheated endwall. This is expected due to the unheated starting length phenomenon. When the cold air from the wind tunnel meets the heated vane the thermal boundary layer is very thin, causing this high rate of heat transfer, which can be seen for all figures with an unheated starting length.

In figure 44, the lower portion of the suction surface for the small grid far turbulence condition, the flow remains laminar for the duration of the figure. The Stanton number remains low until the temperature difference between the heated vane and the endwall become near and the Stanton number begins to rise, dramatically. As the flow travels to the top portion of the suction surface, figure 45, the laminar flow just starts to show a trend of becoming transitional. The spike in heat transfer levels at the interface between the heated vane and unheated endwall is caused by the temperature difference between the two. This spike in heat transfer at the interface of the unheated endwall and the heated vane would make cooling the vane internally very difficult if this occurred in an actural engine. Figure 44 also shows a rise in heat transfer due to the unheated starting length phenomenon at approximately 1 inch from the edge of the vane and that grows to approximately 2.5 inches from the surface of the vane by the time the flow reaches the trailing edge, seen in figure 45 . This growth can be attributed to the upward movement passage vortex on the surface of the vane. 


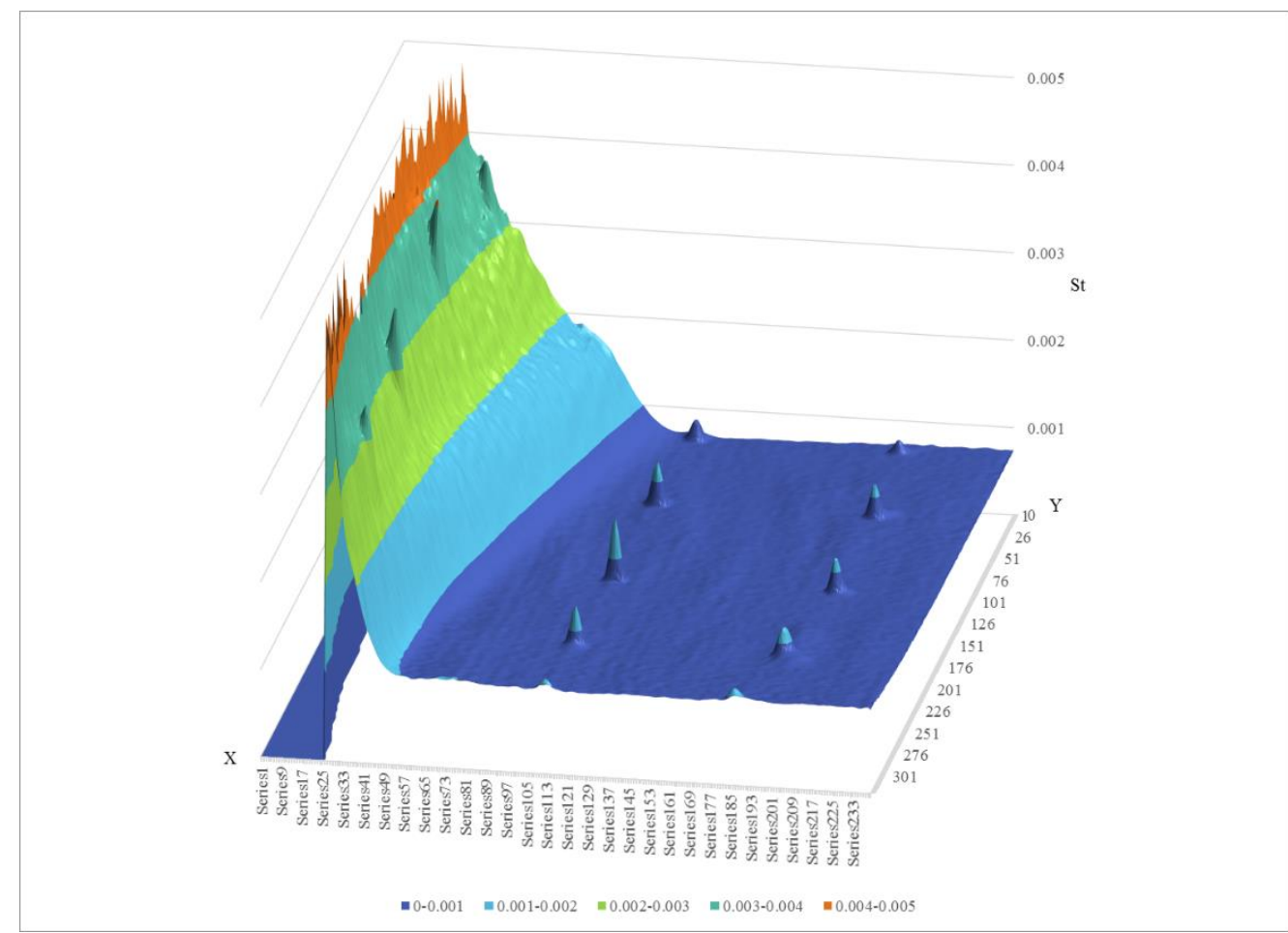

Figure 44. Suction surface Stanton number chart $\mathrm{Re}=1,000,000, \mathrm{Tu}=0.035$, unheated starting length, bottom

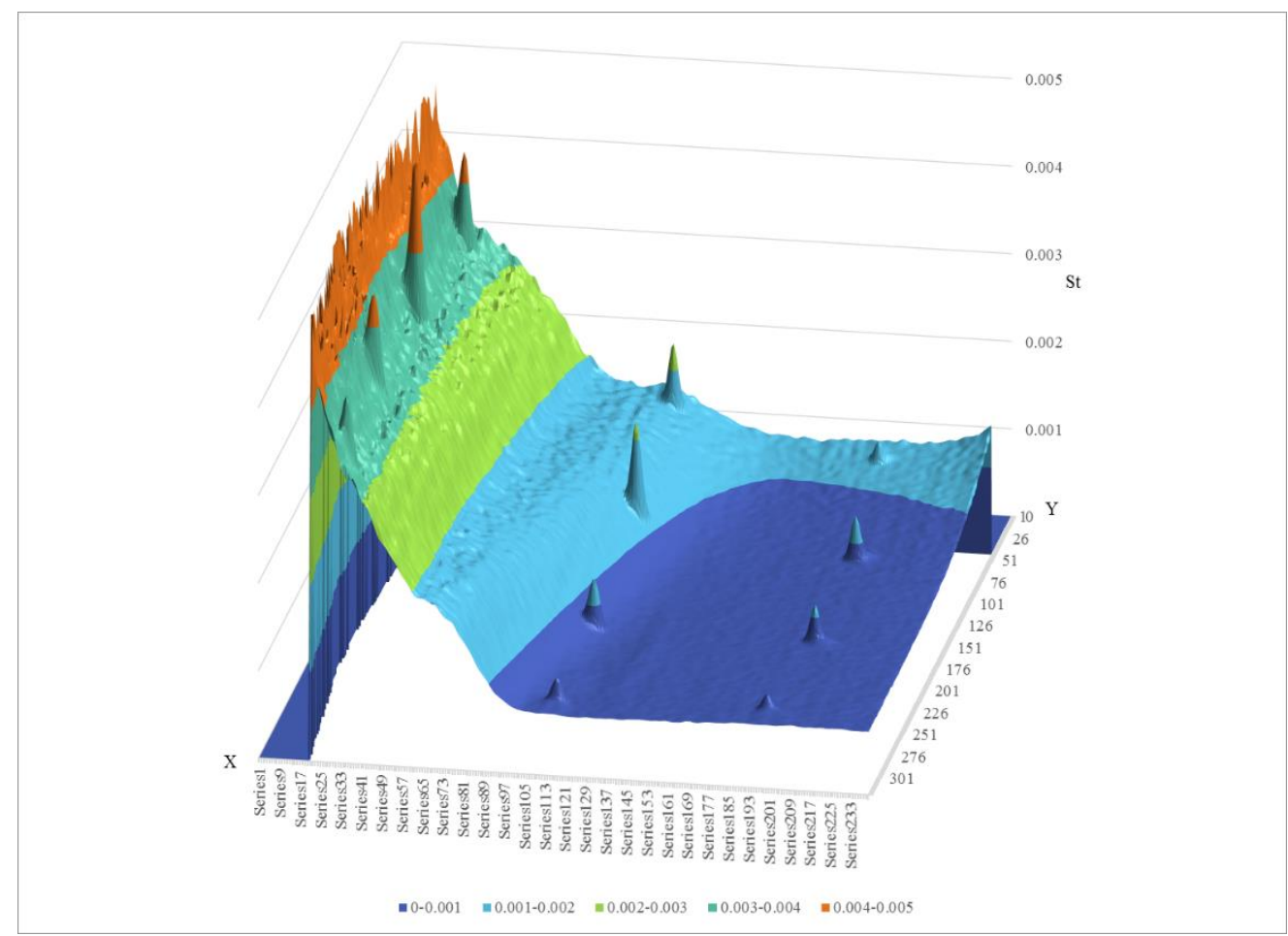

Figure 45. Suction surface Stanton number chart $\mathrm{Re}=1,000,000, \mathrm{Tu}=0.035$, unheated starting length, top 
As the turbulence level increases certain trends begin to show themselves in figures 46 and 47. The flow which was laminar in the lower section of the small grid far turbulence condition, figure 44 , is now becoming transitional in the lower section of the large grid turbulence condition, figure 46 . When comparing the higher turbulence condition to the lower tubulence condition the Stanton number is higher at the midspan of the vane, but lower at the edge of the vane. This may be attributed to the more coherent passage vortex system that is created by the lower turbulenc levels. As the flow travels to the upper portion of the suction surface, figure 47 , the flow has become fully turbulent. The transitional behavior has made the Stanton number higher, but the Stanton number remains at a fairly constant value of 0.00175 for the turbulent flow. Also, a peak in the Stanton number can be seen near the midspan of the vane, the third row of gold dots on the vane. This can be attributed to a higher roughness on the surface of the vane due to the painted surface. The increase in roughness is causing the flow to transition earler causing this spike in heat transfer near the midspan of the vane. 


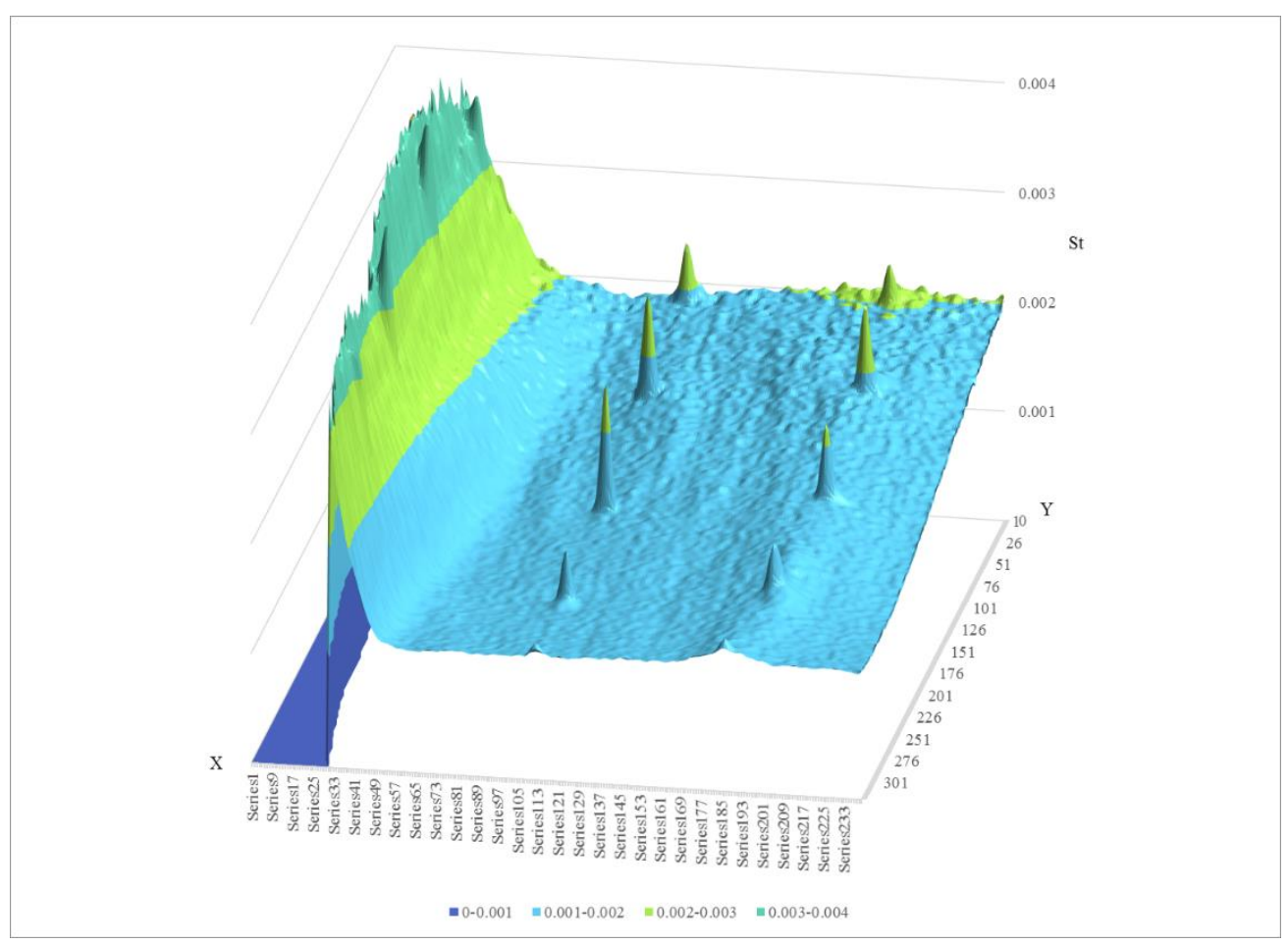

Figure 46. Suction surface Stanton number chart $\mathrm{Re}=1,000,000, \mathrm{Tu}=0.081$, unheated starting length, bottom

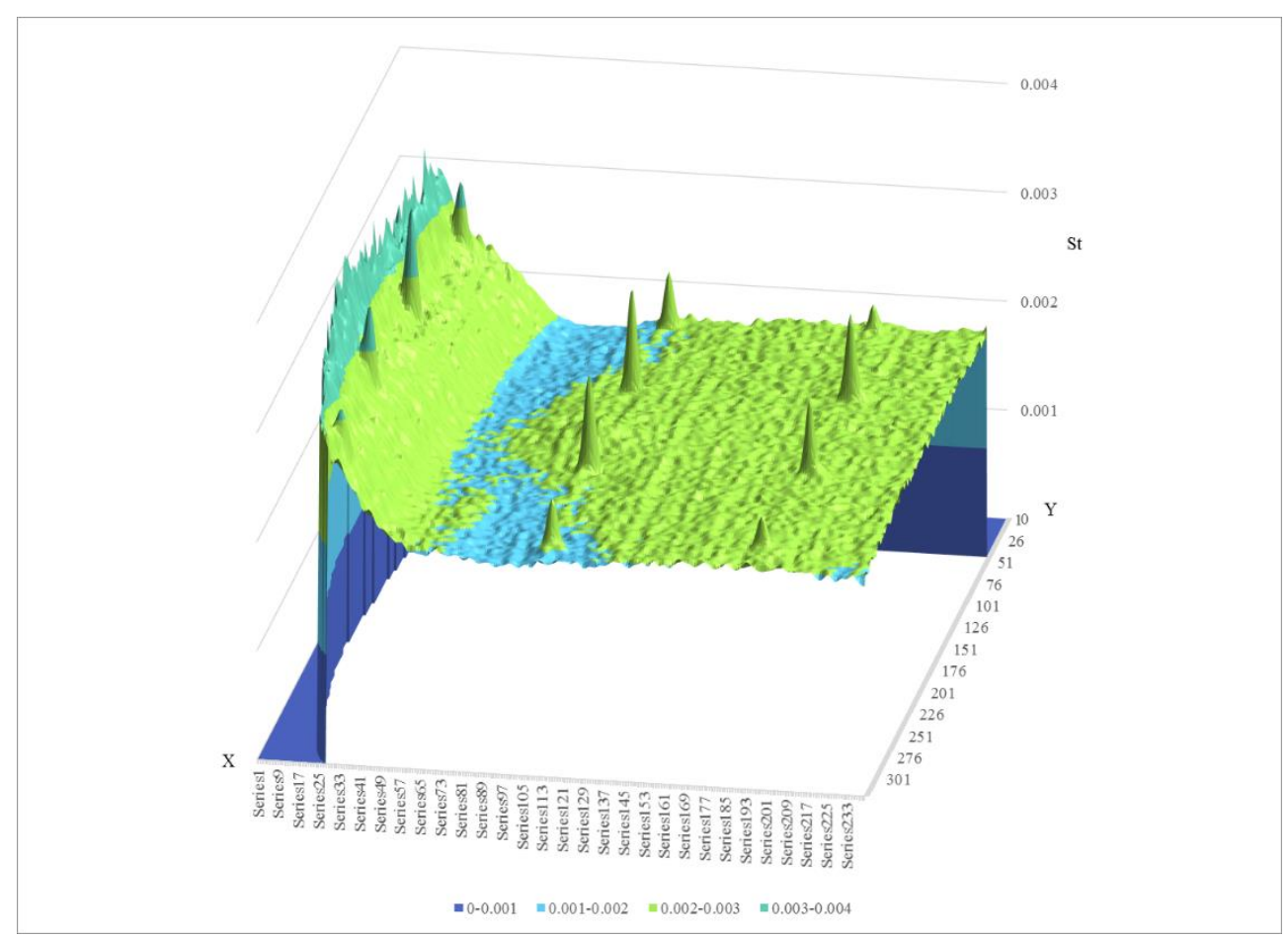

Figure 47. Suction surface Stanton number chart $\mathrm{Re}=1,000,000, \mathrm{Tu}=0.081$, unheated starting length, top 
Figure 48 show the bottom portion of the suction surface for the large grid turbulence condiditon at a Reynolds number of $1,000,000$ and a heated starting length. The flow is beginning to transition similar to that of the unheated starting length, but the differecne is there is now no rise in heat transfer at the edge of the vane. Instead the passage vortex is forcing the secondary loss core onto the surface causing a prevalent influence on the vane surface heat transfer. This passage vortex contunues to travel upward on the vane surface towards the trailing edge shifting this secondary loss core slightly towards the midspan of the vane. The peak in heat transfer near the mid span of the vane due to the increased surface roughness of the vane can still be seen. As the flow travels towards the trailing edge of the vane, figure 49, this phenomena can still be seen. Also, similar to the unheated starting length the flow has become fully turbulent.

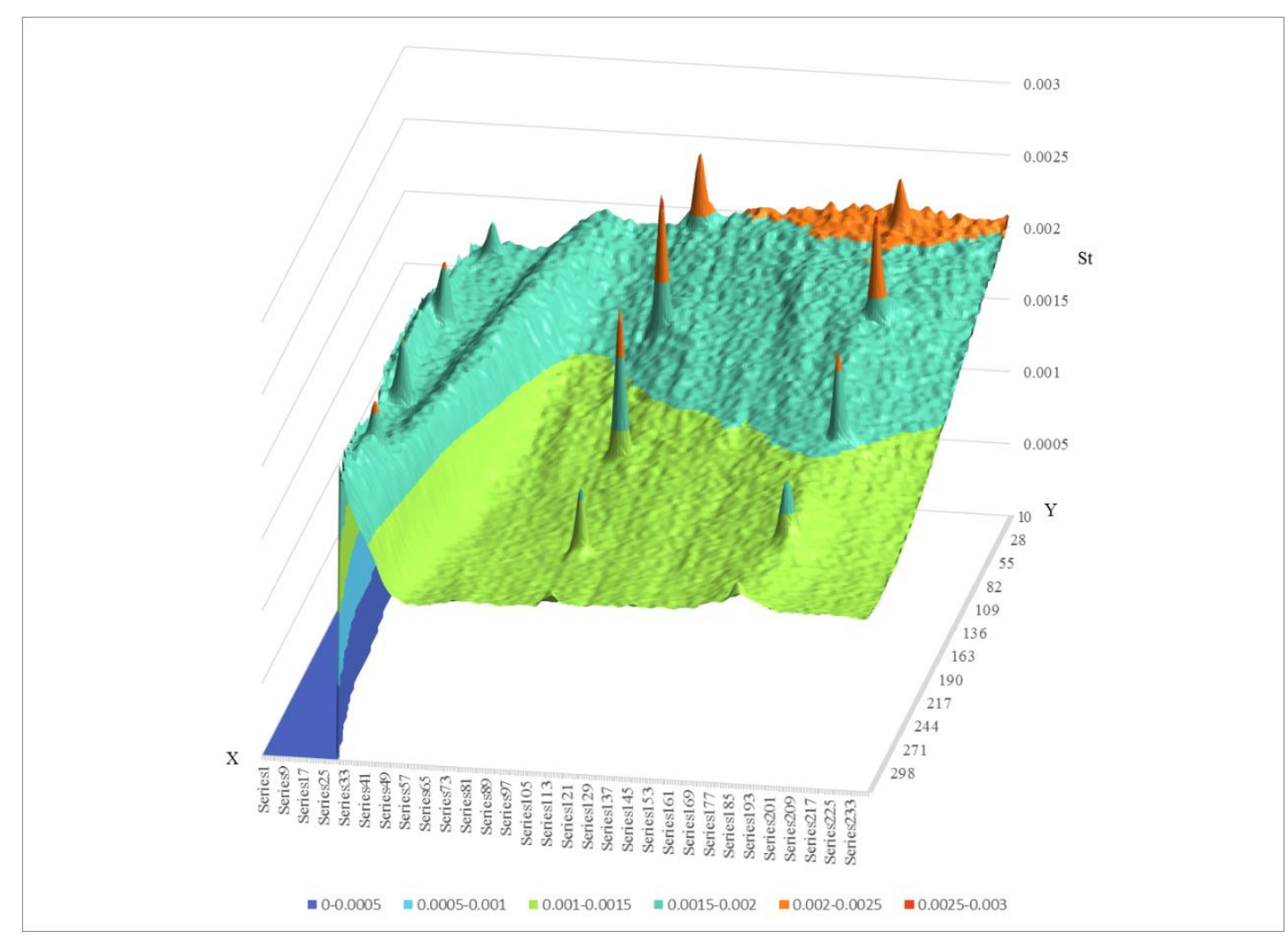

Figure 48. Suction surface Stanton number chart $\mathrm{Re}=1,000,000, \mathrm{Tu}=0.081$, heated starting length, bottom 


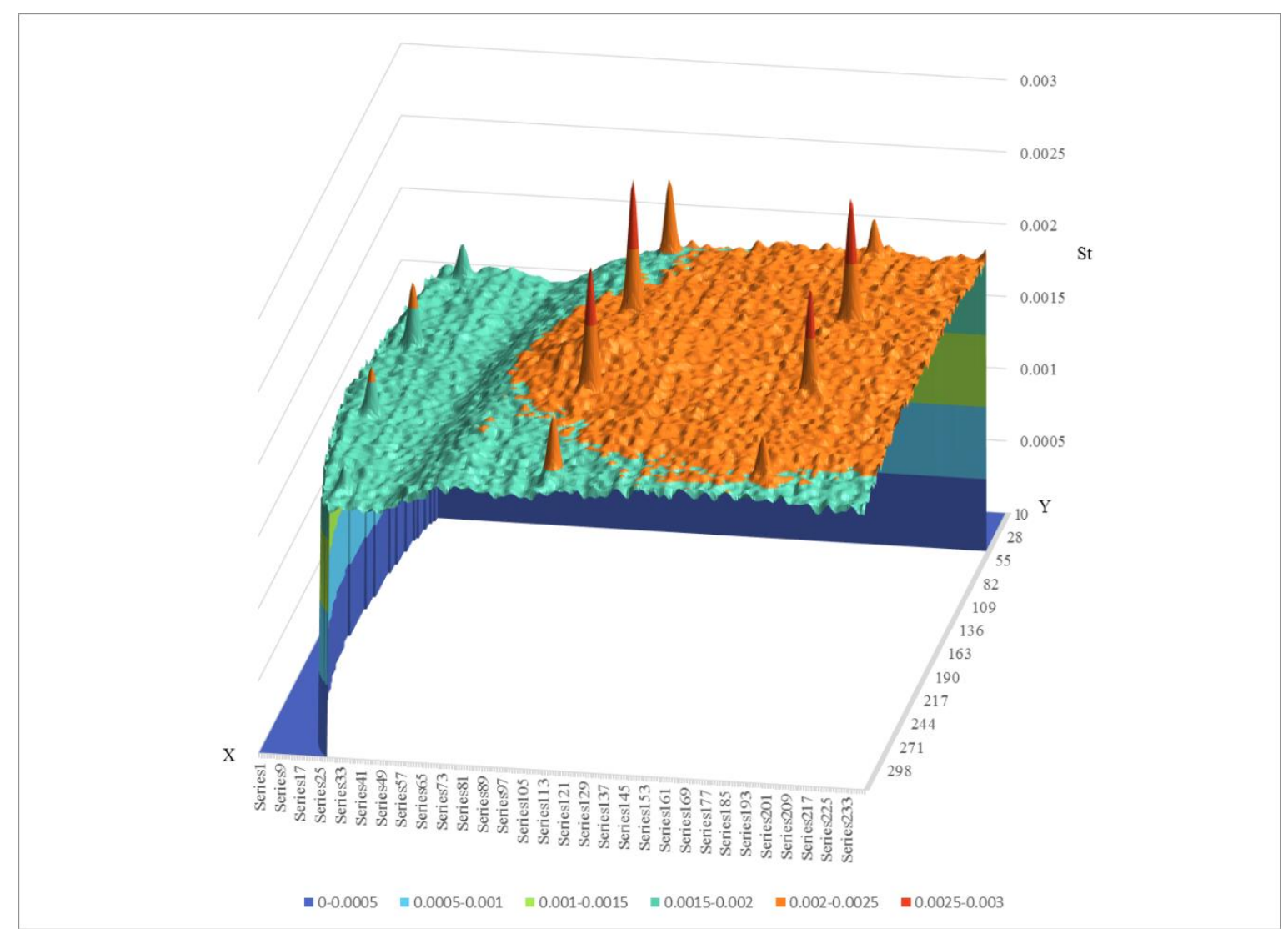

Figure 49. Suction surface Stanton number chart $\mathrm{Re}=1,000,000, \mathrm{Tu}=0.081$, heated starting length, top

When the Reynolds number decreases the same trends seen in the two-dimensional Stanton number charts can be seen in these charts. The flow for the large grid turbulence condition at a Reynolds number of 500,000 remains laminar for all of the bottom section of the suction surface, figure 50, and most of the upper section of the suction surface, figure 51. Figure 51 shows the flow beginning to transition as it reaches the trailing edge of the vane. The secondary loss core created from the passage vortex on the suction surface of the vane is still present at the lower Reynolds number, however it is difficult to see when the flow is laminar. When the flow begins to show transitional behavior, the secondary loss core becomes larger and more prevelant in the figrues. Also, there is no increase in the heat transfer levels due to surface roughness at the lower Reynolds number. 


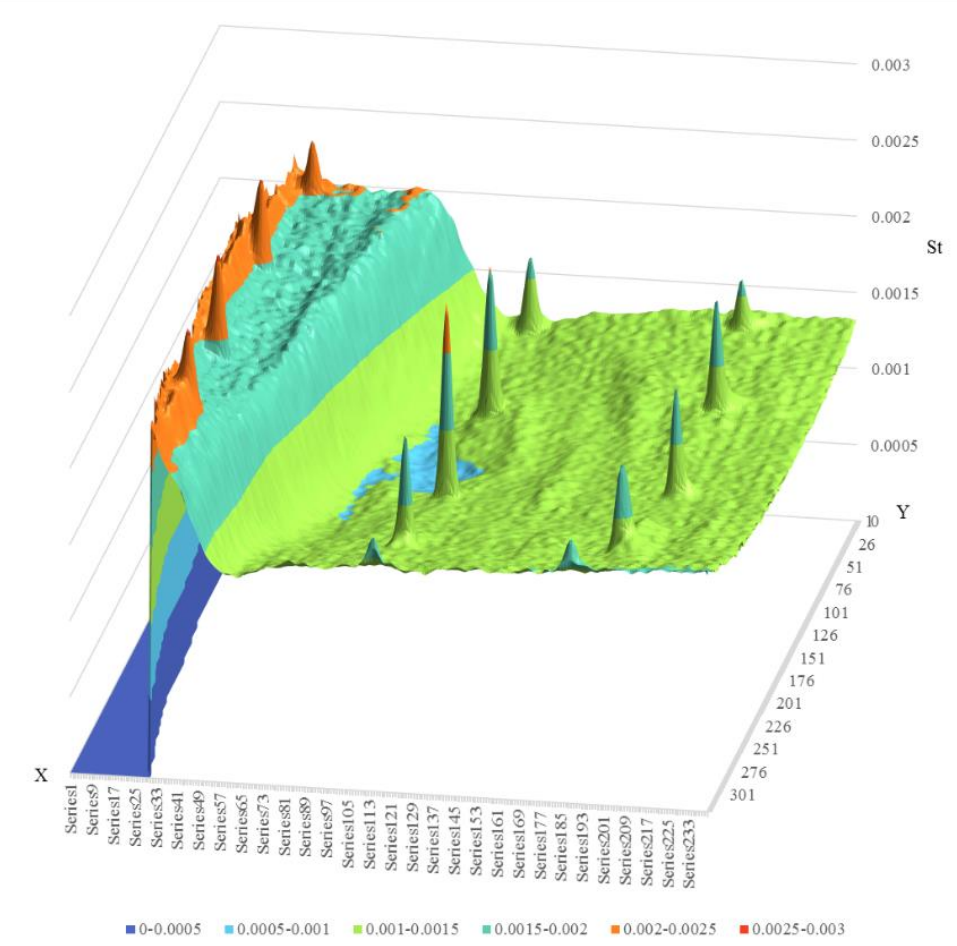

Figure 50. Suction surface Stanton number chart $\mathrm{Re}=500,000, \mathrm{Tu}=0.081$, heated starting length, bottom

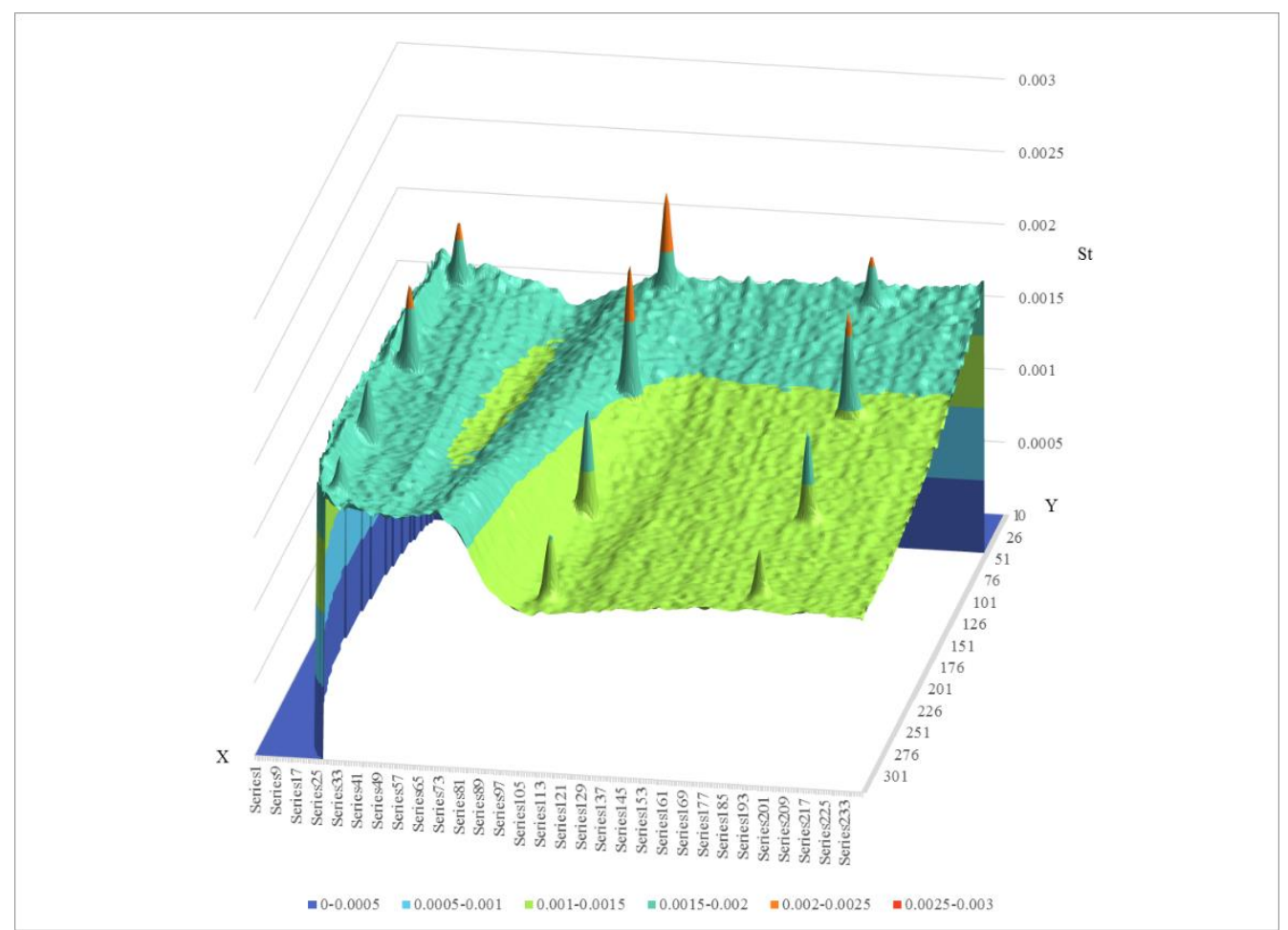

Figure 51. Suction surface Stanton number chart $\mathrm{Re}=500,000, \mathrm{Tu}=0.081$, heated starting length, top 
When the Reynolds number increases the same trends seen in the two-dimensional Stanton number charts can be seen in these three dimensional Stanton number charts. The flow for the large grid turbulence condition at a Reynolds number of 2,000,000 begins to transition earlier than when it has a Reynolds number of 1,000,000. In figure 52 the flow is in its final stages of transitional flow and becomes fully turbulent approximately half way through the figure. The passage vortex can be seen moving onto the surface of the vane and overall the heat transfer levels are faily constant. Also, the spike in heat transfer levels near the midspan of the vane due to surface roughness continues to show itself at the higher Reynolds numbers. Figure 53 shows that the flow is fully turbulent and passage vortex continues to grow on the surface of the vane. The Secondary loss core is very visible in fugure 53 due to the added growth of the passage vortex.

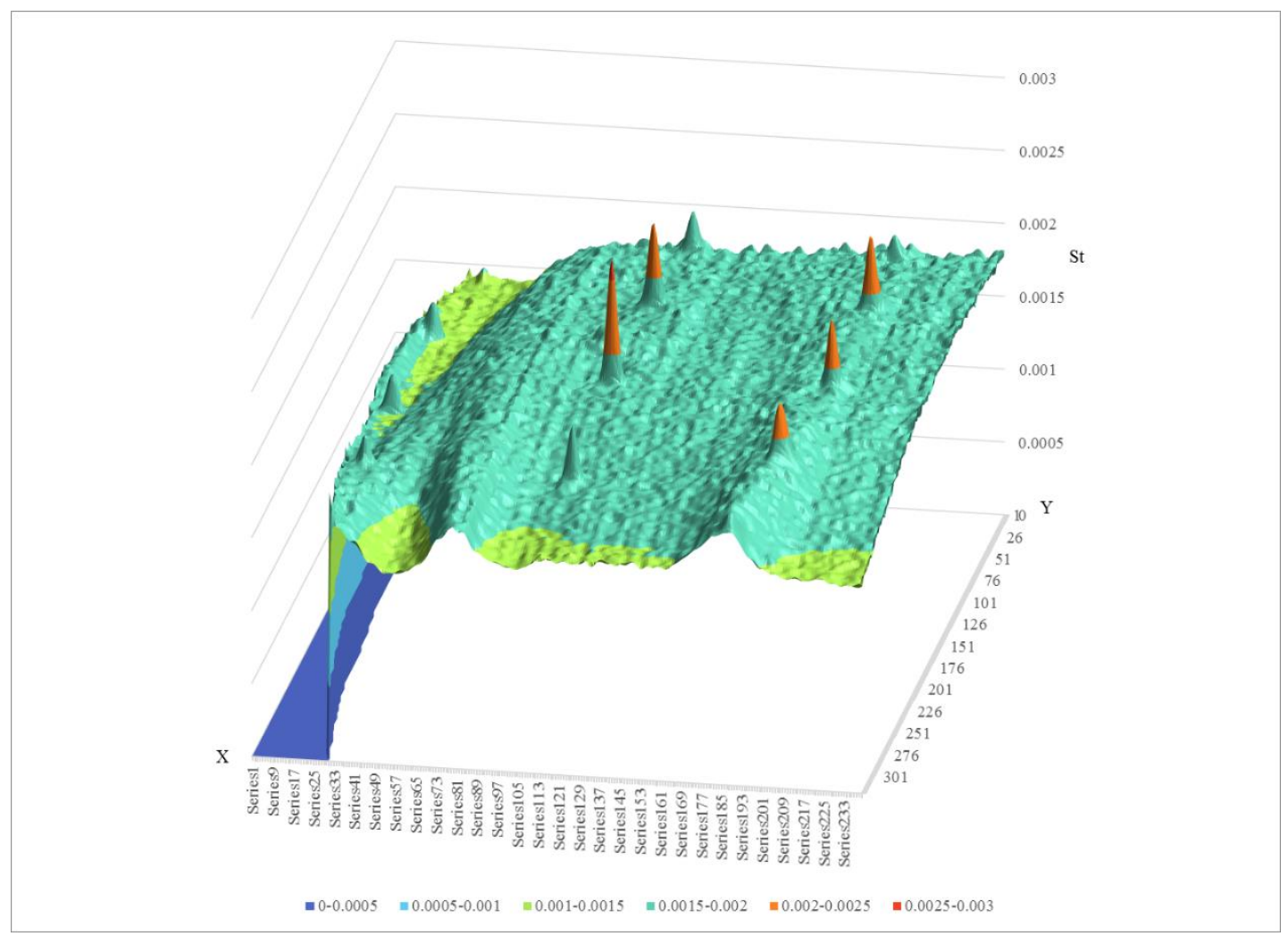

Figure 52. Suction surface Stanton number chart $\mathrm{Re}=2,000,000, \mathrm{Tu}=0.081$, heated starting length, bottom 


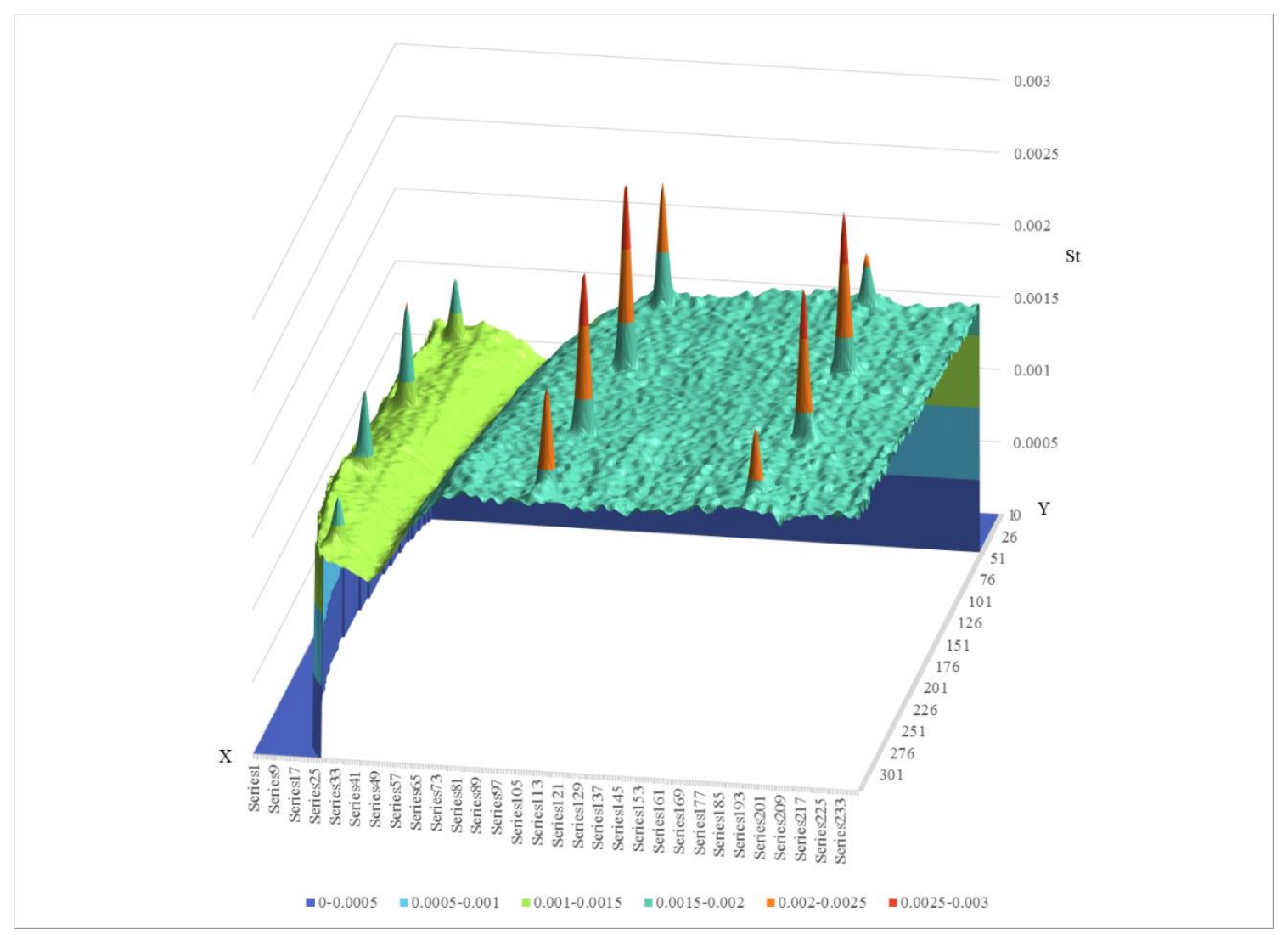

Figure 53. Suction surface Stanton number chart $\mathrm{Re}=2,000,000, \mathrm{Tu}=0.081$, heated starting length, top 


\section{CHAPTER V}

\section{CONCLUSION}

The continuing drive for more efficient and durable land based gas turbines has opened industry to explore many different designs. This experiment looked into a vane designed with a large leading edge and a suction surface that was aft-loaded. Heat transfer measurements were acquired over the surface at three different Reynolds numbers ranging from 500,000 to 2,000,000 and at seven different turbulence conditions starting at $0.7 \%$ and going as high as $17.4 \%$. The data that were collected are expected to be useful in the grounding of predictive methods for turbine airfoil heat transfer including the effects of turbulent augmentation and transition.

The combination of the large leading edge and the testing the vane at high turbulence levels provides useful data on stagnation region heat transfer and augmentation. The data that were collected were compared to predictions made based upon the TRL parameter. When comparing the current data to previous test conducted, the trends that are present in the newer data are fairly consistent with the past results. Where this current study differs is at high turbulence levels the heat transfer augmentation levels are slightly lower than what was expected. This reduction in augmentation levels is believed to be due to faster turbulence dissipation rates and longer convection times that come with a larger leading edge.

The pressure surface of the vane experiences a drop in the acceleration soon after the flow exits the stagnation region of the vane, then sees a significant increase in the acceleration levels due to the complex curvature of the vane. This differs from a conventional vane, but can help us gain a greater understanding to the trends of the heat transfer and augmentation levels 
that occur on the pressure surface of the vane. The heat transfer augmentation levels show an early a drop followed by a significant rise. A very similar trend can also be seen in the heat transfer levels. The similarity between the heat transfer and heat transfer augmentation levels can be seen. However, the augmentation levels are not fully consistent with the acceleration profile that the pressure surface experiences. Also, the pressure surface seems to have a noticeable difference between the predicted heat transfer levels and the collected data. When comparing the low turbulence condition to the predicted values an under prediction is clearly evident. This under prediction suggests the presence of Taylor Gortler vortices, or at the higher turbulence levels the effects of transitional flow could be another reason why the data are noticeably higher than the predictions.

When looking at the suction surface of the vane the Stanton number augmentation profiles show that the levels of heat transfer are below the predicted augmentation levels on the suction surface. Also, when comparing the heat transfer distributions to the predictions made the actual transition occurs well downstream from the predicted location of transition. The combination of lower augmentation levels with delayed transition show that the convex curvature of the vane has a significant impact on the levels of laminar augmentation and when transition occurs.

The three dimensional Stanton number charts show a great deal of the physics of the flow on the suction surface of the vane. The lower turbulence conditions tend to have a lower Stanton number at the mid span of the vane, similar to that of the vane heat transfer charts. However when there was an unheated starting length the heat transfer levels became higher at the unheated endwall heated vane interface. This difference can be attributed to the more coherent passage vortices at the lower turbulence levels. When the endwall heat was turned on and the vane 
experienced a heated starting length, the vane heat transfer became much more constant. The passage vortex system on the suction surface of the vane became much more visible. This passage vortex system caused the heat transfer visualization to reveal a secondary loss core which only became greater as the Reynolds number was increased. Even though the heat transfer data with a heated starting length showed secondary flow effects were present, the heated starting length showed reasonably uniform heat transfer levels across the span of the vanes after the flow had transitioned.

Overall the data for the lower turbulence levels show a strong correlation to the predictions, which provides confidence that the set up and execution of the experiment was correct. As turbulence levels increased, trends started to occur for all of the surfaces. As the Reynolds number increased the overall heat transfer levels dropped, and with the increase in turbulence the heat transfer levels were raised. Also, the flow began to transition earlier on the suction surface as the turbulence levels were increased. The heat transfer distributions that were presented along with the comparisons to the predictions that were made using a finite difference boundary layer code show some of the challenges faced when using engineering turbulence models.

\section{Future Studies}

These vane heat transfer data taken for this study will be used with a later study about vane film cooling. This vane film cooling study will also use data from an internal cooling experiment that Dr. Ames is also overseeing. The study will use a vane with the same dimensions as the vane used in this study, but focus on the effectiveness of film cooling over the surface of the vane. Additionally in the overall study the heat transfer, film cooling, and internal heat transfer data will tie together to form the design used for cooling this vane. The 
configuration of the internal cooling parts of the vane will be determined by data taken Abdul Sheikhmohamed and analyzed by Loren Soma. By combining the heat loads taken from the cascade data and the cooling data from the other study this future study should be able to provide a vane cooling effectiveness level with little variance over the entire surface of the vane. 
APPENDICIES 


\section{APPENDIX A}

\section{CASCADE DIMENSIONS}

All of the units for the cascade dimensions are presented in inches.

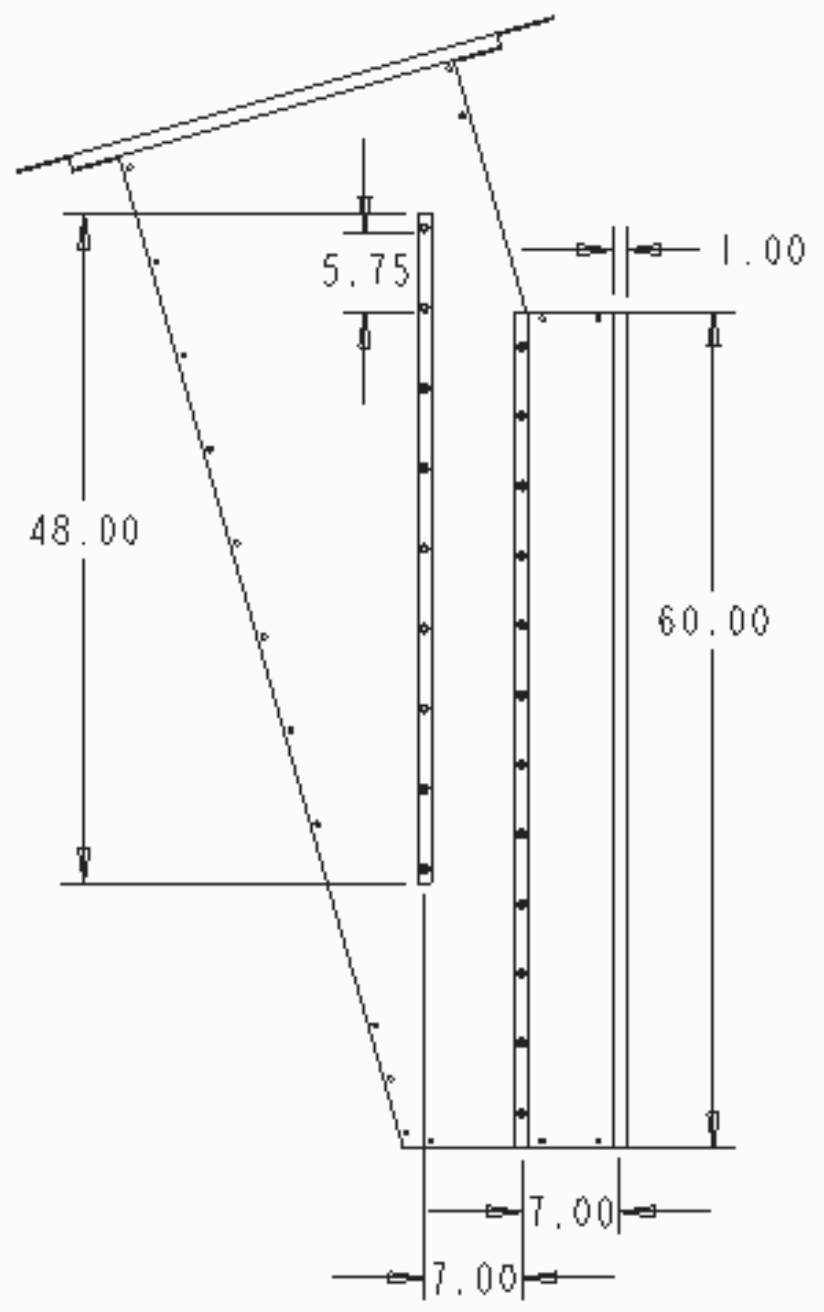

Figure 54. Cascade far endwall dimensions 


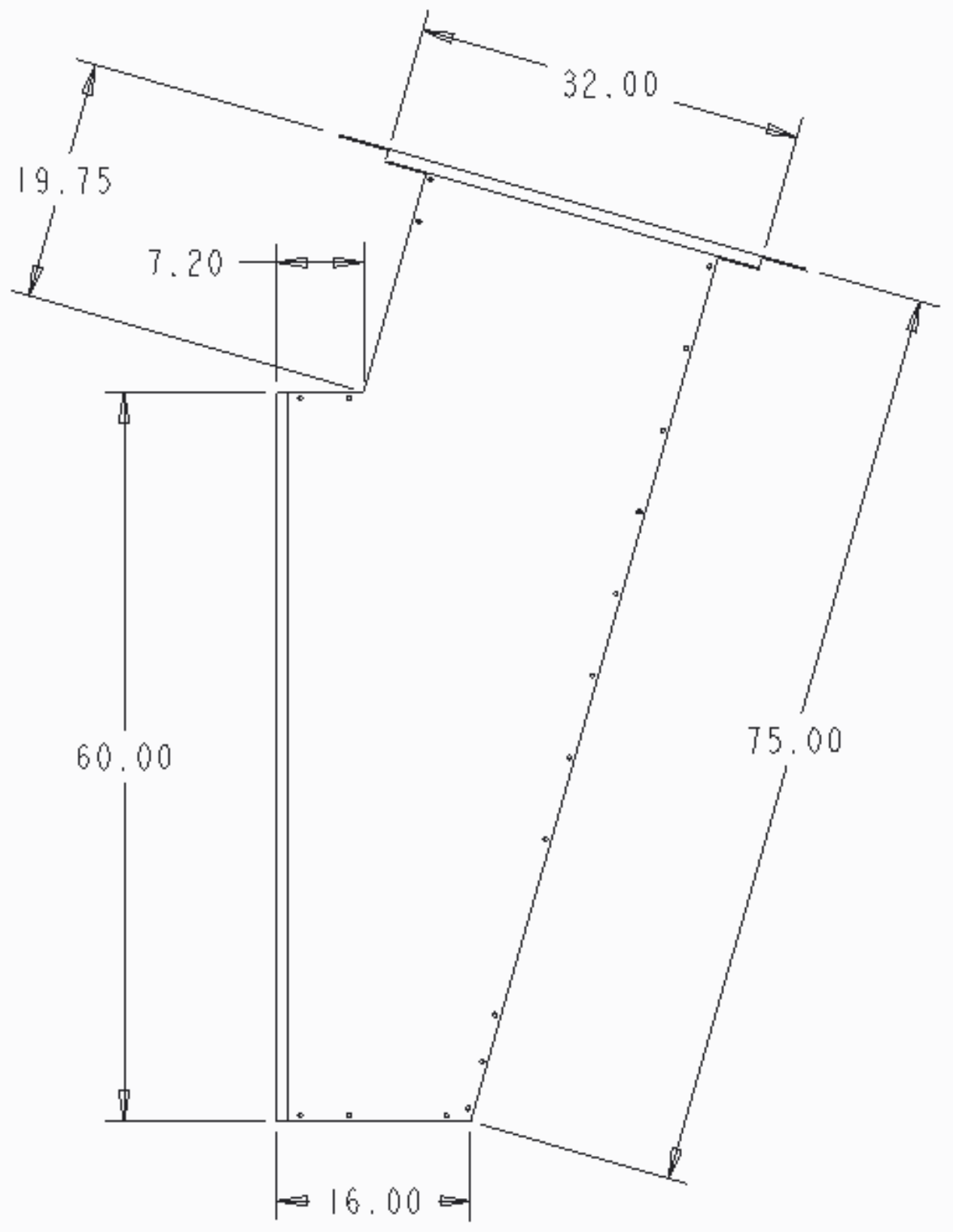

Figure 55. Cascade near endwall dimensions 


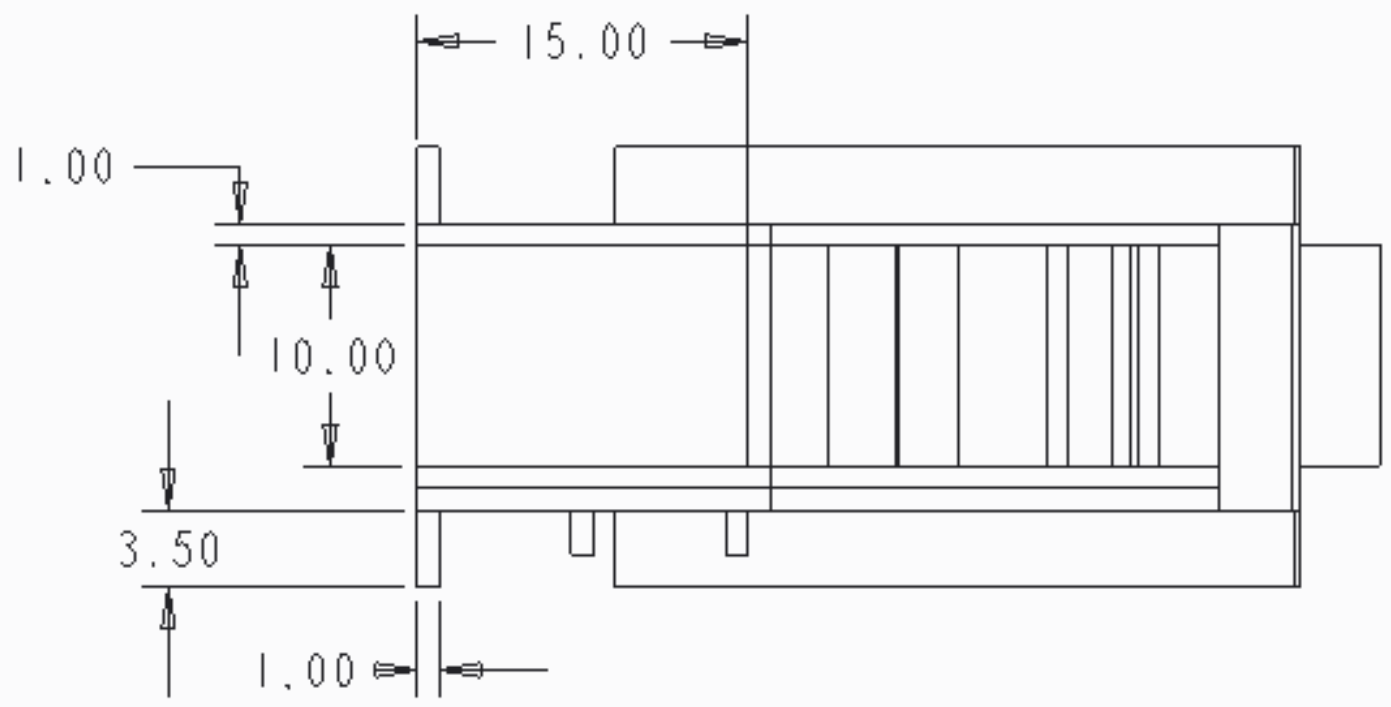

Figure 56. Cascade bottom dimensions

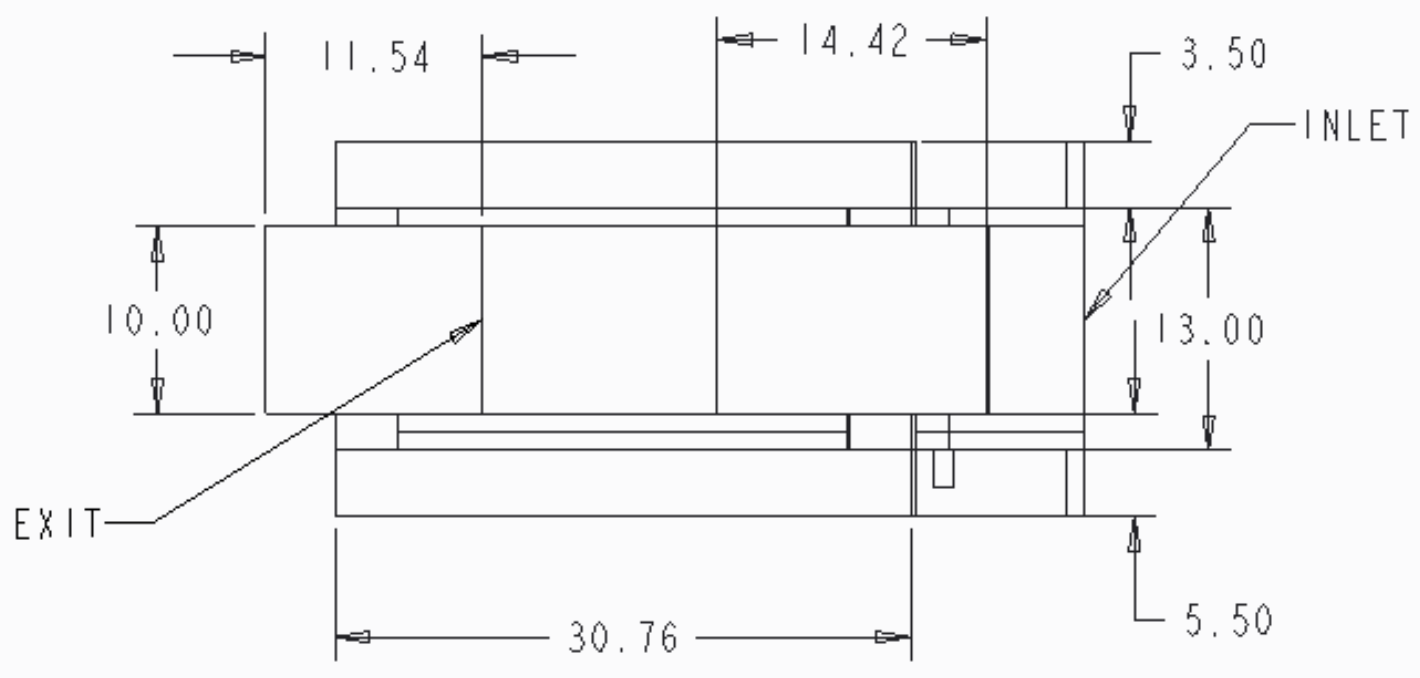

Figure 57. Cascade top dimensions 


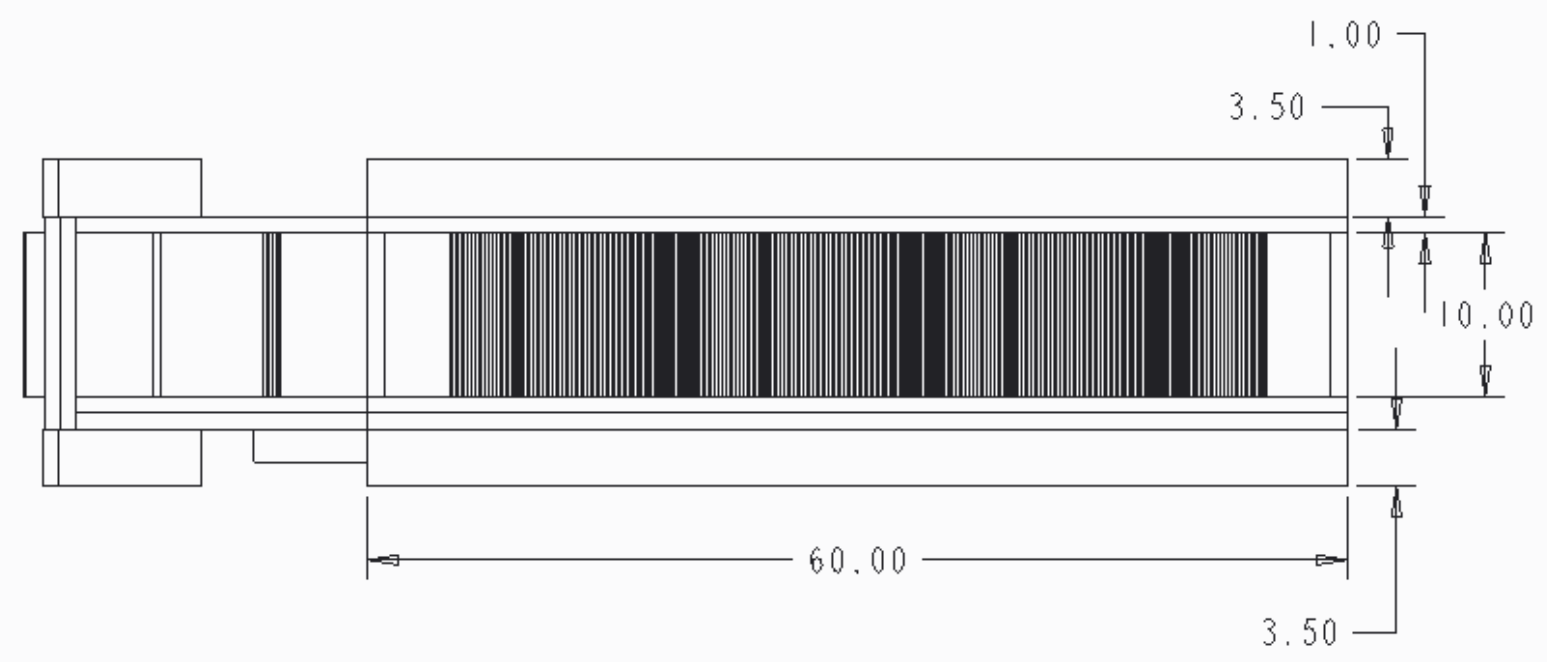

Figure 58. Cascade front dimensions

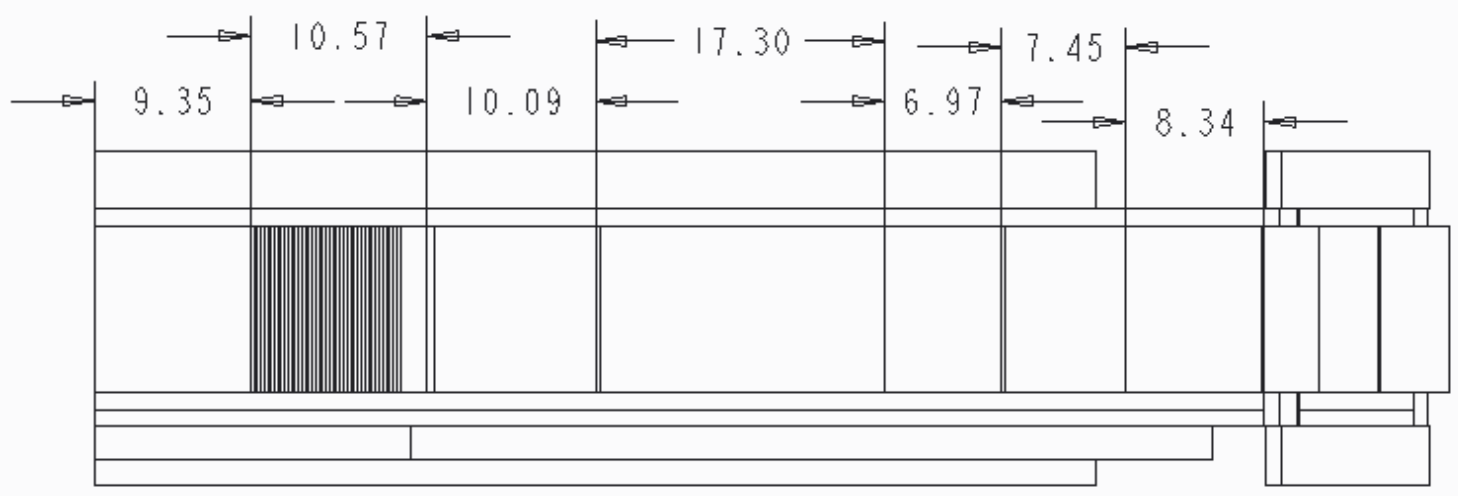

Figure 59. Cascade back dimensions 


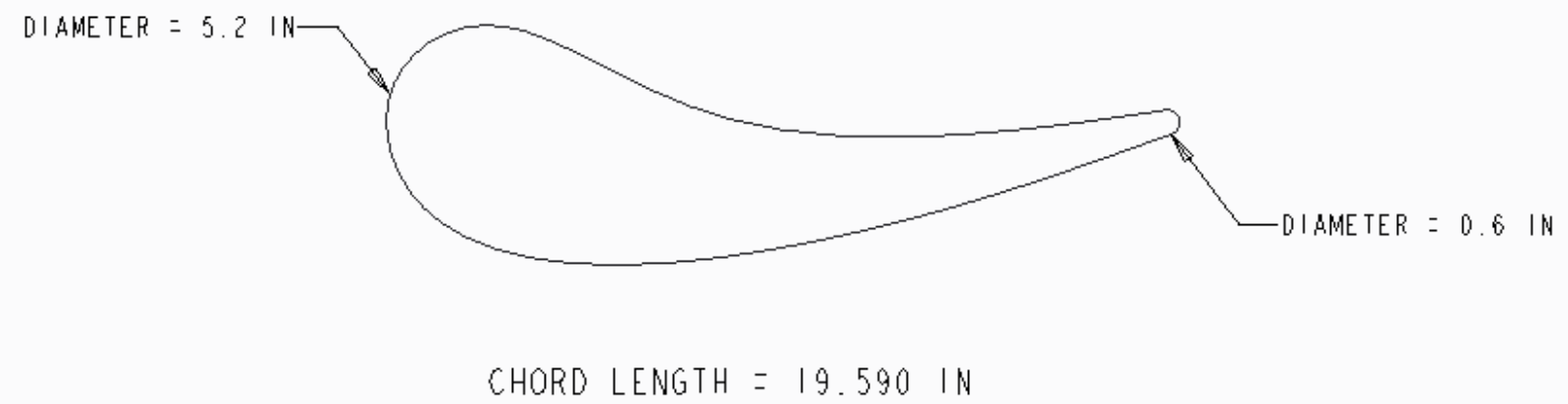

Figure 60. Vane dimensions 


\section{APPENDIX B}

\section{FABRICATION OF VANES}

This Appendix gives a detailed description of the process that was used to fabricate the vanes used in the cascade. Before the vanes were cast a foam core had to be constructed. The core, which was 1/16 of an inch smaller in radius from that of the finished vane, was routered from 1 inch thick isocyanurate foam. After ten pieces of foam were routered, they were then glued together and cut to the desired height of 9.5 inches. Spacers, made from acrylic and cut to a height of $1 / 4$ of an inch, were then glued to the top and the bottom of the foam core to allow the epoxy flow under the core during the casting process. This ensured the strength of the vane would be adequate when the casting process was completed. The next step, before the vane could be cast was to sand down the trailing edge. Again this was so more epoxy could flow in that area in the mold, increasing the strength in the trailing edge. Another change that was done to the foam core before it could be cast was to drill two 1.5 inch diameter holes out of the first 1.5 inches of the top of the foam core. This allowed a place for two threaded holes to be placed on the vane. These threaded holes were then used to bolt the vane onto the endwall of the cascade. The last thing that was done to the core before it was cast was, a thru hole was drilled through the middle of the core. This allowed a place where a funnel could be inserted and the epoxy could be poured. This ensured that the epoxy reached the bottom of the mold during the casting process.

When the fabrication of the foam core was completed and the casting process was about to begin the core was placed in a mold. The mold consisted of 12 separate plates on both the pressure surface of the vane as well as the suction surface. The top plate of the mold, on both the pressure surface and suction surface, had radius that was $3 / 4$ of an inch larger the rest of the 
plates. This created a flange on the vanes, which was an important part in securing the instrumented vanes to the cascade. The top plate was only used in the casting of the instrumented vanes. On the mold for vanes there were two alignment holes on each of the plates which helped aid in the alignment of the mold. Along with the two alignment holes there were three holes where $1 / 2$ inch threaded rods could be inserted and condense the mold. This helped stop any epoxy from seeping into the cracks between the aluminum plates. Each of the plates was fabricated from 1 inch thick 6061 Aluminum on a CNC machine. A bottom 1 inch thick plate was also fabricated to provide a base for the mold and a help aid in the alignment of the plates.

When the fabrication of the foam core was completed and the mold was constructed, the epoxy could be poured. The epoxy used in the casting process was a two part epoxy consisting of a resign and a hardener. The characteristics that were desired for the epoxy was a low viscosity, low exotherm, transparent, and suitable strength, the combination of thermoset 600 resign and number 64 hardener gave us these properties. When this specific resign and hardener were combined using the correct proportions, 74 percent hardener and 26 percent resign, they had a working life of 3 hours and a cure time of 48 hours. The reason an epoxy with a long working time was chosen was that a low viscosity epoxy needs a long working life to ensure a high surface quality. After the epoxy was thoroughly mixed together it was placed in a vacuum to allow any air bubbles created during the mixing process to be eliminated from the fluid. This was important because the presence of air bubbles in the epoxy could cause a poor surface finish for the vane. The low viscosity properties of the liquid epoxy allow the air to easily rise to the surface when placed in the vacuum, so the removal is not overly difficult. By placing the liquid epoxy in a vacuum of up to 26 inches $\mathrm{Hg}$ for 20 to 30 minutes the air bubbles could be 
completely removed. Since our epoxy had a working life of 3 hours, the time in the vacuum was not an issue.

Once the epoxy was mixed and taken out of the vacuum, the pouring process could begin its final step. The mold had to be leveled and the foam core had to be anchored down inside the mold. This was because the foam core would float in the epoxy if not forced down to the bottom of the mold. Two wooden rods were wedged between a counter, which was above the mold, and the foam core. This ensured that the foam core was at the bottom of the mold throughout the entire casting process. After the vane was cast the wooden rods were cut off of the vanes. When the vane was secure the epoxy could start to be poured. A funnel was placed in the thru hole drilled on the top surface of the foam core and the epoxy was slowly poured into the mold. At this point it was very important to maintain a constant pouring velocity so no air bubbles were created, to ensure a good surface finish. However, if any air bubbles were created the low viscosity properties of the liquid epoxy allowed the trapped air to rise to the surface before it hardened. After all of the epoxy was poured into the mold the vane was allowed to cure for 48 hours to ensure a finished product.

When the epoxy finished curing the vane could then be taken out of the mold. This process was quite delicate because the epoxy had some tendency to want to stick to the mold. To overcome this, a mold release was painted onto all surfaces of the mold, but the process still had to be done carefully to ensure no harm was done to the vane. To open the mold, one would take a wedge that screwed open was used to slowly pry the mold apart. This process was delicate at the beginning, but when a larger gap opened between the plates of mold the vane came out easily. When the vane was completely out of the mold it had to be machined down to a precise 10.0 inches, and the surface of the vane cleaned of any fins that were created from the epoxy trying to 
seep through the cracks between the plates in the mold. The fins were taken off by taking a razor blade along the surface and gently cutting away the excess epoxy, making sure not to alter the shape of the vane in any way. Once the fins were off of the vane and it was machined down to a precise 10.0 inches the vane was ready to be place in the cascade and bolted into place. 
APPENDIX C

\section{THE ENDWALL HEAT TRANSFER SYSTEM}

This appendix gives a more detailed description of the endwall heat transfer system used in this study. The system used consists of the three vane shaped heat sinks placed into the far endwall of the cascade, and a blower which supplies air to these heat sinks. The endwall heat sinks were placed in the far endwall of the cascade. There main objective was to help cool the surface under the vanes that were placed on top of the two Inconel foils placed on the far endwall. To do this the temperature inside the heat sinks, which were monitored by two type $\mathrm{k}$ thermocouples, were to be very similar to those of the surrounding thermocouples that were being monitored on the far endwall of the cascade. If there were any differences between the thermocouples of the endwall and those of the heat sinks the amount of air being forced through the heat sinks could be adjusted altering the temperatures of the heat sinks to their desired value.

The cooling air that was used in the cooling process was provided from second blower. This second blower was also a New York Company Bower, but it had a $3 \mathrm{hp} \mathrm{motor} \mathrm{that} \mathrm{operated}$ at $3600 \mathrm{rpm}$. From the blower the air traveled through a flexible four inch tube which connected to a 4 inch diameter polyvinyl chloride (PVC) pipe that stood upright. The height of the tube was approximately 6 feet tall, which was high enough to bring the air to the highest endwall cooler on the cascade. The vertical standing PVC pipe had three exit ports that were made from $3 / 4$ inch brass tubing and were glued in place. It also had one smaller exit port made from $1 / 2$ inch brass tubing. This $1 / 2$ inch exit port had a flexible rubber hose attached to it which extended to the back of the cascade. There it attached to a different $1 / 2$ inch brass tube which released the air from the blower onto a small surface of the endwall foil which traveled under the tailboard. Larger flexible rubber hosing was attached to the $3 / 4$ inch brass tubing on both the inlet of the endwall 
cooler and the exit ports on the vertical PVC pipe to finish the process of bringing the air from the blower to the endwall coolers.

The flow from the blower was monitored through a variable frequency, however this variable frequency drive was different than the one that monitored the flow going into the cascade. This helped us alter the airflow through the coolers and maintain a constant temperature for the endwall. If the flow that traveled through the coolers was not cooling the surface efficiently enough there was a small heat exchanger that could be turned on, which helped aid in cooling the surface to the desired temperature.

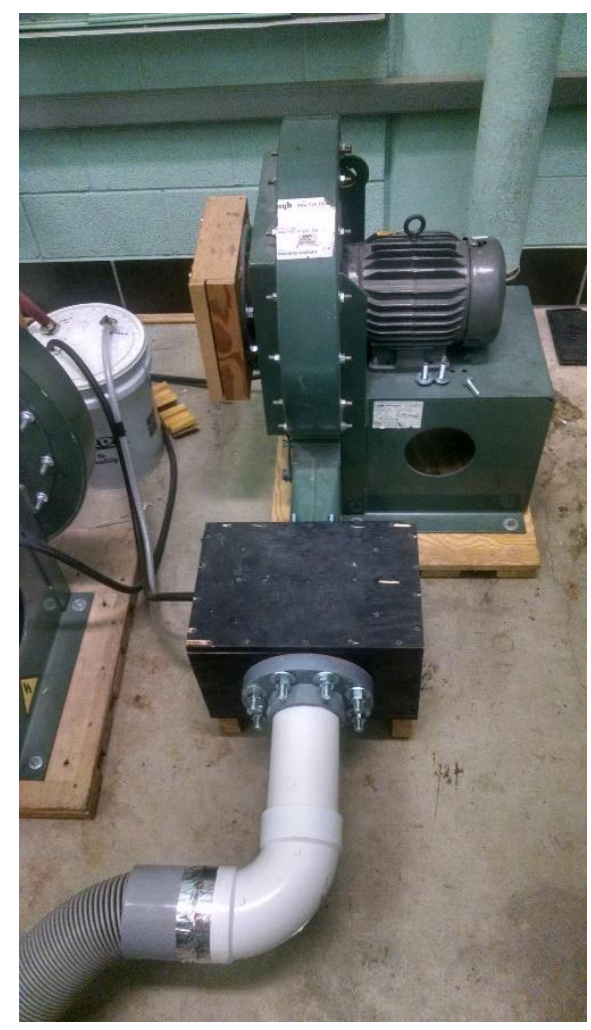

Figure 61. Endwall blower and heat exchanger

The endwall heat sinks, or endwall coolers, that the air was supplied to were manufactured at the University of North Dakota. They consisted of two different pieces the bottom, which was the main piece, and a cover. Both were made from aluminum due to its high thermal conductivity. Combined they were 0.9375 inches in height, this way they sat within the 
endwall without altering the surface above it in any way. The bottom piece was 0.75 inches in height. It was shelled out so the inside had a height of 0.625 inches. To disperse the air inside the cooler, the shelled area had 0.5 inch diameter pedestals, which were 0.5625 inches in height, and spaced 0.9375 inches in both the $\mathrm{x}$ and $\mathrm{y}$ directions. This spacing started just after the leading edge and ended just before the trailing edge leaving a gap for the inlet and exit of the air through the cooler. It was also designed for the pedestals not to constrict the flow by over 50 percent, meaning that the area for the airflow always had to be greater that the area constricted by the placement of the pedestals.

The top piece which sat 0.1875 inches in height, had a small 0.0625 inch lip manufactured into it so it would sit on top of the pedestals, but also remain flush with the top of the bottom piece. This helped with the alignment of the cover on the cooler. The cap also had an inlet and exit port manufactured into it. The port was 0.25 inch high and 1 inch in diameter cylinder that was placed in the hollow areas that were created from the void of pedestals in the leading edge and trailing edge of the bottom piece. Then a 0.714 inch thru hole was drilled out of the center of the cylinder creating the inlet and exit ports. To help with securing the brass fittings that were placed in the inlets and exits, $1 / 2$ inch pipe threads were threaded into the remaining aluminum that was left of the cylinder. The inlet port was then connected to one of the three rubber tubes coming out from the vertical PVC pipe, and the exit port was open to the atmosphere for the air to escape. This system allowed the endwall under the vanes to be properly cooled to the surrounding endwall temperature ensuring that the heated endwall data that was collected was accurate. 


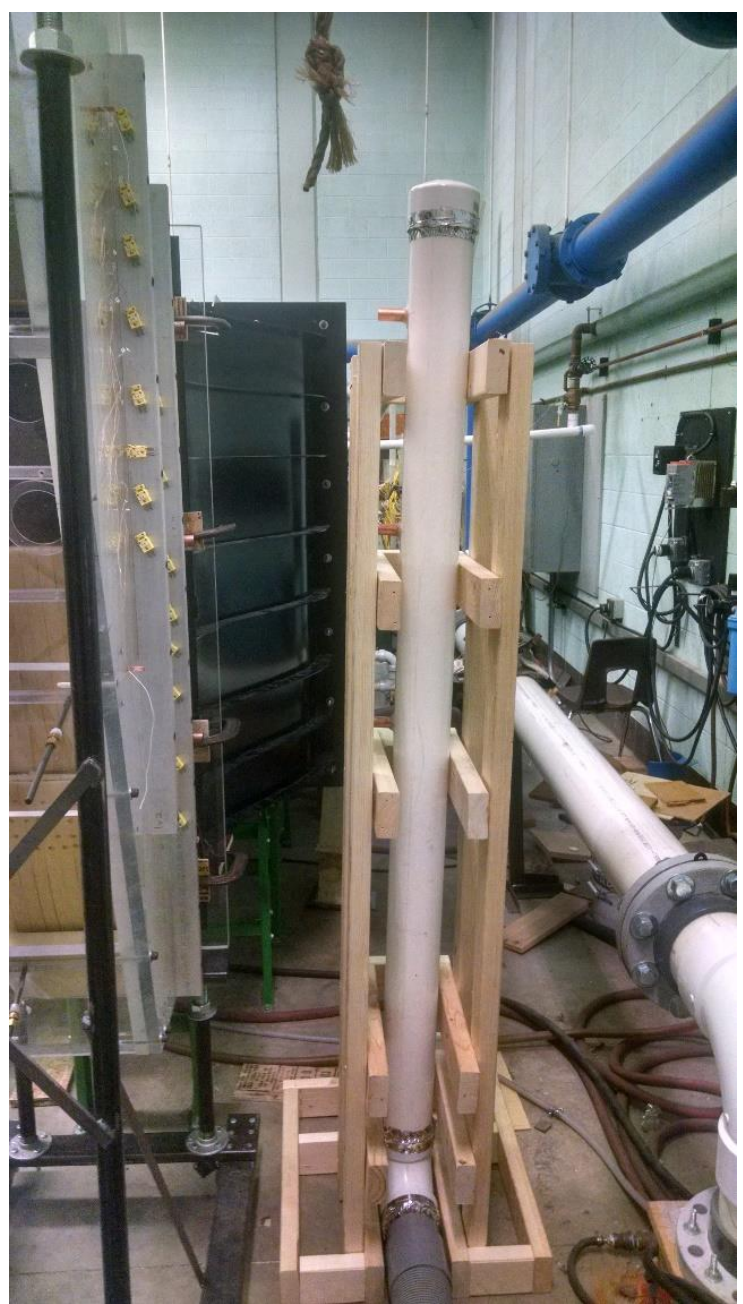

Figure 62. Endwall cooling air supply 


\section{APPENDIX D}

\section{SUCTION SURFACE STANTON NUMBER CHARTS}

For all of the figures in this appendix the $\mathrm{x}$-axis on the figures represents the spanwise direction on the vane where the pixel value of 1 represents the edge of the vane and 240 represents the area near the midspan of the vane. The y-axis on the figures represents the streamwise direction of the flow with the flow entering the figure at the pixel number of 320 and exiting the figure at 1.

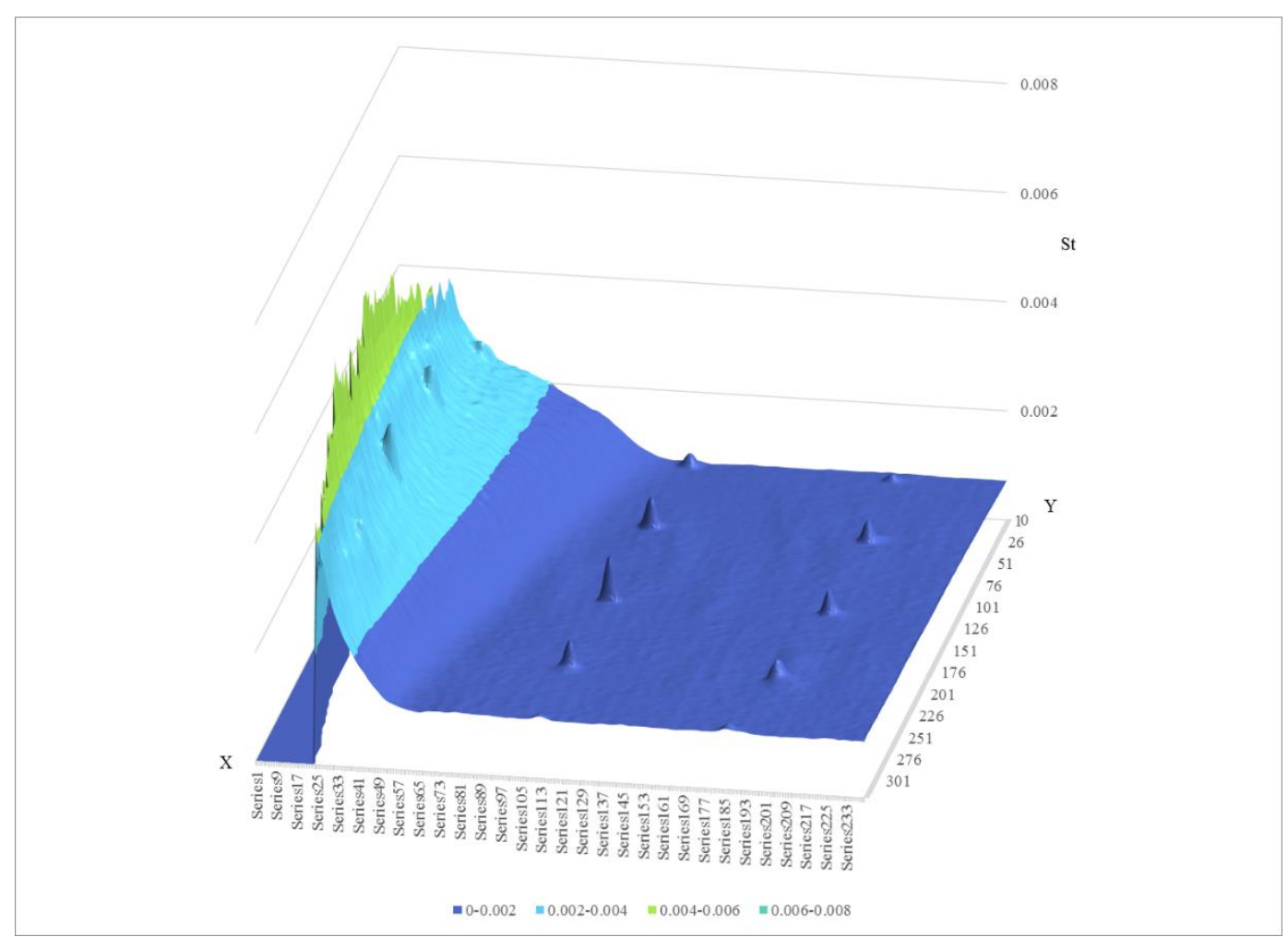

Figure 63. Suction surface Stanton number chart $\mathrm{Re}=500,000, \mathrm{Tu}=0.035$, unheated starting length, bottom 


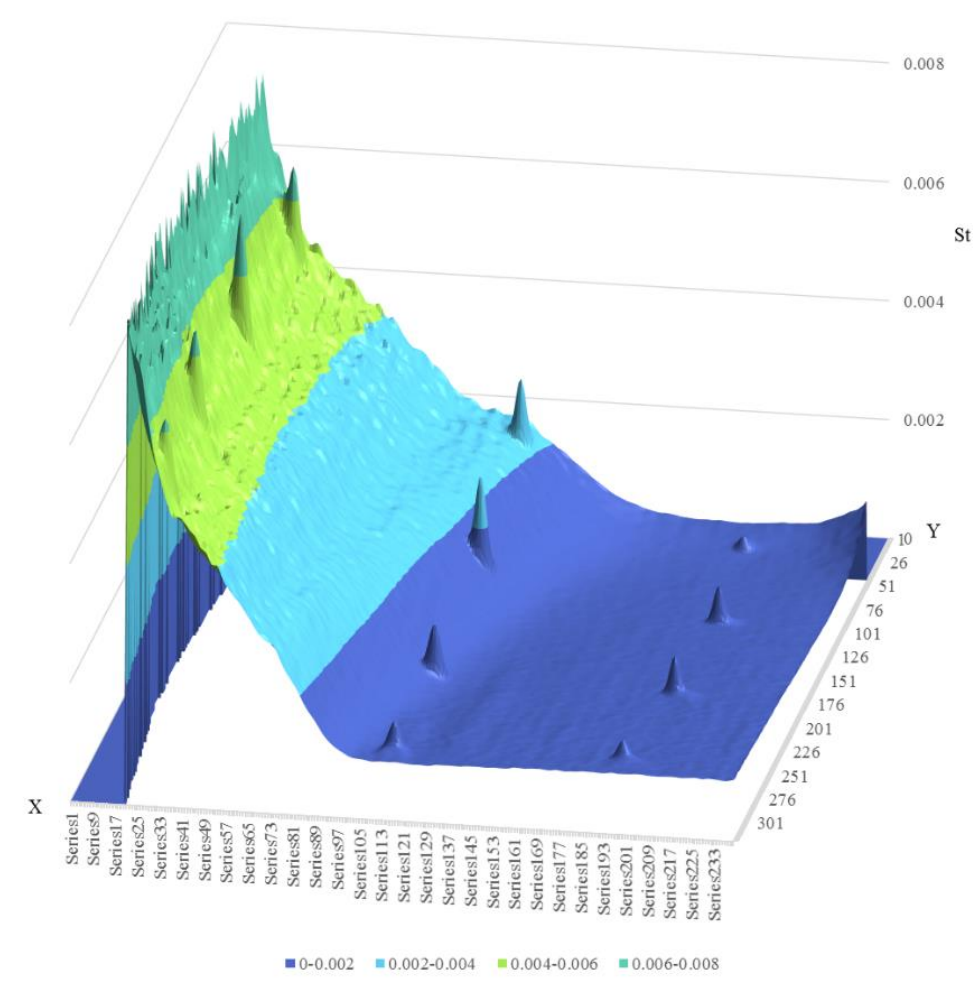

Figure 64. Suction surface Stanton number chart $\mathrm{Re}=500,000, \mathrm{Tu}=0.035$, unheated starting length, top 


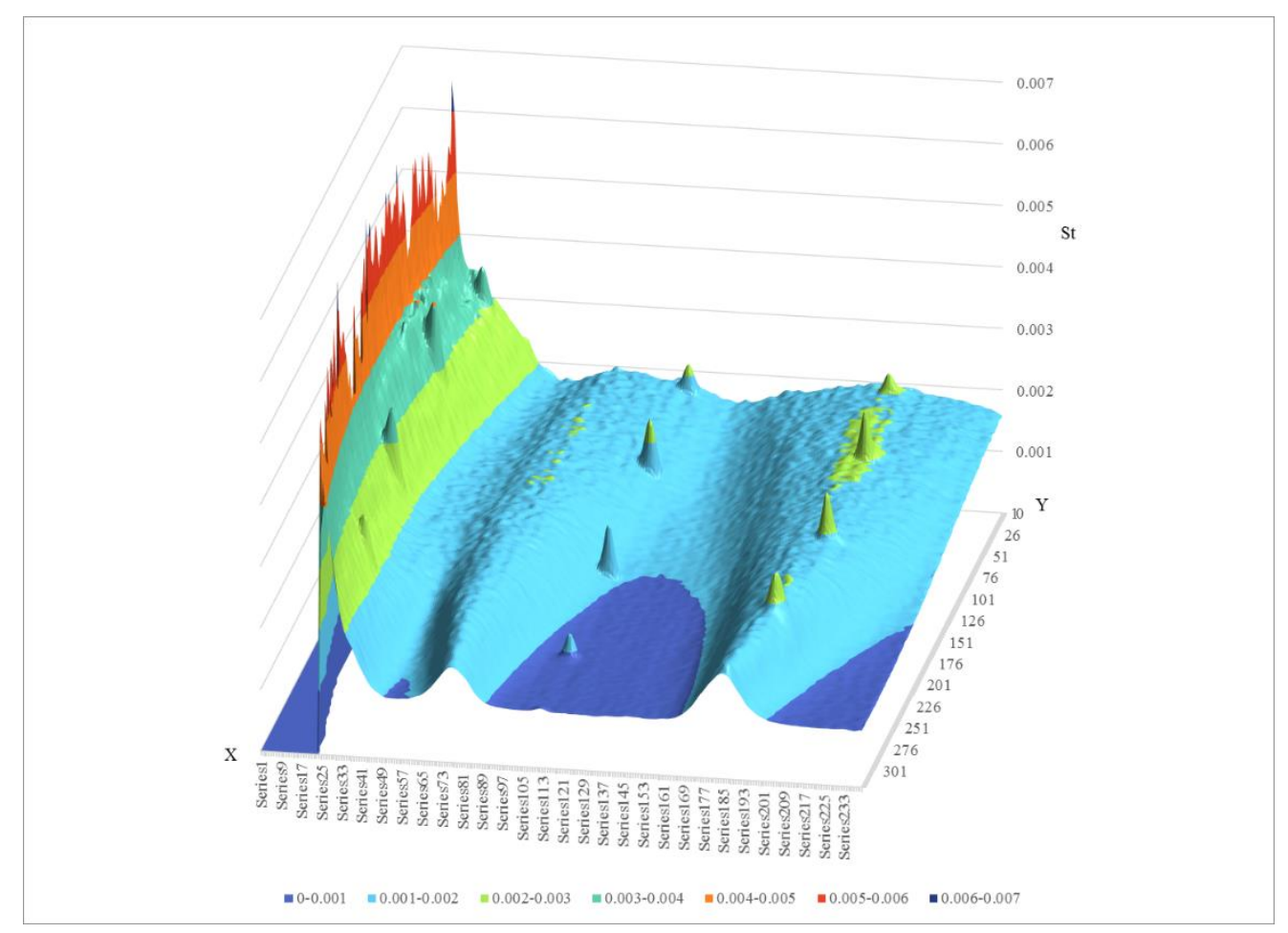

Figure 65. Suction surface Stanton number chart $\mathrm{Re}=2,000,000, \mathrm{Tu}=0.035$, unheated starting length, bottom

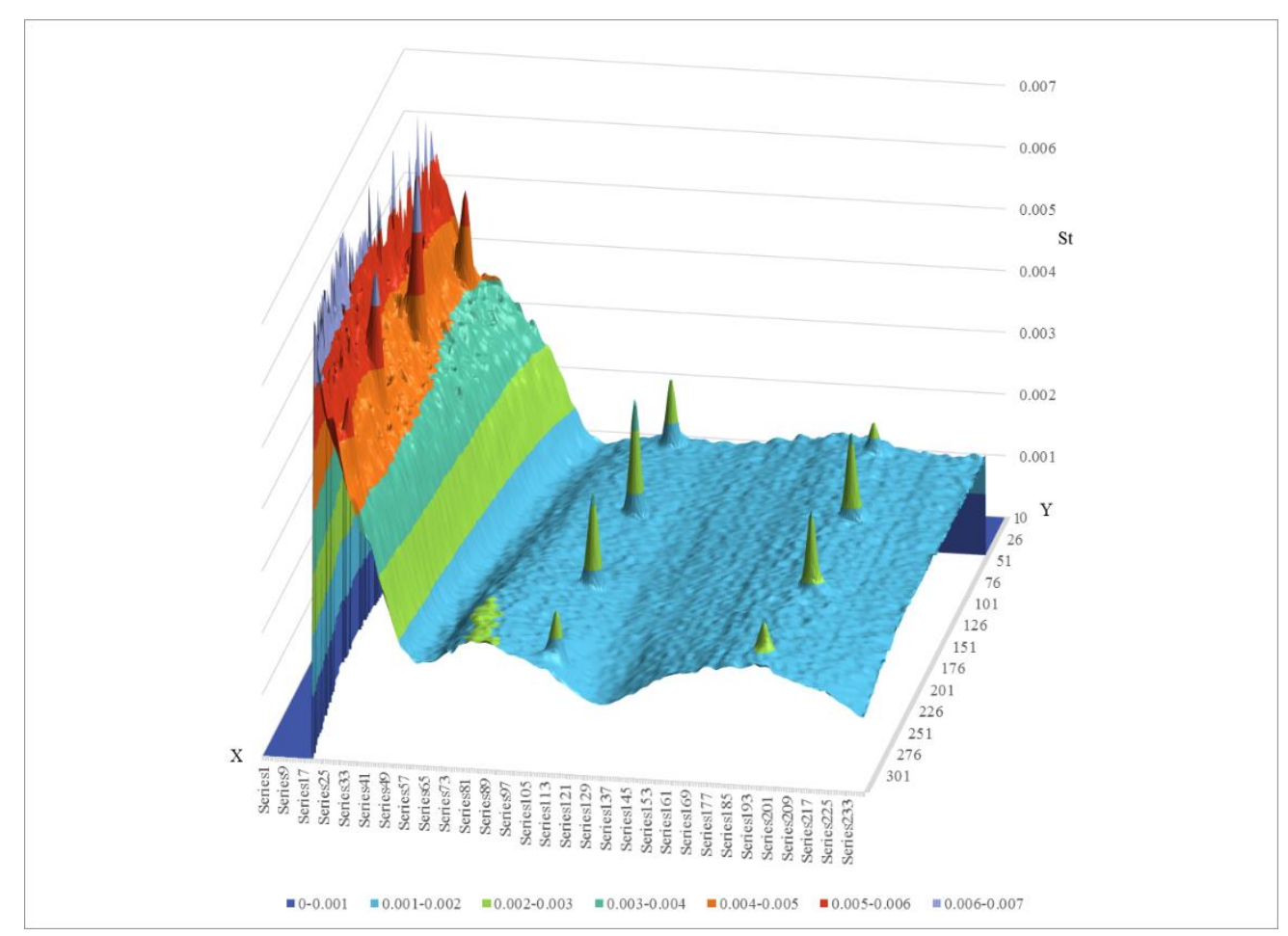

Figure 66. Suction surface Stanton number chart $\mathrm{Re}=2,000,000, \mathrm{Tu}=0.035$, unheated starting length, top 


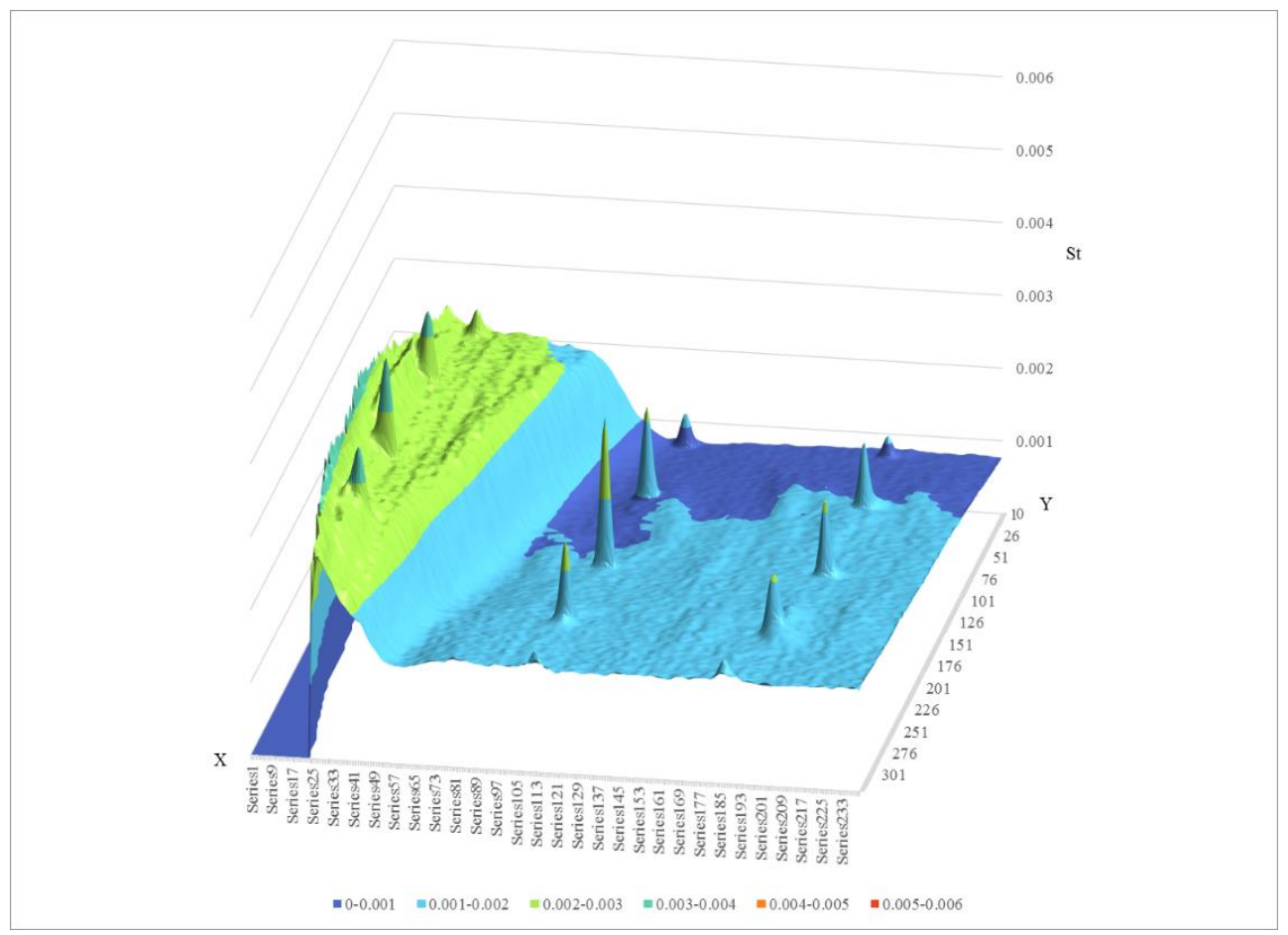

Figure 67. Suction surface Stanton number chart $\mathrm{Re}=500,000, \mathrm{Tu}=0.035$, heated starting length, bottom

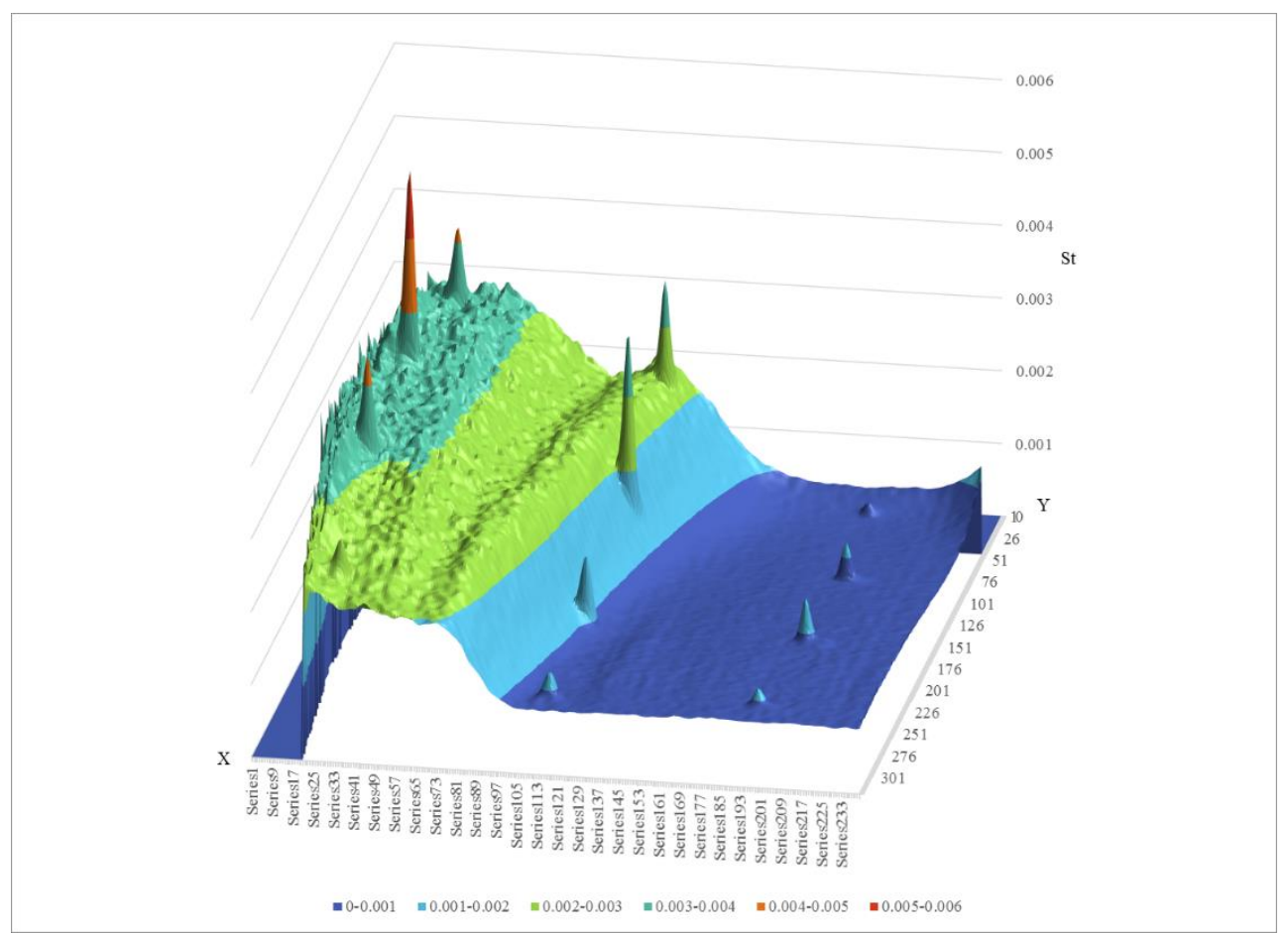

Figure 68. Suction surface Stanton number chart $\mathrm{Re}=500,000, \mathrm{Tu}=0.035$, heated starting length, top 


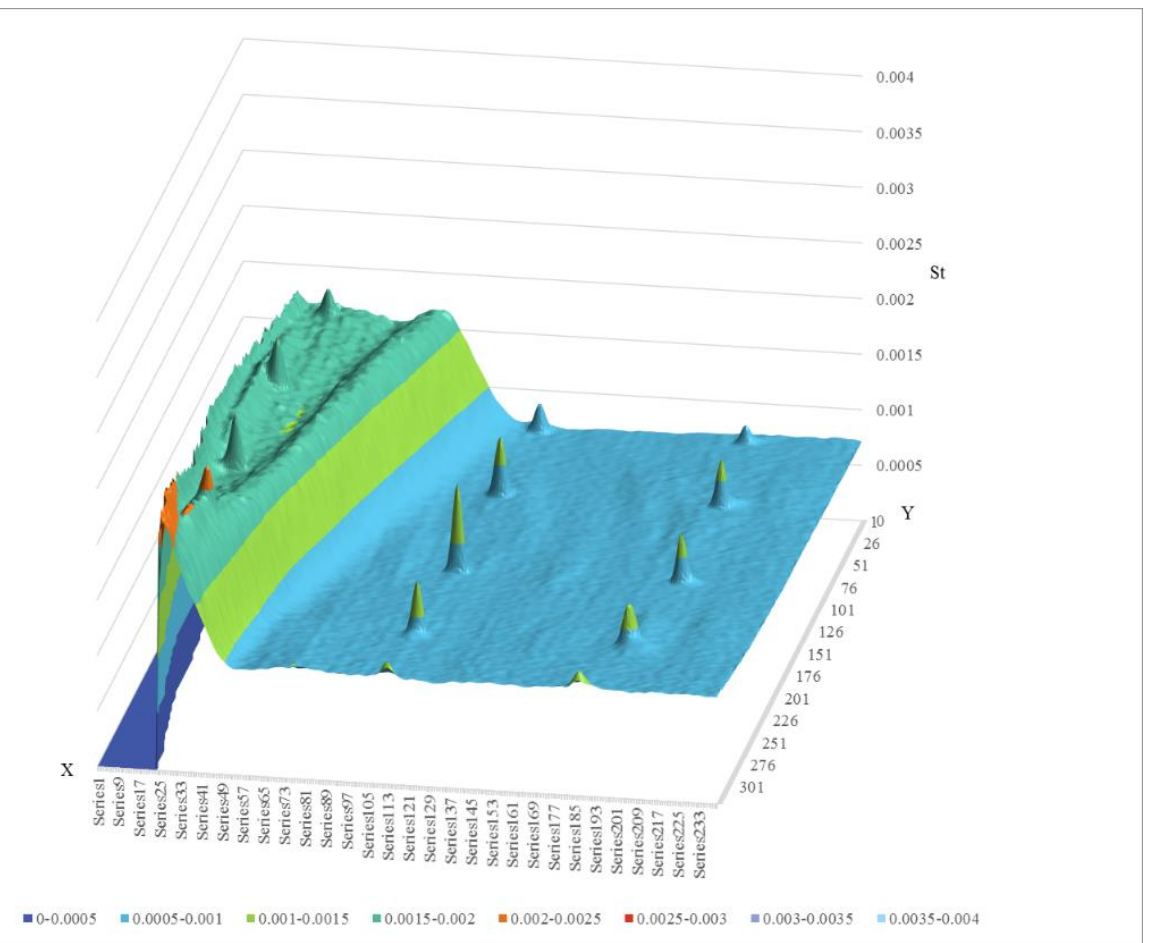

Figure 69. Suction surface Stanton number chart $\mathrm{Re}=1,000,000, \mathrm{Tu}=0.035$, heated starting length, bottom

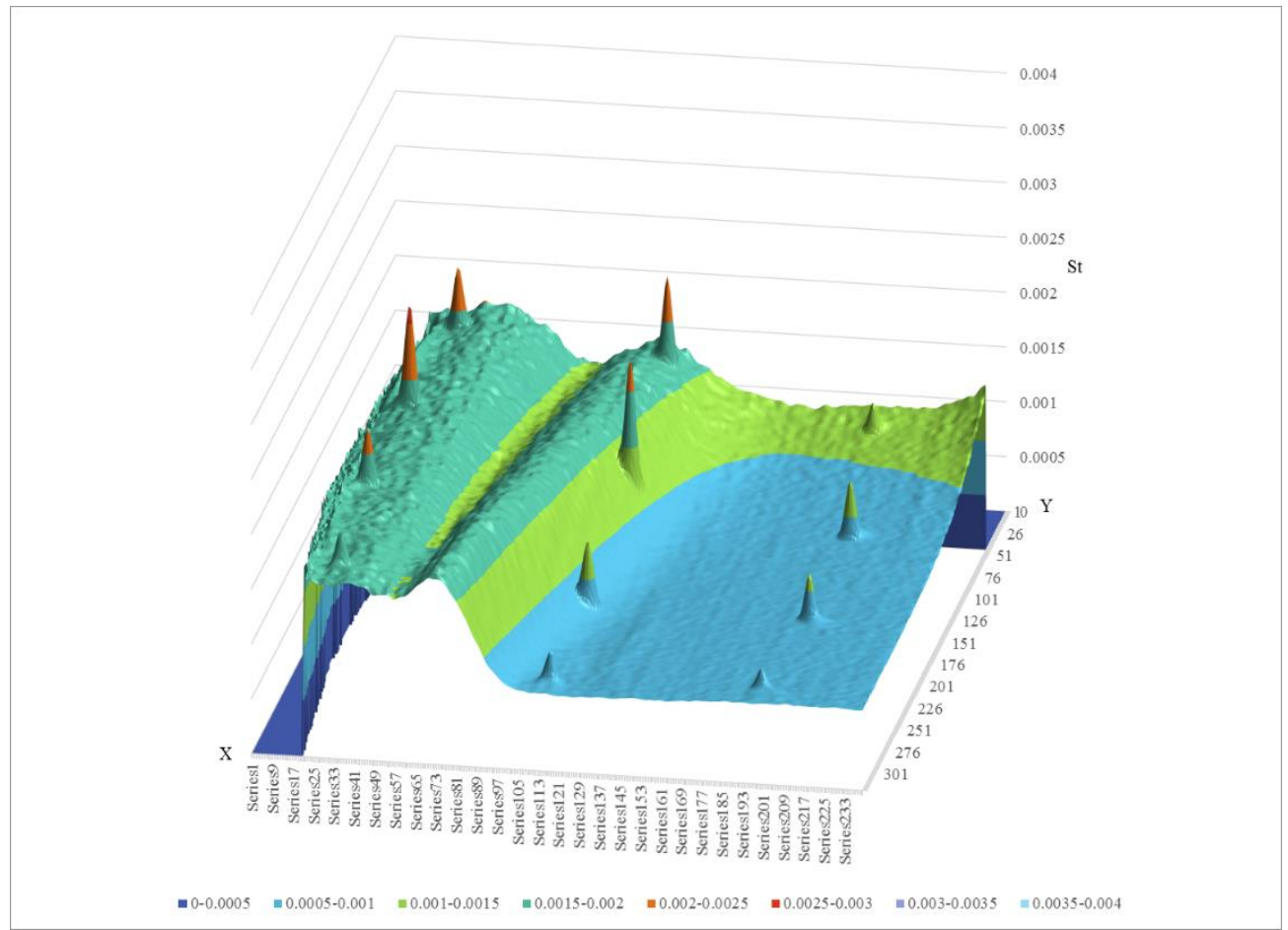

Figure 70. Suction surface Stanton number chart $\mathrm{Re}=1,000,000, \mathrm{Tu}=0.035$, heated starting length, top 


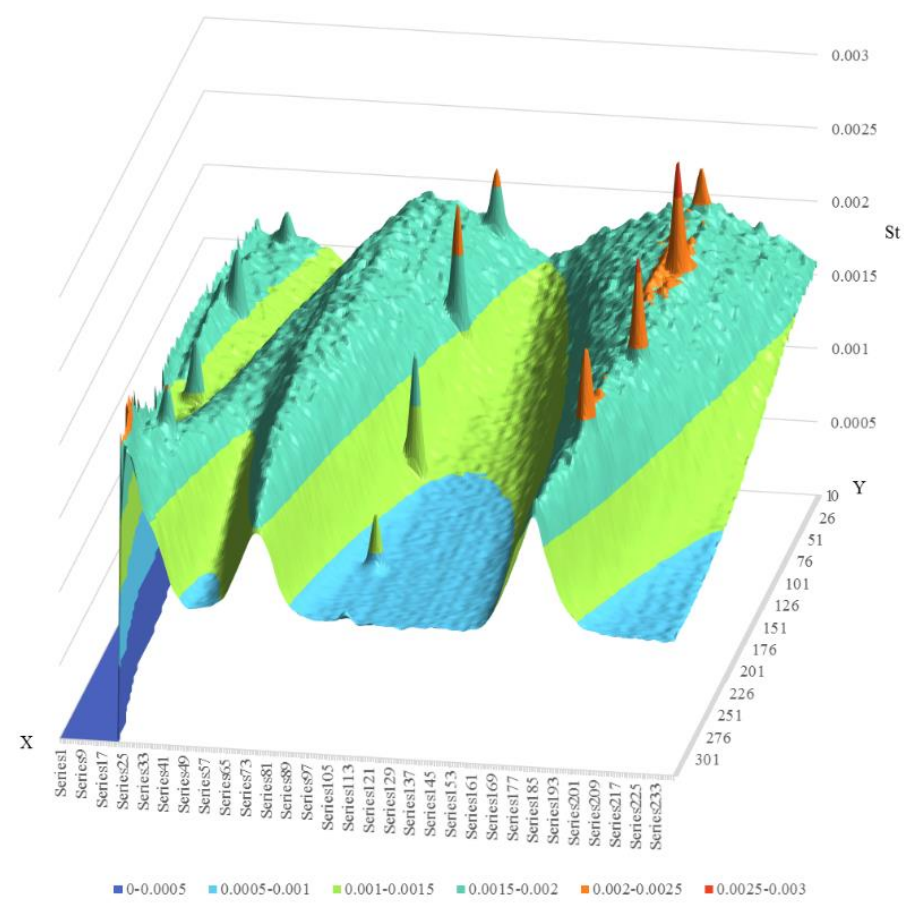

Figure 71. Suction surface Stanton number chart $\mathrm{Re}=2,000,000, \mathrm{Tu}=0.035$, heated starting length, bottom

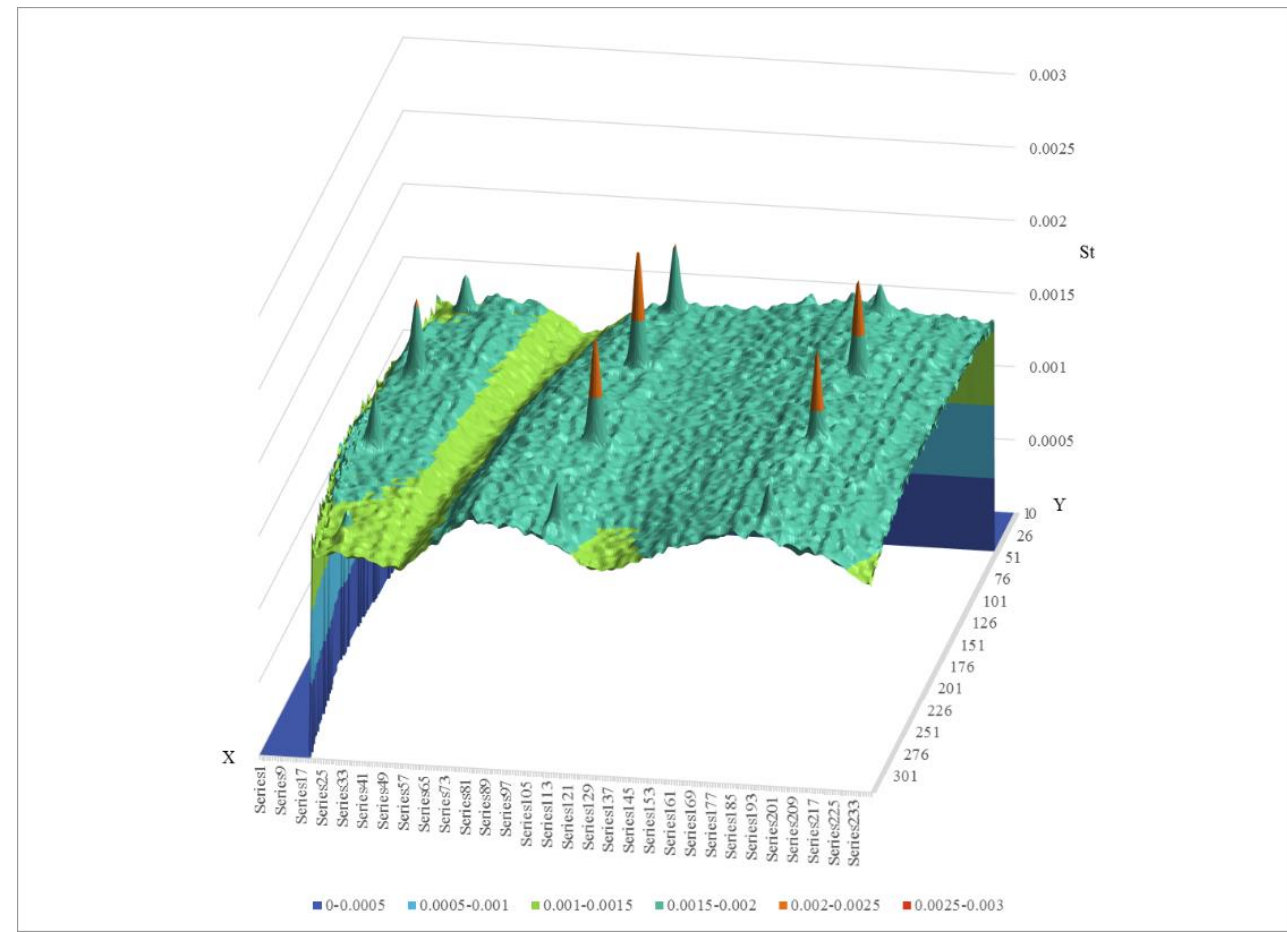

Figure 72. Suction surface Stanton number chart $\mathrm{Re}=2,000,000, \mathrm{Tu}=0.035$, heated starting length, top 


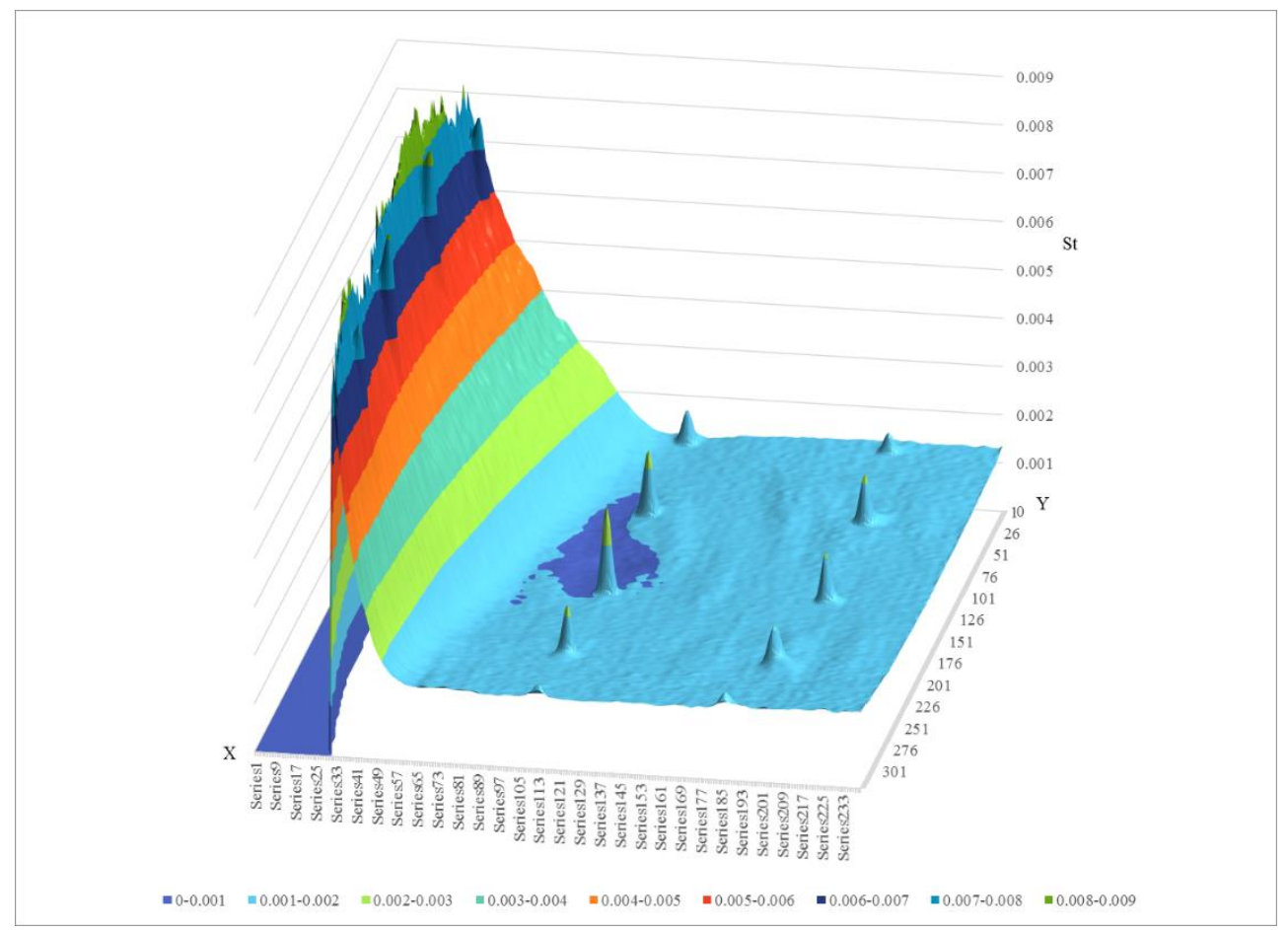

Figure 73. Suction surface Stanton number chart $\mathrm{Re}=500,000, \mathrm{Tu}=0.081$, unheated starting length, bottom

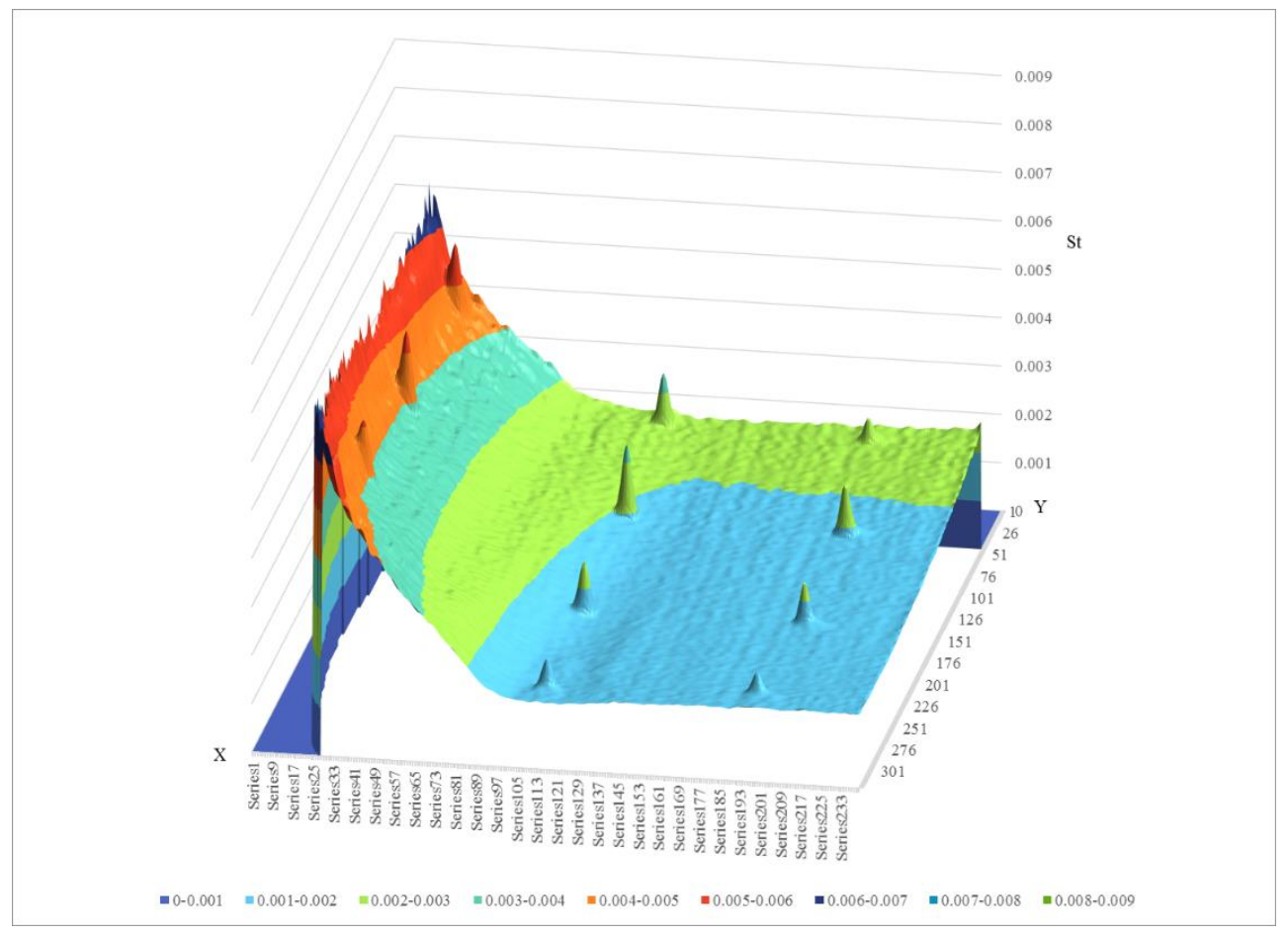

Figure 74. Suction surface Stanton number chart $\mathrm{Re}=500,000, \mathrm{Tu}=0.081$, unheated starting length, top 


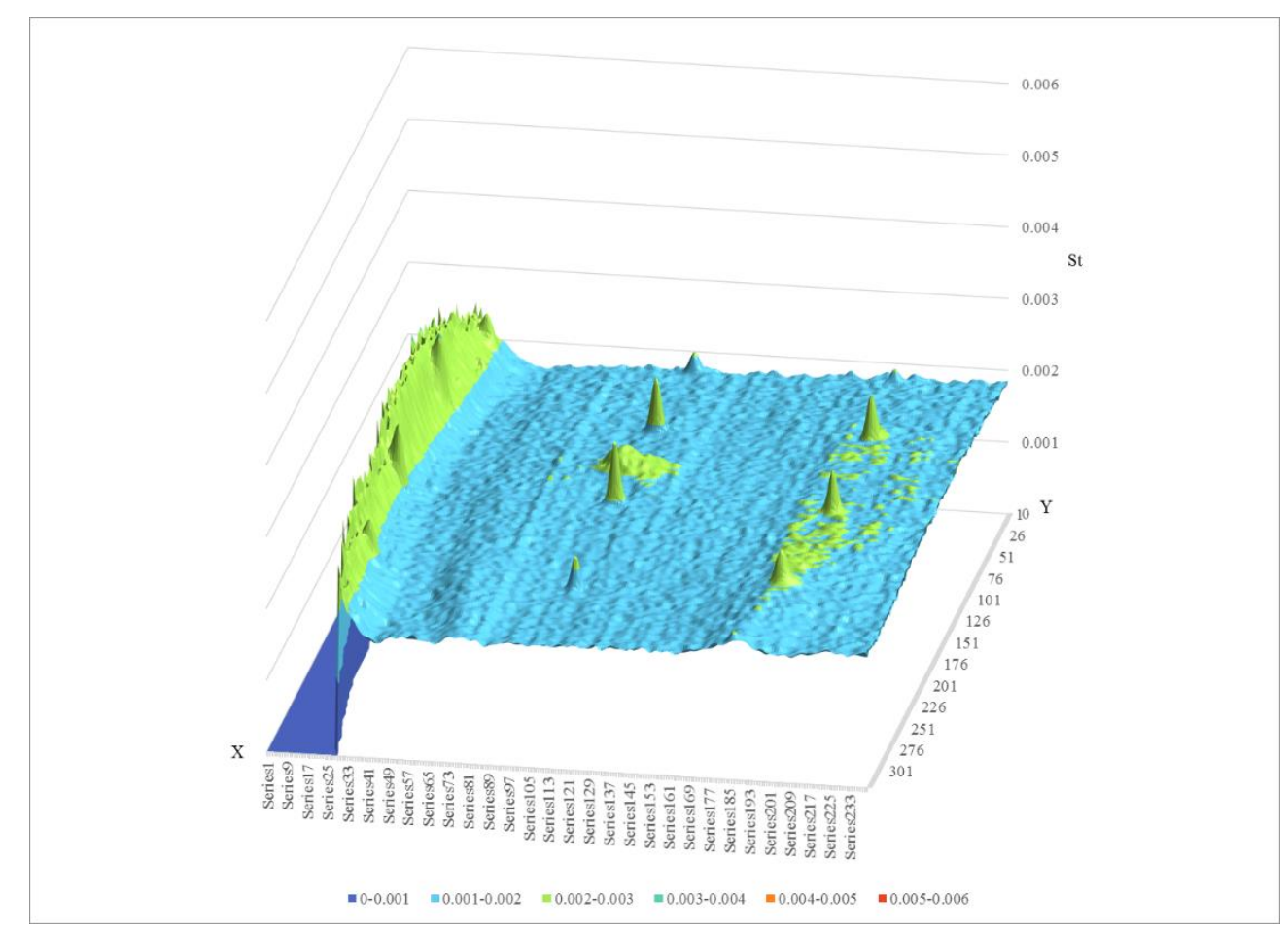

Figure 75. Suction surface Stanton number chart $\mathrm{Re}=2,000,000, \mathrm{Tu}=0.081$, unheated starting length, bottom

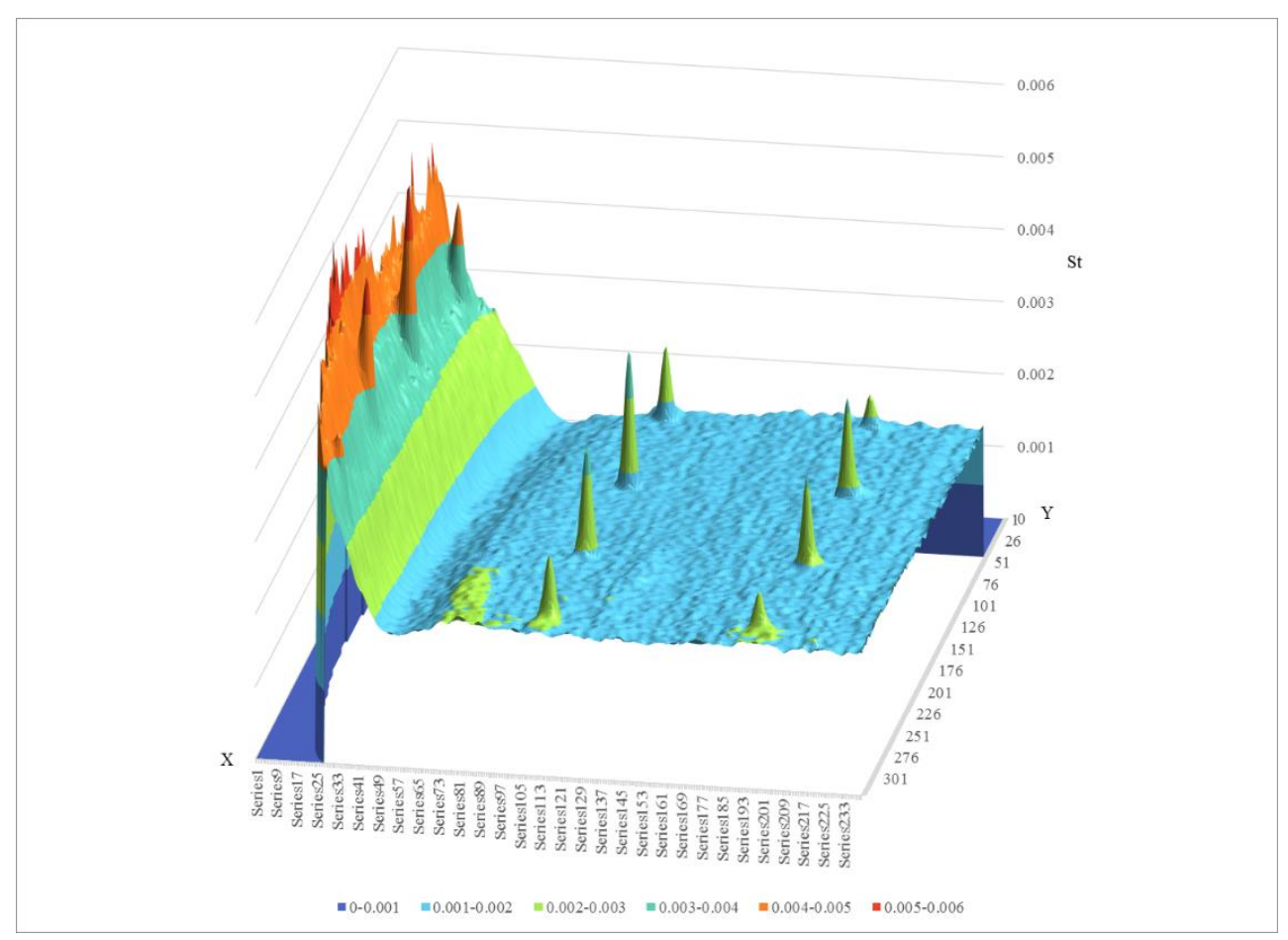

Figure 76. Suction surface Stanton number chart $\mathrm{Re}=2,000,000, \mathrm{Tu}=0.081$, unheated starting length, top 


\section{APPENDIX E \\ THE EFFECTS OF ROUGHNESS ON VANE HEAT TRANSFER}

This appendix should show the effects of the painted heat transfer vane compared to the standard heat transfer vane. It should be noted that the effects of roughness start to show itself in the form of early transition at a Reynolds number of 1,000,000 and continue to show themselves at a Reynolds number of 2,000,000. The visual effects can be seen in the suction surface heat transfer charts. The lower Reynolds number of 500,000 shows no signs of roughness affecting the heat transfer levels of the vane. Similar trends are seen for all turbulence levels.

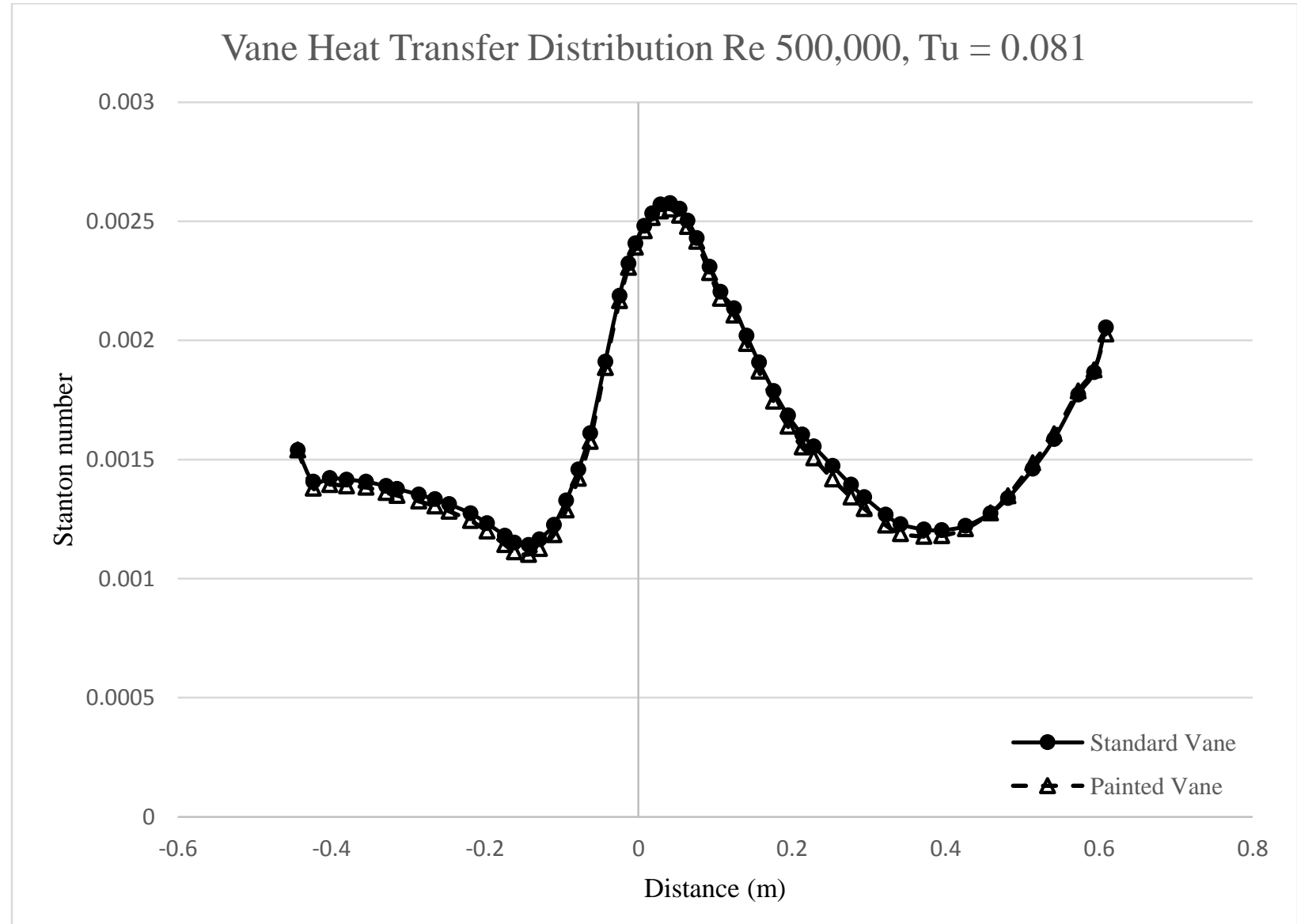

Figure 77. Vane heat transfer of the standard heat transfer vane vs. the painted heat transfer vane 


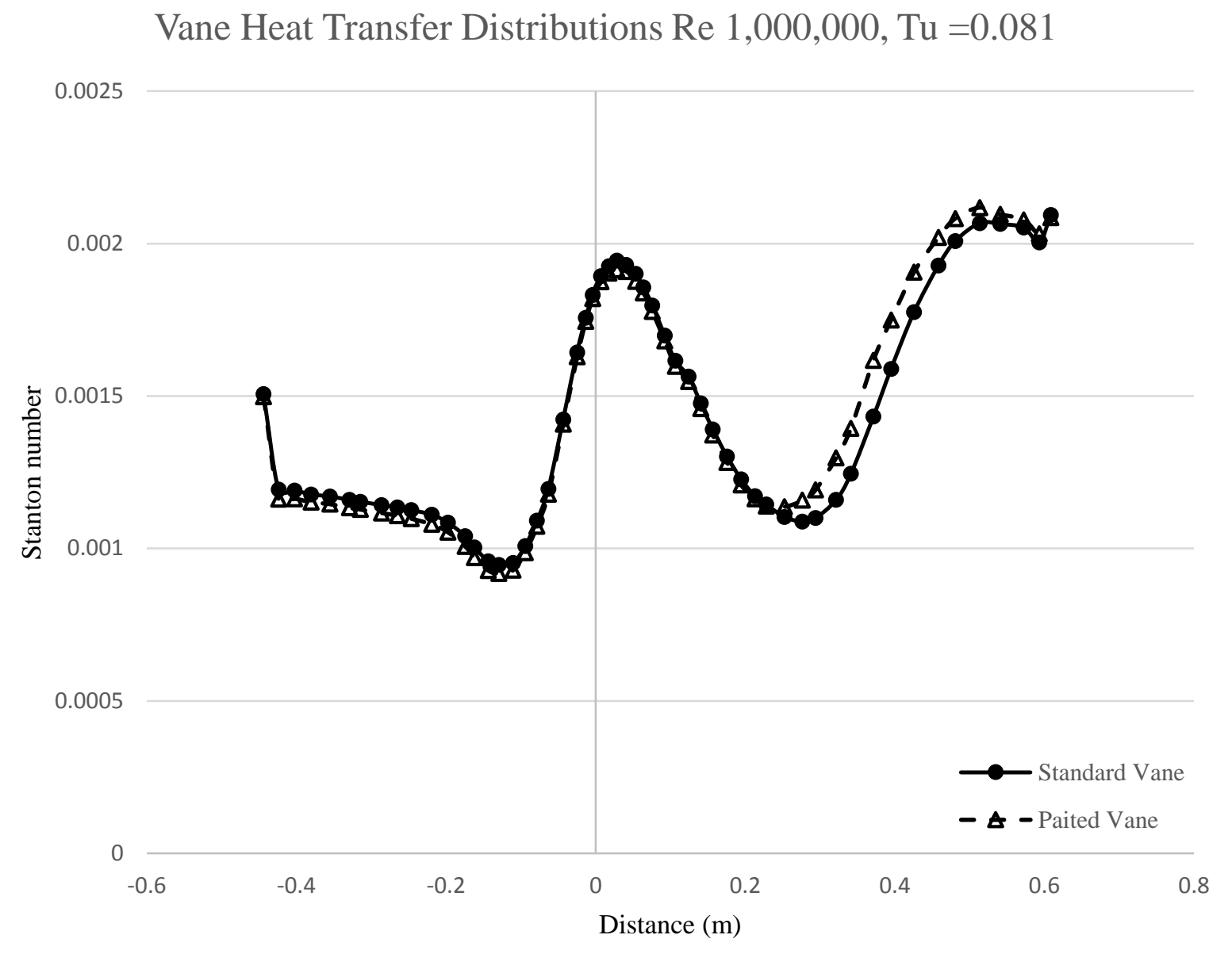

Figure 78. Vane heat transfer of the standard heat transfer vane vs. the painted heat transfer vane 


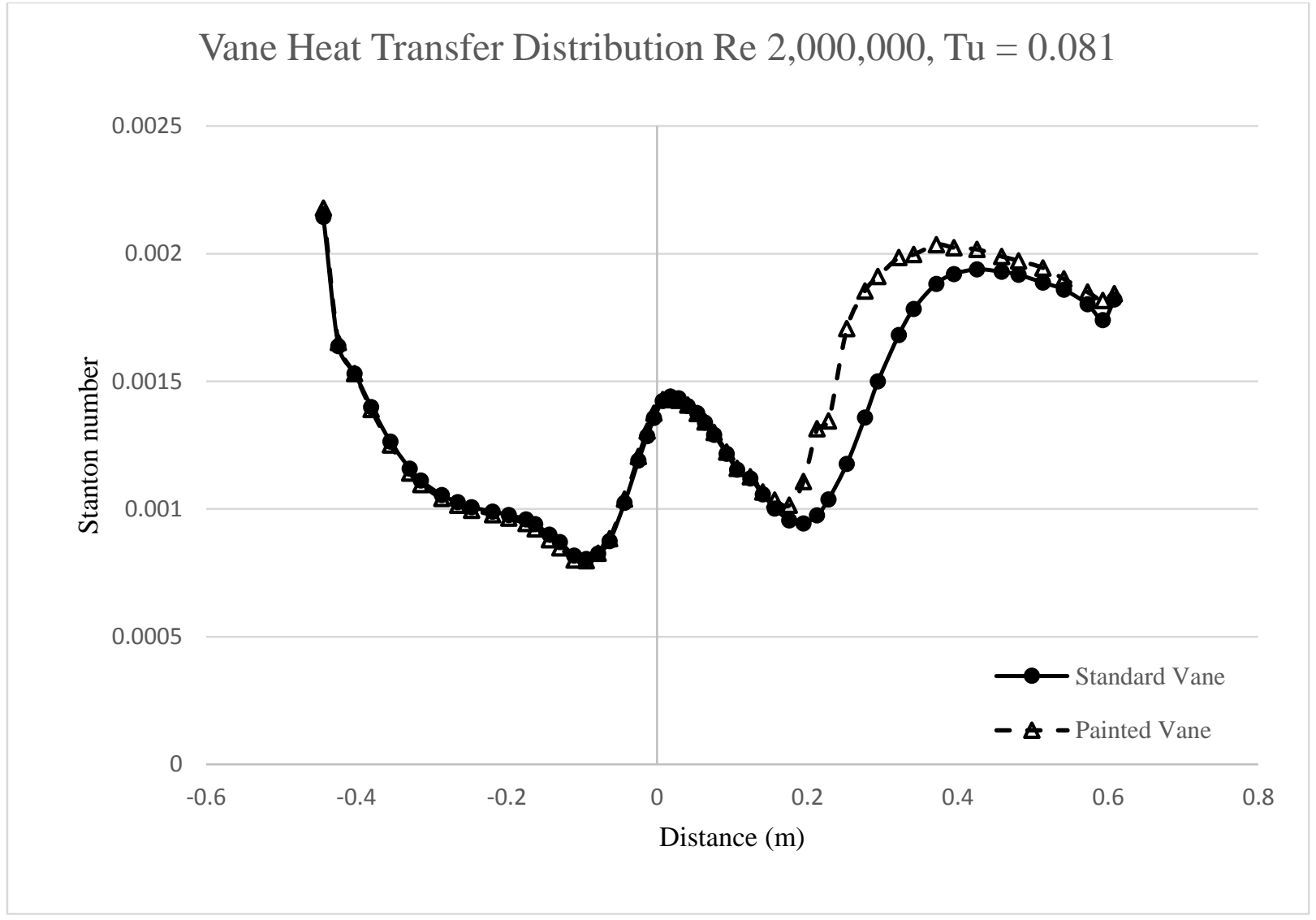

Figure 79. Vane heat transfer of the standard heat transfer vane vs. the painted heat transfer vane 


\section{APPENDIX F \\ UNCERTAINTY ANALYSIS}

The uncertainty analysis for this study was done using the root sum square method shown by Moffat [41]. These uncertainties arise from many different sources of error ranging from the data acquisition equipment, the fabrication of the parts, or procedure. All uncertainties were calculated with a $95 \%$ confidence interval.

The uncertainty in reporting the Stanton number for this study was calculated at a Reynolds number of $1,000,000$ at the varying turbulence conditions to see if they had any effect on the calculations. The uncertainty for the reported Stanton number is $+/-5.9 \%$. This value remains fairly constant for all turbulence levels, so it is fair to say that all calculations done on the exit Stanton number are consistent with each other. The largest uncertainty in the Stanton number is due to the local heat flux of the foil, which can mainly be attributed to the manufacturing process of the foil. Other area where there was uncertainty in the calculation was in the acquisition of the temperatures, both heated vane temperatures and adiabatic reference temperatures, the measured voltage across the foil, the area of the foil, the emissivity of the foil, the measured density of the air, and the measured exit velocity of the air. 


\section{REFERENCES}

[1] Van Fossen, G.J. and Bunker R.S., 2001, "Augmentation of stagnation region heat transfer due to turbulence from a DLN can combustor," ASME J. Turbomachinery, vol. 123, pp. 140146.

[2] Teekaram, A. J. H, Forth, C. J. P., and Jones, T. V., 1991, "Film cooling in the presence of mainstream pressure gradients," J. Turbomach., v. 113, p. 484.

[3] Busche, M.L., Kingery, J.E., and Ames, F.E., 2014, "Slot film cooling in an accelerating boundary layer with high free-stream turbulence,” ASME Paper No. GT2014-25360.

[4] Kingery, J., Ames, F., "Full Coverage Shaped Hole Film Cooling in an Accelerating Boundary Layer with High Free-Stream Turbulence," ASME. J. Turbomach, 138 (7), 071002 (Feb 17, 2016) (12 pages), doi10.1115/1.4031867.

[5] Zimmerman, D. R., 1979, "Laser anemometer measurements at the exit of a T63-C20 combustor," NASA CR-159623.

[6] Moss, R.W., and Oldfield, M.L.G., 1991, "Measurements of Hot Combustor Turbulence Spectra," ASME Paper No. 91-GT-351.

[7] Goebel, S. B., Abuaf, W., Lovette, T. A., and Lee, C.-P., "Measurement of Combustor Velocity and Turbulence Profiles," ASME Paper 93-GT-228.

[8] Zhang, L.J., and Gleazer, B., 1995, "Indirect turbulence measurement in gas turbine stages using heat flux probe," ASME Paper No. 95-GT-153.

[9] Zapp, G.M., 1950, "The effect of turbulence on local heat transfer coefficients around a cylinder normal to an air stream," Master's Thesis, Oregon State College.

[10] Chowdhury, N.H.K. and Ames, F.E., 2013, "The response of high intensity turbulence in the presence of large stagnation regions," ASME Paper No. GT2013-95055.

[11] Kingery, J.A., Ames, F.E., 2015, "Stagnation Region Heat Transfer Augmentation At Very High Turbulence Levels," ASME. J. Turbomach, 138 (8), 081005 (Mar 22, 2016) (10 pages), doi: 10.1115/1.4032677.

[12] Gandaparavu, P., and Ames, F.E., 2012 "The Influence of Leading Edge Diameter on Stagnation Region Heat Transfer Augmentation Including Effects of Turbulence Level, Scale, and Reynolds Number," ASME Journal of Turbomachinery, v. 135, pp. 011008-1-8 
[13] Van Fossen, G.J., Simoneau, R.J., and Ching, C.Y., 1995, "Influence of turbulence parameters, Reynolds number, and body shape on stagnation region heat transfer, ASME J. Heat Transfer, vol. 117, pp. 597-603.

[14] Dullenkopf, K., and Mayle, R.E., 1995, “An account of free-stream turbulence length scale on laminar heat transfer,” ASME J. Turbomachinery, vol. 117, pp. 401-406.

[15] Ames, F.E., and Moffat, R.J., 1990, "Heat Transfer with High Intensity, Large Scale Turbulence: The Flat Plate Turbulent Boundary Layer and the Cylindrical Stagnation Point," HMT-44 (Ph.D. Dissertation), Stanford University.

[16] Sanitjai, S. and Goldstein, R.J., 2001, "Effect of free stream turbulence on local mass transfer from a circular cylinder", International Journal of Heat and Mass Transfer, vol. 44, no. 15, pp. 2863-2875.

[17] Nix, A.C., Diller, T.E., and Ng, W. F., 2007, "Experimental Measurements and Modeling of the Effects of Large-Scale Freestream Turbulence on Heat Transfer," ASME J. Turbomach., vol. 129 , pp. 542-550.

[18] Nix, A.C., Diller, T.E., 2005, "Experiments on the Physical Mechanism of Heat Transfer Augmentation by Freestream Turbulence at a Cylinder Stagnation Point, GT-2005-68616.

[19] Gifford, A.R., Diller, T.E., and Vlachos, P.P., 2011, "The Physical Mechanism of Heat Transfer Augmentation in Stagnation Flow Subject to Freestream Turbulence," ASME J. Heat Transfer, vol. 133, 021901-1-11.

[20] Boyle, R.J., and Russell, L.M., 1989, "Experimental Determination of Stator Endwall Heat Transfer," ASME Paper No. 89-GT-219

[21] Harvey, N.W., and Jones, T.V., 1990, "Measurement and Calculation of Endwall Heat Transfer and Aerodynamics on a Nozzle Guide Vane in Annular Cascade," ASME Paper No. 90-GT-301

[22] Spencer, M. C., Jones, T. V., and Lock, G. D., 1996, “Endwall Heat Transfer Measurements in an Annular Cascade of Nozzle Guide Vanes at Engine Representative Reynolds and Mach Numbers," International Journal of Heat Fluid Flow, vol. 17, pp. $139-147$.

[23] Kumar, G. N., R. M. Jenkins, and U. Sahu. "Regionally Averaged Endwall Heat Transfer Correlations for a Linear Vane Cascade." Volume 3: Heat Transfer; Electric Power (1985)

[24] Radomsky, R.W., and Thole, K.A., 2000, "High Free-Stream Turbulence Effects on Endwall Heat Transfer for a Gas Turbine Stator Vane,” Journal of Turbomachinery, vol. 122, pp. 699-708 
[25] Ames, F.E., Barbot, P.A., Wang, C., 2002, "Effects of Aeroderivative Combustor Turbulence on Endwall Heat Transfer Distributions Acquired in a Linear Vane Cascade," ASME Paper No. GT-2002-30525, Accepted for Publication in ASME Journal of Turbomachinery.

[26] Ames, F.E., Barbot, P.A., Wang, C., "Effects of Catalytic and Dry Low NOx Combustor Turbulence on Endwall Heat Transfer Distributions," Abstract No. GT-200338507, offered for presentation at the 2003 Turbo Expo, Atlanta, Georgia.

[27] Hylton, L.D., Mihelc, M.S., Turner, E.R., Nealy, D.A., and Your, R.E., 1983, “Analytical and Experimental Evaluation of the Heat Transfer Distribution Over the Surface of Turbine Vanes," NASA CR-168015.

[28] Arts, T., Lambert de Rouvroit, M., and Rutherford, A.W., 1990, "Aero-thermal Investigation of a Highly Loaded Transonic Linear Turbine Guide Vane Cascade," Technical Note 174, von Karman Institute for Fluid dynamics, Belgium.

[29] Azad, Gm S., Je-Chin Han, Shuye Teng, and Robert J. Boyle. "Heat Transfer and Pressure Distributions on a Gas Turbine Blade Tip." Volume 3: Heat Transfer; Electric Power; Industrial and Cogeneration (2000)

[30] Radomsky, R. and Thole, K.A., 2000, "Highly Turbulent Flowfield Measurements Around a Stator Vane," ASME Journal of Turbomachinery, Vol. 122, pp. 255-262.

[31] Ames, F.E., 1997, "The Influence of Large Scale, High Intensity Turbulence on Vane Heat Transfer," ASME Journal of Turbomachinery, vol. 119, pp. 23.

[32] Ames, F.E., Wang, C., and Barbot, P.A., 2003, "Measurement and prediction of the influence of catalytic and dry low NOx Combustor Turbulence on Vane Surface Heat Transfer," ASME Journal of Turbomachinery, vol. 125, pp. 210-220.

[33] Ames, F.E., Argenziano, M., Wang, C., 2004, "Measurement and Prediction of Heat Transfer Distributions on an Aft Loaded Vane Subjected to the Influence of Catalytic and Dry Low NOx Combustor Turbulence," ASME Journal of Turbomachinery, vol 126, pp. 139-149.

[34] ANSYS Fluent Release 16.0 Copyright 2014, ANSYS, Inc..

[35] Kays, W.M., 1987, "STAN7, a finite difference boundary layer code.”

[36] Ames, F.E., Kwon, O., and Moffat, R.J., 1999, “An algebraic model for high intensity large scale turbulence," ASME Paper No. 99-GT-160.

[37] Mayle, R.E., 1991, "The Role of Laminar-Turbulent Transition in Gas Turbine Engines," ASME Journal of Turbomachinery, Vol. 113, pp. 509-537.[38] T.-H. Shih, W. W. Liou, A. Shabbir, and J. Zhu, 1995, "A New k- $\varepsilon$ Eddy-Viscosity Model for High Reynolds Number Turbulent Flows - Model Development and Validation," Computers Fluids, 24(3), pp. 227-238. 
[38] Kays, W.M., Crawford, M.E, and Weigand, B.,, 2005, Convective Heat and Mass Transfer, $4^{\text {th }}$ Ed., McGraw-Hill, NY.

[39] Sieverding C. H., 1984, "Recent Progress in the Understanding of Basic Aspects of Secondary Flows in Turbine Blade Passages," Journal of Engineering for Gas Turbines and Power, Vol. 107, pp. 248-257

[40] Langston, L.S., Nice, M.L., and Hooper, R.M., 1977, “Three-dimensional flow within a turbine cascade passage,” ASME Journal of Engineering for Power, January, 1977, pp. 21-28

[41] Moffat, R.J., 1988, "Describing the Uncertainties in Experimental Results," Experimental Thermal and Fluid Science, v. 1, pp. 3-17.

[42] Kingery, J.E., 2015, “An Experimental Investigation into the Effects of High Free-Stream Turbulence on full Coverage Shaped Hole Film Cooling in an Accelerating Boundary Layer," University of North Dakota, Grand Forks, ND.

[43] Barbot, P.A., 2003, "The Effects of Combustor Generated Turbulence and Reynolds Number on Endwall Heat Transfer Distributions in a Linear Vane Cascade," University of North Dakota, Grand Forks, ND.

[44] Jaswal, I., 2008, “Aerodynamic Losses and Heat Transfer for a Covered Trailing Edge Turbine Bane with a High Solidity Low Pressure Drop Pin Fin Array and Variable Coolant Ejection," University of North Dakota, Grand Forks, ND. 